University of Nebraska - Lincoln

DigitalCommons@University of Nebraska - Lincoln

Anthony F. Starace Publications

Research Papers in Physics and Astronomy

August 1984

\title{
Effects of Magnetic and Electric Fields on Highly Excited Atoms
}

Charles W. Clark

SERC Daresbury Laboratory, England

K. T. Lu

Argonne National Laboratory, Argonne, IL

Anthony F. Starace

University of Nebraska-Lincoln, astarace1@unl.edu

Follow this and additional works at: https://digitalcommons.unl.edu/physicsstarace

Part of the Physics Commons

Clark, Charles W.; Lu, K. T.; and Starace, Anthony F., "Effects of Magnetic and Electric Fields on Highly Excited Atoms" (1984). Anthony F. Starace Publications. 152.

https://digitalcommons.unl.edu/physicsstarace/152

This Article is brought to you for free and open access by the Research Papers in Physics and Astronomy at DigitalCommons@University of Nebraska - Lincoln. It has been accepted for inclusion in Anthony F. Starace Publications by an authorized administrator of DigitalCommons@University of Nebraska - Lincoln. 
Published in PROGRESS IN ATOMIC SPECTROSCOPY, Part C.

Edited by H. J. Beyer and Hans Kleinpoppen.

(New York, Plenum Publishing Corp., 1984)

This article, co-authored by a U.S. government employee, is not subject to copyright.

\title{
7 \\ Effects of Magnetic and Electric Fields on Highly Excited Atoms
}

\author{
Charles W. Clark, K. T. Lu, and Anthony F. Starace
}

\section{Introduction}

The long and glittering history of the study of atoms in external electric and magnetic fields dates from the late 19th century work of Zeeman ${ }^{(1)}$ and the early 20th century works of Stark ${ }^{(2)}$ and Paschen and Back. ${ }^{(3)}$ The birth of quantum mechanics was followed by the pioneering studies of diamagnetic effects of Van Vleck ${ }^{(4)}$ and Jenkins and Segré, ${ }^{(5)}$ and by the prediction of Landau resonances ${ }^{(6)}$ in free-electron spectra of solids. More recently, observations of highly excited atoms in external fields, ${ }^{(7-10)}$ made possible by the advent of high-resolution spectrometers, superconducting magnets, and lasers, have led to a revitalization of atomic spectroscopy. In particular, the observation of field-induced resonances in an otherwise smooth continuum ${ }^{(11-13)}$ has compelled theory ${ }^{(14-16)}$ to deal with a class of phenomena involving competing forces of different symmetries and comparable magnitudes. Fragmentary advances of theoretical understanding have in turn pointed to possibilities of using external fields as probes of atomic structure. In this sense the present lines of investigation adhere to

Charles W. Clark - SERC Daresbury Laboratory, Daresbury, Warrington WA44AD, England. K. T. LU • Argonne National Laboratory, Argonne, Illinois 60439. Anthony F. STARACE - Behlen Laboratory of Physics, The University of Nebraska, Lincoln, Nebraska 68588-0111. Work supported by Office of Basic Energy Sciences, Division of Chemical Sciences, U.S. Department of Energy, under contract No. W-31-109-Eng-38; also, for Dr. Starace's work, under contract No. EY-76-S-02-2892. Present address for Dr. Clark: Atomic and Plasma Radiation Division, National Bureau of Standards, Washington, D.C. 
the spirit of Professor Hanle's pioneering work, and we are honored to be able to dedicate this article to him on the occasion of his eightieth birthday.

This review describes primarily recent theoretical developments on highly excited atoms in uniform external fields, and complements the experimental review of Gay in the present volume. The Zeeman and Stark effects on low-lying atomic states, on the other hand, constitute a mature field of study which has been reviewed previously by Garstang, ${ }^{(17)}$ Kollath and Standage, ${ }^{(18)}$ Bayfield, ${ }^{(19)}$ and Kleppner. ${ }^{(20)}$ Most theoretical work on highly excited states in laboratory strength fields has heretofore focused on the prototype system of atomic hydrogen, and accordingly hydrogen receives special emphasis in this article. For nonhydrogenic atoms we review theoretical work using the framework of quantum defect theory. Magnetic and electric effects are treated separately and in combination. For magnetic fields of astrophysical magnitude, on the other hand, the competition between external and atomic forces becomes important for low-lying states. Recent developments in the understanding of hydrogen in such fields are reviewed here, and qualitative aspects of general atomic structure in such fields are briefly discussed.

As indicated above, the principal difficulty in dealing with highly excited atoms in external fields arises from the simultaneous presence of separate strong forces of different symmetry. For hydrogen in a magnetic field, the two forces involved are the spherically symmetric Coulomb interaction between the electron and the proton, and the cylindrically symmetric interaction between the electron and the magnetic field. Specifically, the potential consists of two terms: the Coulomb potential, $-1 / r$, and the diamagnetic potential, proportional to $r^{2} \sin ^{2} \theta$, where $\theta$ is the angle between the magnetic field axis and the electron position vector. These two in combination yield a Schrödinger equation which is nonseparable in any coordinate system. When one of the potentials is significantly smaller than the other, for instance at small $r$ where the Coulomb potential is dominant, the problem can be solved by perturbation theory. This is the case for low-lying states in laboratory magnetic fields. For highly excited states, however, as the principal quantum number $n$ increases, the electron moves to larger distances $r$ where the strength of the diamagnetic potential becomes comparable to the Coulomb binding. As a result, one observes that as $n$ increases there is a transition from a primarily Rydberg-like spectrum, through a region in which there is a breakdown of the zero-field Rydberg classification of states, to a new spectral regime of quasi-Landau resonances. This is also the case for nonhydrogenic atoms, but the spectrum of hydrogen is distinctive because of the zero field degeneracy of levels. We consider recent classical, semiclassical, and quantum mechanical approaches to the understanding of the spectrum. A partial classification of the hydrogen spectrum in the transition region is discussed. 
The same competition between external and atomic forces occurs for highly excited states of hydrogen in a uniform electric field. However, the Schrödinger equation is separable in parabolic coordinates. Solutions to the separated equations are not available in closed form, so that approximate analytical or numerical means must be employed. For nonhydrogenic atoms, this separability of the equations of motion is broken near the ionic core. Thus again, although the dynamics of electron motion at large distances are the same for all atoms, the problem of hydrogen is unique. We discuss recent developments in analytical and numerical approaches to its solution.

The differences between hydrogen and other atoms arise from the presence of a non-Coulombic interaction between the excited electron and the residual ion core. In the absence of external fields, the effects of this interaction are conveniently characterized by quantum defects. ${ }^{(21-24)}$ When external fields are present, quantum defects may still be used to describe this short-range interaction, because the magnitude of the external fields is negligible in comparison to the internal atomic fields. Thus we employ quantum defect theory as a tool for analyzing nonhydrogenic spectra in external fields. If one knows the analytic solutions of the Schrödinger equation in the region outside the core where only the net Coulomb and external field potential(s) are present, then the quantum defects may be used to construct a linear combination of these solutions that represents the electron wave function everywhere outside the core. For the diamagnetic problem such analytic solutions are not yet available, and so the present utility of a quantum defect approach is essentially restricted to perturbative treatments, which we shall review here. For the Stark effect, on the other hand, such solutions can be obtained in parabolic coordinates. Since, however, the usual quantum defect theory requires knowledge of the wave function on a spherical boundary enclosing the core, the matching of parabolic coordinate solutions to spherical boundary conditions is nontrivial. The solution to this matching problem has recently been obtained, and we shall review its main features.

As well as reviewing the Zeeman and Stark effects as distinct phenomena, we shall also treat cases in which both fields are simultaneously present. For the magnetic problem such a study is necessary because the center-ofmass motion of the atom induces an electric field in the atomic rest frame. Such motional Stark fields have been observed to have pronounced effects on the spectra of light atoms. We discuss present theoretical understanding of this phenomenon. This motional Stark effect is a special case of the general problem of crossed magnetic and electric fields, whose principal features we also review.

For laboratory strength fields the competition between external and atomic forces becomes significant only in highly excited states. For very 
high fields, such as may occur in astrophysics, this competition is important for low-lying excited states and even the ground states of atoms. In fact, for magnetic fields of the magnitude expected to exist on the surfaces of neutron stars, the magnetic field dominates the electronic motion in such a way as to radically change atomic structure. We shall review some of the general properties of atoms in such fields.

\section{Diamagnetic Effects in the Hydrogen Atom}

Effective use of a general quantum defect formulation can only be made if one has some knowledge of the solutions of the equations of motion for the electron in the region outside the atomic core. When the electron wave function in the exterior region is adequately represented by a perturbed Coulomb wave function, matching it to boundary conditions imposed near the atomic core is straightforward. This will be shown in Section 4.4. below. However, when the magnetic and Coulomb potentials become comparable in strength, perturbation theory is unsatisfactory and a direct solution to the equations of motion must be sought. At present no general method for obtaining such solutions is known. Thus most previous theoretical effort has been directed towards identifying and elaborating qualitative properties of the electron motion in the exterior region, and, recently, towards obtaining accurate wave functions for hydrogenic atoms. In this section we will review the development of the theory along these lines. Some progress towards a general theory is evident, but it has not reached the stage where a definitive treatment is possible.

\subsection{The Equations of Motion}

We consider first the classical Lorentz equation for the motion of an electron and a proton in a magnetic field:

$$
\begin{aligned}
& m_{e} \ddot{\mathbf{r}}_{e}=-\frac{e^{2} \mathbf{r}}{r^{3}}-\frac{e}{c} \dot{\mathbf{r}}_{e} \times \mathbf{B} \\
& m_{p} \ddot{\mathbf{r}}_{p}=\frac{e^{2} \mathbf{r}}{r^{3}}+\frac{e}{c} \dot{\mathbf{r}}_{p} \times \mathbf{B}
\end{aligned}
$$

where $\mathbf{r}=\mathbf{r}_{e}-\mathbf{r}_{p}$. If we take $M=m_{e}+m_{p}, \mathbf{R}=\left(m_{p} \mathbf{r}_{p}+m_{e} \mathbf{r}_{e}\right) / M$, and $\mu=$ $m_{p} m_{e} /\left(m_{e}+m_{p}\right)$ as is usually done to separate center-of-mass and internal motions, we find that

$$
M \dot{\mathbf{R}}+\frac{e}{c} \mathbf{r} \times B=\pi
$$


where $\pi$ is a constant. Thus $\pi$ is to be identified with the net momentum of the system; the kinetic momentum $M \dot{\mathbf{R}}$ is not constant in time, but is coupled to the electric dipole moment $-e \mathbf{r}$ of the system. By combination of (1) and (2) the equation of motion for $\mathbf{r}$ is readily shown to be

$$
\mu \ddot{\mathbf{r}}=-\frac{e^{2} \mathbf{r}}{r^{3}}-\frac{e}{c}\left(\sigma \dot{\mathbf{r}}-\frac{e}{M c} \mathbf{r} \times \mathbf{B}\right) \times \mathbf{B}-\frac{e}{M c} \boldsymbol{\pi} \times \mathbf{B}
$$

where $\sigma=\left(m_{p}-m_{e}\right) / M \simeq 1$. The rightmost term of (3) indicates the presence of a uniform electric field in the center-of-mass frame, induced by the motion of that frame across the magnetic field lines. In the remainder of this section we shall take $\pi \times \mathbf{B}=0$. The effect of the motional electric field in the general case is discussed in Section 5.2 below. The second rightmost term may be simplified by writing the equations of motion in a coordinate frame rotating about the magnetic field axis: the angular frequency of rotation being either of $\omega_{ \pm}=(e B / \mu c)(\sigma \pm 1) / 2$, corresponding, respectively, to a counterclockwise rotation at approximately the electron cyclotron frequency $e B / m_{e} c$ or a clockwise rotation at approximately the proton cyclotron frequency $e B / m_{p} c$. Either choice gives equivalent results, but since $\left|\omega_{-}\right| \ll\left|\omega_{+}\right|$we shall express the equations of motion in the slowly rotating frame. They are

$$
\mu \ddot{\mathbf{r}}=-\frac{e^{2} \mathbf{r}}{r^{3}}-\frac{e}{c} \mathbf{r} \times \mathbf{B}
$$

Thus the classical equations of motion in the rotating frame are equivalent to those for an electron of reduced mass $\mu$ moving in a fixed Coulomb potential in the presence of a magnetic field. We shall hereafter discuss just this idealized problem, its relation to realistic cases being taken as understood from the above arguments. In calculation of energies it must be remembered that the rotation of the coordinate frame produces a current which gives a slight paramagnetic energy; this appears in quantum mechanics as an adjustment of the electron's Landé $g$ factor.

The formal separation of the center-of-mass motion in quantum mechanics is straightforward. Finite nuclear mass corrections to the Hamiltonian for a hydrogen atom in a uniform magnetic field were derived to first order in the electron-proton mass ratio of Lamb. ${ }^{(25)}$ A physically intuitive derivation of these corrections has also been given by Bethe and Salpeter. ${ }^{(26)}$ Interest in exotic atoms such as muonium and positronium, which have much larger mass ratios, led $\mathrm{Carter}^{(27)}$ to solve the nuclear motion problem exactly for neutral two-body systems. Recently the problem has been reexamined by a number of authors. ${ }^{(28-34)}$ In particular, 
Avron et al. ${ }^{(30)}$ have examined the general question of the separability of center-of-mass motion for $N$ charged particles in a uniform magnetic field.

The Hamiltonian for atomic hydrogen in a uniform magnetic field $B$ directed along the $z$ axis is

$$
H=\sum_{i=1}^{2} \frac{\left[\mathbf{p}_{i}-q_{i} \mathbf{A}\left(\mathbf{r}_{i}\right)\right]^{2}}{2 m_{i}}-\frac{e^{2}}{\left|\mathbf{r}_{1}-\mathbf{r}_{2}\right|}
$$

where the index 1 refers to the electron and the index 2 refers to the proton, $-q_{1}=q_{2}=e$, and the vector potential is chosen in the Landau gauge, $A_{y}=B x, A_{x}=A_{z}=0$. Carter ${ }^{(27)}$ has shown that the total wave function described by the Hamiltonian in (5) may be written in terms of the center-of-mass coordinate $R=(X, Y, Z)$ and the relative coordinate $r=(x, y, z)$ as

$$
\Psi(\mathbf{R}, \mathbf{r})=\exp i(\mathbf{P} \cdot \mathbf{R}+e B y \boldsymbol{X}) \psi(\mathbf{r})
$$

where $\mathbf{P}$ is the center-of-mass momentum and $\psi(\mathbf{r})$ is described by the following reduced Hamiltonian in the relative coordinate $\mathbf{r}$ :

$$
h=\frac{1}{2 \mu}\left(\mathbf{p}_{\mu}+\frac{e}{c} \mathbf{a}\right)^{2}-\frac{e^{2}}{r}-\frac{e \mathbf{r}}{c} \cdot\left(\frac{\mathbf{P}}{M} \times \mathbf{B}\right)+\frac{P^{2}}{2 M}+\frac{e^{2} B^{2}}{2 M c^{2}}\left(x^{2}+y^{2}\right)
$$

In Eq. (7), $\mu$ is the reduced mass, $\mathbf{p}_{\mu}$ is the momentum operator for the reduced mass particle, and $\mathbf{a}$ is a new vector potential defined by

$$
\boldsymbol{\nabla} \times \mathbf{a}=\left(\frac{m_{2}-m_{1}}{m_{1}+m_{2}}\right) \mathbf{B}=\mathbf{b}
$$

The first two terms in (7) represent the usual Hamiltonian for a particle of mass $\mu$ moving in the Coulomb field and in the magnetic field given in (2.8); the third term represents the interaction of the reduced mass particle with the motional electric field $\mathbf{E}=(\mathbf{P} / M) \times \mathbf{B}$ arising from the center-ofmass velocity $(\mathbf{P} / \boldsymbol{M})$; the fourth term is the kinetic energy of center-of-mass motion; and the last term is a harmonic oscillator potential. As pointed out by O'Connell, ${ }^{(31)} \mathrm{Eq}$. (7) may be greatly simplified by means of a judicious choice of gauge for the vector potential a: $a_{x}=-b y / 2, a_{y}=b x / 2$, $a_{z}=0$. In this gauge and switching to the center-of-mass coordinate system (i.e., setting $\mathbf{P}=0$ ) one finds that $h$ reduces to

$$
h=\frac{p_{\mu}^{2}}{2 \mu}-\frac{e^{2}}{r}+\frac{e}{2 \mu c} g B L_{z}+\frac{e^{2}}{8 \mu c^{2}} B^{2}\left(x^{2}+y^{2}\right)
$$


where

$$
g \equiv \frac{m_{2}-m_{1}}{m_{1}+m_{2}}
$$

The reduced Hamiltonian $h$ in Eq. (9) is idential to that for a particle of mass $\mu$ moving in a Coulomb field as well as a uniform magnetic field $B$ except for the presence of the factor $g$ in the linear Zeeman term instead of the usual factor unity. Interestingly, $g / \mu=m_{1}^{-1}+m_{2}^{-1}$ is Lamb's ${ }^{(25)}$ correction for center-of-mass motion.

In summary, then, in the center-of-mass coordinate system the effect of a finite nuclear mass of the Hamiltonian for a hydrogen atom in a uniform magnetic field is to replace the electron mass by the reduced mass and to multiply the linear Zeeman term by the factor $g$ in Eq. (9b).

\subsection{Solutions near the Ionization Threshold}

\subsubsection{Classical and Semiclassical Approaches}

We consider first the treatment of the Zeeman effect by classical and semiclassical methods. An intrinsic limitation of any such approach is that it cannot offer an accurate account of the electron interaction with the ionic core. However, as we have stressed above, this interaction can often be dealt with in terms of a few quantum defect parameters. The major task set to any theoretical treatment of this problem is to give a good description of the electron motion in the region outside the ionic core; and in this region the quasiclassical criterion-that the variation of the potential over an electron de Broglie wavelength be sufficiently small-is largely satisfied for the magnetic field strengths and excitation energies of current experimental interest. Thus classical methods may be expected to give at least correct qualitative information on the observable quantities which do not depend strongly on the particular nature of the core. The most prominent of these, the nonintegral spacing of photoabsorption resonances near ionization thresholds was indeed first explained in semiclassical terms. ${ }^{(35,36)}$ The rather simple physical picture developed then has been elaborated upon, but has been essentially retained in all subsequent theoretical work.

The classical equation of motion for an electron in the field of an infinitely massive point nucleus of charge $+Z$ and a uniform magnetic field is (in c.g.s.-Gaussian units)

$$
m_{e} \ddot{\mathbf{r}}=-\frac{Z e^{2} \mathbf{r}}{r^{3}}-\frac{e}{c} \dot{\mathbf{r}} \times \mathbf{B}
$$

The correction for finite nuclear mass is insignificant for the kilogauss field 
strengths we treat in this section, as pointed out by $\mathrm{O}^{\prime}$ Connell. ${ }^{(31)} \mathrm{We}$ shall hereafter take the magnetic field to point along the Cartesian $z$ axis, $\mathbf{B}=B \hat{z}$. As pointed out by Gajewski, ${ }^{(37)}$ who seems to have performed the first systematic numerical study of the classical problem, Eq. (10) can be reduced to a parameter-free form by appropriate scaling of the space and time coordinates. With

$$
\begin{aligned}
\tau & =\omega t \\
\mathbf{R} & =\zeta \mathbf{r}
\end{aligned}
$$

where $\omega=e B / m_{e} c$ is the cyclotron frequency and $\zeta=\left(Z m_{e} c^{2} / B^{2}\right)^{-1 / 3}$, Eq. (10) becomes equivalent to

$$
\ddot{\mathbf{R}}=-\mathbf{R} / R^{3}-\dot{\mathbf{R}} \times \hat{z}
$$

The solutions to Eq. (12) are determined solely by the initial conditions $\mathbf{R}(\tau=0), \dot{\mathbf{R}}(\tau=0)$; if solutions for all such initial conditions are known, solutions of Eq. (10) appropriate to any values of $Z$ and $B$ are obtained from Eq. (11). Some appreciation of the magnitude of the scaling parameters can be had from the observation that the characteristic length $L=\zeta^{-1}$ is the radius of a sphere containing magnetic field energy $\left(B^{2} / 8 \pi\right) \cdot(4 \pi / 3) L^{3}$, which is comparable to the rest mass energy $m_{e} c^{2}$ of the electron. For the kilogauss fields discussed in this section, the cyclotron frequency $\omega$ is typically less than one hundred thousandth of the orbital frequency of the electron in the ground state of a hydrogen atom.

Two elementary constants of the motion are apparent in Eq. (12); an energy, $\varepsilon=\frac{1}{2} \dot{\mathbf{R}}^{2}-1 / R$; and an effective $z$ component of angular momentum, $\lambda_{z}=\hat{z} \cdot \mathbf{R} \times \dot{\mathbf{R}}-\frac{1}{2}(\hat{z} \times \mathbf{R})^{2}$. It is convenient to recast Eq. (12) in Hamiltonian form, utilizing $\varepsilon$ and $\lambda_{z}$. With $R$ and $\theta=\cos ^{-1}(\mathbf{R} \cdot \hat{z})$ denoting the usual polar coordinates of the vector $\mathbf{R}$, it is readily shown that Eq. (12) is equivalent to

$$
\frac{1}{2} \dot{R}^{2}+\frac{1}{2} R^{2} \dot{\theta}^{2}+\frac{\lambda_{z}^{2}}{2 R^{2} \sin ^{2} \theta}-\frac{1}{R}+\frac{\lambda_{z}}{2}+\frac{1}{8} R^{2} \sin ^{2} \theta=\varepsilon
$$

This is recognizable as the equation of motion of a particle in a combined spherical Coulomb potential $-1 / R$ and a cylindrical harmonic oscillator potential $\frac{1}{8}(R \sin \theta)^{2}$. The constant $\lambda_{z} / 2$ on the left-hand side of Eq. (13) corresponds to the linear Zeeman shift of quantum theory, which depends only on the $z$ component of angular momentum.

(a) Classical Theory of Planar Motion. No general solution to Eq. (13) has yet been discovered, nor are any additional constants of the motion 
known (though, as described below, there is some evidence for the existence of an approximate constant of the motion). However, one class of solutions of Eq. (13)-those with initial conditions $\theta=\pi / 2, \dot{\theta}=0$-can be carried out in closed form. Such solutions describe electron motions which are always confined to the plane $z=0$ which contains the nucleus and lies perpendicular to the direction of the field. It is clear that in this case the solution to Eq. (13) can be carried out in terms of elliptic integrals.

The relevance of this class of solutions to the spectroscopy of highly excited states in kilogauss fields is not immediately evident. In photoabsorption experiments the Rydberg electron emerges from the atom in a more or less spherical wave, and one would expect population of such planar motions to be quite improbable. Moreover, the planar orbits tend to be unstable with respect to small excursions out of the plane. It will be seen, however, that such motions, even though unstable, are of great importance in the photoabsorption spectrum. It is this feature which links the theory of the quadratic Zeeman effect with broader questions of dynamics which are raised below.

Without immediately addressing the question of orbital stability, we shall now examine the properties of the solutions of Eq. (13) for which $z=0$ always. Because of the smallness of the scaling parameter $\zeta-i$ it is about $\left(1350 a_{0}\right)^{-1}$ for hydrogen in a $50-\mathrm{kG}$ field-the quadratic term $\frac{1}{8} R^{2} \sin ^{2} \theta$ can be disregarded in determining the low-energy solutions, which are then essentially the orbits of a two-dimensional hydrogen atom. At high energies, on the other hand, the quadratic term becomes the most important part of the potential and the solutions must go over to those of the two-dimensional harmonic oscillator. The relevance of this equation to the interpretation of spectroscopic data, which covers an intermediate range of energies, can, however, only be determined by finding the allowed quantum-mechanical energy levels.

(b) Semiclassical Generalization: Interpretation of the Quasi-Landau Resonances. Edmonds ${ }^{(35)}$ first did this by applying the appropriate BohrSommerfeld quantization rule:

$$
\oint P_{\rho} d \rho=(n+1 / 2) h
$$

with $P_{\rho}$ being the classical momentum conjugate to the two-dimensional radial coordinate $\rho=\left(x^{2}+y^{2}\right)^{1 / 2}$. By numerical integration of Eq. (14) he found that, for hydrogen in a field of $24 \mathrm{kG}$, the energy levels near the ionization threshold are uniformly spaced, the separation between adjacent levels being approximately $1.58 \hbar \omega$. Moreover, Eq. (14) can be evaluated in an arbitrary plane $z=$ const (though, strictly speaking, the orbits are not solutions to the classical equations of motion unless $z=0$ ). This results 
in a set of energy levels which vary smoothly with $z$, going over to the harmonic oscillator levels as $z \rightarrow \infty$. It may also be remarked that the energy levels so calculated vary weakly with $z$ for small $z$. Thus it seems plausible to associate those prominent spectral features which are observed to be spaced by an energy of $\sim 1.5 \hbar \omega$ (Figure 1), with electron orbits which are largely confined to small values of $z$.

Of course, the actual position of an allowed energy level will depend strongly on the interaction of the Rydberg electron with the atomic core, which is not given realistically in this model. However, when cast in the form of a quantum defect, this residual interaction generally varies smoothly with energy near the ionization limit. This is because a small change in the kinetic energy of the Rydberg electron at large $r$ results in a much smaller proportional change in its kinetic energy near the residual core. Thus, as in the ordinary field-free quantum defect theory, the density of states in energy $\partial n / \partial E$ is determined principally by the form of the potential at large distances. Differentiation of Eq. (14) gives an expression for the density of states confined to the plane. Starace ${ }^{(36)}$ computed the resulting integral numerically and obtained the results shown in Figure 2 . The basic result of interest can be seen from the expression

$$
\frac{\partial n}{\partial E}=\frac{2}{\pi \hbar \omega} \int_{-1}^{1} \frac{d z(z+\gamma)}{\left[\left(1-z^{2}\right)\left(z^{2}+4 \gamma z+6 \gamma^{2}+1-4 \varepsilon\right) /\left(\rho_{2}-\rho_{1}\right)^{2}\right]^{1 / 2}}
$$

where $\varepsilon=8 E /\left(m_{e} \omega^{2}\right), \rho_{2}$ and $\rho_{1}$ are the greater and lesser of the two classical turning points, and $\gamma=\left(\rho_{2}+\rho_{1}\right) /\left(\rho_{2}-\rho_{1}\right)$. The inner turning point $\rho_{1}$ is (for the kilogauss fields of interest) determined solely by the Coulomb potential and the centrifugal barrier, and so is of the order of unity. The outer turning point $\rho_{2}$ is, on the other hand, determined by the relative magnitude of the Coulomb and quadratic potentials, and for energies near threshold, $\rho_{2} \simeq 2 / \zeta$. Thus, $\gamma$ is very nearly equal to 1 , with only weak dependence on the $z$ component of angular momentum $m \hbar$ and the magnetic field strength; for $m=0$ we have $\gamma=1$ independent of the field. If we take $\gamma=1$ and consider the case $E=0$, the integral in Eq. (15) can be evaluated by elementary means and yields

$$
\frac{\partial n}{\partial E}=\frac{2}{3 \hbar \omega}
$$

When $E \gg \hbar \omega$, Eq. (15) reduces to the result for the two-dimensional oscillator

$$
\frac{\partial n}{\partial E}=\frac{1}{\hbar \omega}
$$



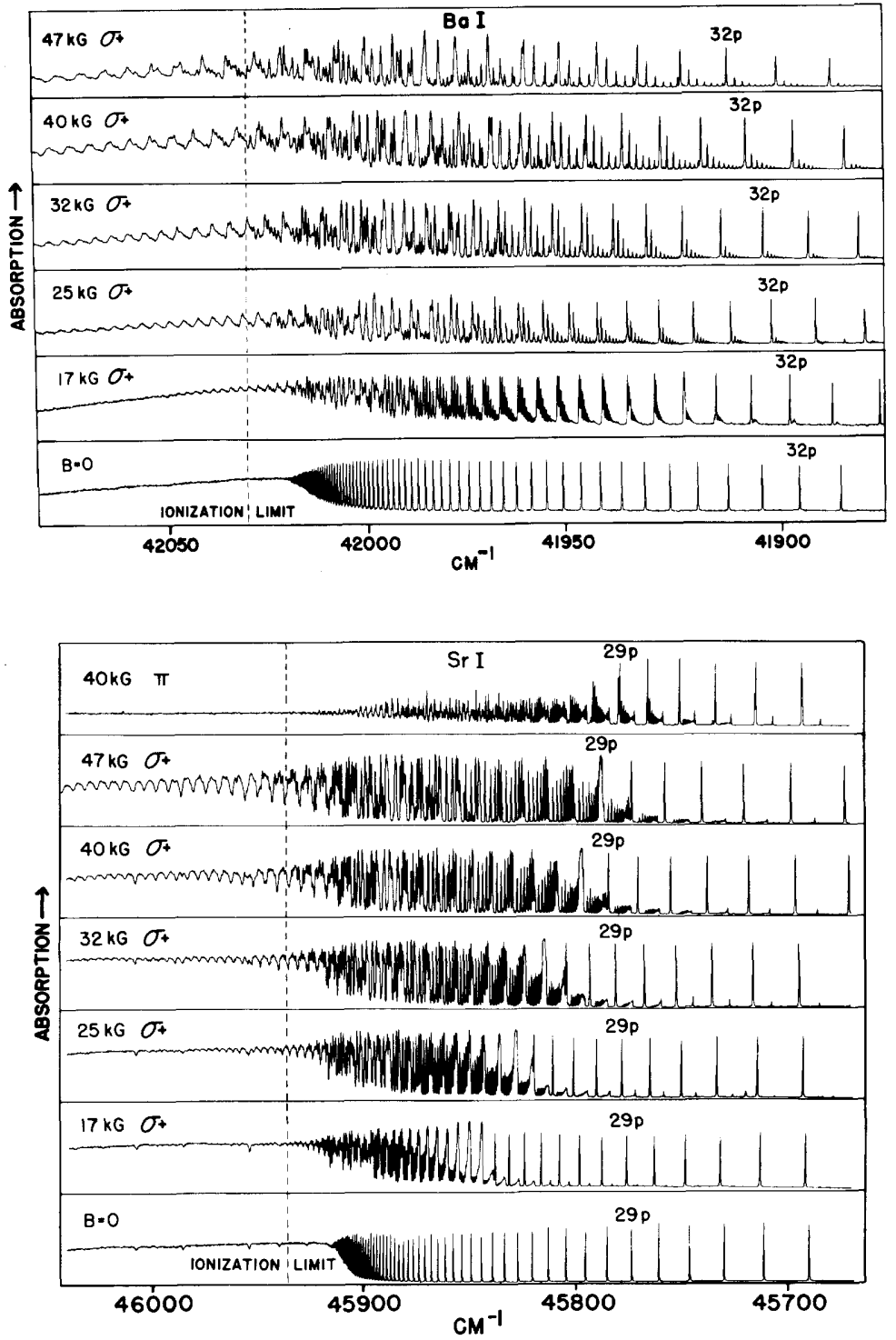

Figure 1. (a) Microdensitometer recording of the plate transmission of the $\mathrm{Ba}$ principal series with magnetic field strengths $B=47,40,32,25$, and $17 \mathrm{kG}$ ( $\sigma^{+}$polarization) and $B=0$ (from Ref. 11). (b) Microdensitometer recording of the plate transmission of the $\mathrm{Sr}$ principal series. The top spectrum is that for $\pi$-polarization with $B=40 \mathrm{kG}$. The next five spectra are for $\sigma^{+}$ polarization with $B=47,40,32,25$, and $17 \mathrm{kG}$. The last spectrum is for $B=0$. (From Ref. 11.) 


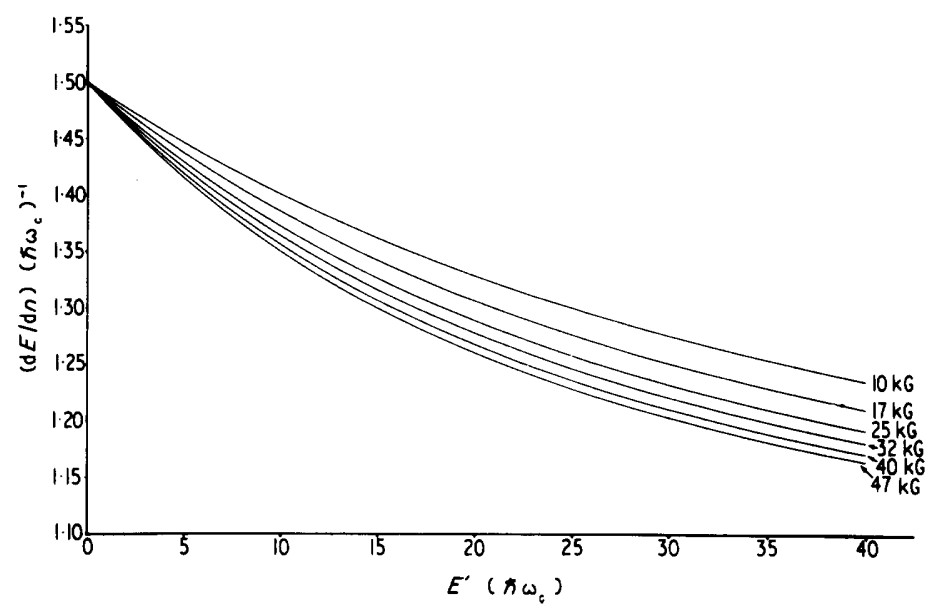

Figure 2. WKB results for the quasi-Landau level separations $\partial E / \partial n$ [cf. Eq. (15)] plotted vs. energy above threshold for various magnetic field strengths. Both axes are in units of the cyclotron energy $\hbar \omega$. Note that $\partial E / \partial n=1.5$ at threshold regardless of field strength. (From Ref. 36.)

The density of states increases monotonically as the energy increases from the ionization threshold. The experimental spectra show rather broad features, with some secondary structure, above the ionization limit (cf. Figure 1). However, it is possible to determine the positions of the centers of the major peaks without too much ambiguity. Garton et $a l .{ }^{(38)}$ have done this and found that their measurements of the quasi-Landau energy spacings are in quite good agreement with Starace's ${ }^{(36)}$ results for $(\partial E / \partial n)$ using the WKB approximation of Eq. (15), as shown in Figure 3. Setting $\gamma=1$ again, the total number $N_{T}$ of bound energy levels in the plane with given angular momentum $m \hbar$ can be determined as

$$
N_{T}=\frac{1}{2}+\frac{2^{-1 / 3} \Gamma(1 / 3)}{[\Gamma(2 / 3)]^{2}} \frac{m_{e} \omega}{\hbar \zeta^{2}}
$$

For example, $N_{T} \approx 43$ for hydrogen in a $50-\mathrm{kG}$ field. The only dependence on $m$ comes through the ratio $\gamma$. This number $N_{T}$, if correctly interpreted, is also in rough agreement with "experimental" data, as will be seen below.

(c) Three-Dimensional Orbits. The quasi-classical approach thus accounts for some systematic features of the experimental spectra. It cannot, however, provide any definite prediction of line widths or the distribution of oscillator strength, and it is clear from the data that there are many more lines of spectroscopic importance than can be accounted for in this 
a)

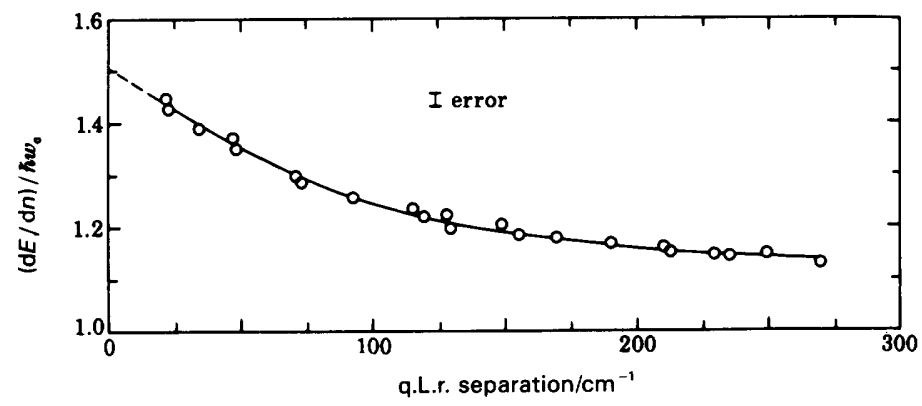

b)

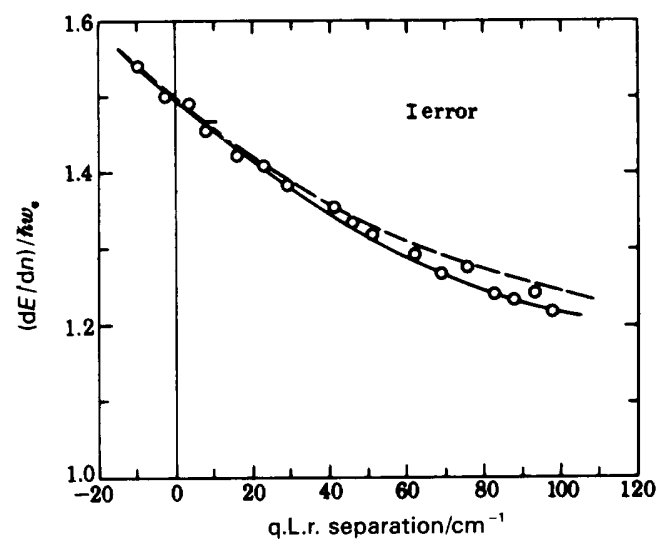

Figure 3. Comparison of the measured quasi-Landau energy level separations of Garton et al. (Ref. 38), shown by open circles, with predictions of the WKB result for $(\partial E / \partial n) / \hbar \omega_{c}$ of Starace (Ref. 36). (a) Barium in a $46.9-\mathrm{kG}$ field; WKB result [cf. Eq. (15)] indicated by the solid curve. (b) Strontium in a 48.3-kG field; WKB result [cf. Eq. (15)] indicated by the dashed curve. The solid curve in (b) shows the trend of the experimental data. The difference between the WKB result and experiment is about twice the estimated experimental error in the case of Sr. The abscissas indicate the energy above the threshold in $\mathrm{cm}^{-1}$.

way. An alternative, purely classical, approach to the problem has been taken by Edmonds and Pullen, ${ }^{(39)}$ who do not restrict the electron's motion to the plane. They proceed from an equation similar to Eq. (13), with, however, a different choice of scaling parameters: the unit of distance being $2 / \zeta$ and the unit of time chosen as $4 / \omega$. Their unit of energy is twice the electrostatic potential between a proton and electron separated by a distance $\rho_{0}$. In these units the magnitude of Planck's constant depends on the strength of the field, but typically $\hbar \approx 10^{-2}$ for fields in the range 10-50 kG. Since classical and quantum mechanics coincide when $\hbar \rightarrow 0$, this magnitude may be taken as a rough measure of the validity of a classical treatment. 


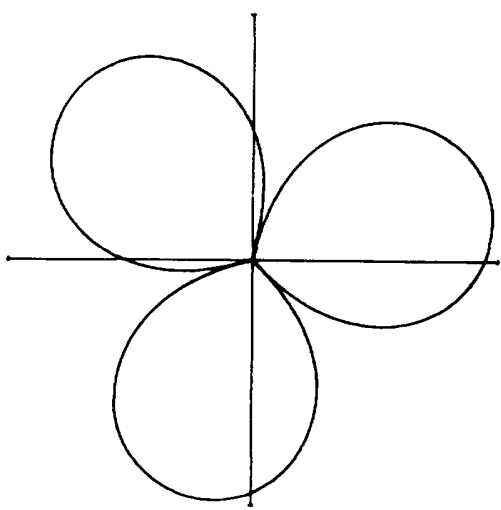

Figure 4. The classical planar orbit for an electron with zero energy in combined Coulomb and magnetic fields. (From Ref. 39.)

Edmonds and Pullen have done considerable numerical investigation of the classical solutions. One novel feature of their approach is the use of a regularization transformation of the space and time coordinates, of a type familiar in celestial mechanics, which removes the singularity in the Coulomb potential near the nucleus and thus greatly improves the numerical stability of the calculation. Their interpretation of the results is founded on the application of the correspondence principle. If the classical motion is periodic with angular frequency $\omega_{\mathrm{cl}}$, and if $\omega_{\mathrm{cl}}$ does not vary appreciably over a given energy interval, then the spacing of quantum mechanical energy levels within that interval must be $\Delta E=\hbar \omega_{\mathrm{cl}}$. A trefoil classical planar orbit of zero energy and small angular momentum is shown in Figure 4. The angular frequency of this motion is $\omega_{\mathrm{cl}}=\frac{3}{2} \omega$. Edmonds and Pullen report that the angular frequency of classical orbits of this type does not vary by more than $5 \%$ throughout the energy range (in their units) $-0.1<$ $E<0.01$. Thus the correspondence principle can be invoked with reasonable confidence, and yields the same result as the WKB treatment for the threshold spacing.

However, for any given energy there are many orbits which are not periodic so that this analysis does not apply. By computing surfaces of section $^{(40)}$ for the classical trajectories, Edmonds and Pullen have obtained a comprehensive catalogue of the types of orbit which exist in different ranges of energy. For energies $E<-1 / 2$ in their units, they find that nearly all motions are regular (in the sense of Percival ${ }^{(41)}$ ), i.e., they can be regarded as the resultant of superposed periodic motions. As the energy rises above this value, an increasing volume of phase space is filled by irregular orbits and at the ionization limit $E=0$ the entire volume of phase space seems to be occupied by irregular trajectories. Though none of these are strictly 
periodic, some consist of motions in which the electron returns to the region near the proton a number of times before drifting away. These may be imagined to have important quantum mechanical analogs, i.e., states with small but nonnegligible oscillator strength which might be associated with the fine structure in the photoabsorption cross sections seen above the ionization threshold. A very qualitative but plausible method of assessing the quantum mechanical significance of such orbits has been put forward. In photoabsorption the electron will be ejected at some angle relative to the plane perpendicular to the field. If it is placed in a quasi-periodic orbit, it will return at some later time to the neighborhood of the nucleus. The inverse of the distance of closest approach upon return to the nucleus may be supposed to be a measure of both the stability of the quasiperiodic motion and of its associated oscillator strength. Figure 5 shows the spectrum of this inverse impact parameter as a function of the ejection angle, for a class of quasiperiodic orbits calculated by Edmonds and Pullen. ${ }^{(39)}$ Firm quantitative results have not however been produced in this framework.

Edmonds and Pullen ${ }^{(39)}$ have also provided a classical interpretation of the effect of the motional electric field on the spectrum near threshold. This field has not been of obvious importance in experiments on heavy atoms. In photoabsorption by $\mathrm{Li}$ vapor at a temperature of $\approx 800^{\circ} \mathrm{K}$,

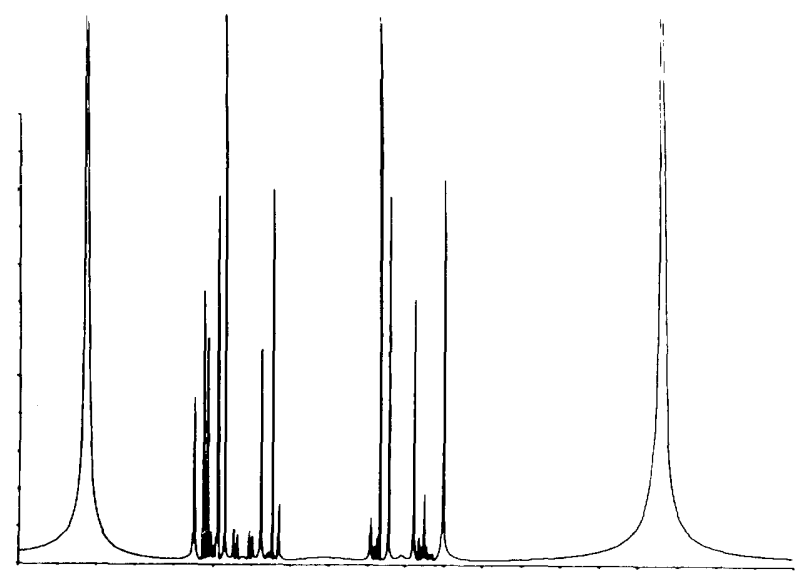

Figure 5. A representation of periodic classical orbits at zero energy. The abscissa is one of the initial regularized momenta of the system, corresponding to the initial angle of the orbit with respect to the plane $z=0$. The ordinate is the inverse of the impact parameter on the return journey. (From Ref. 39.) 
however, it has been observed ${ }^{(13)}$ that the spacing between major features is $\frac{1}{2} \hbar \omega$ rather than the semiclassical value $\frac{3}{2} \hbar \omega$. This difference was attributed by Crosswhite et $_{\text {al. }} .^{(15)}$ to the presence of the motional electric field, using arguments summarized in Section 5 of this paper. Edmonds and Pullen ${ }^{(39)}$ have shown that a motional Stark field of the appropriate magnitude splits the trefoil orbit of Figure 4 into another three-lobed periodic orbit, only one lobe of which passes through the nucleus (Figure 6). As the frequency of return is thus $1 / 3$ the value without the electric field, the correspondence principle gives a $\frac{1}{2} \hbar \omega$ spacing.

NOTE (added in proof): Since this article was written, another investigation of the quadratic Zeeman effect by classical methods was reported by Robnik. ${ }^{(138)}$ Though it was applied to fields of astrophysical strength, this work employed methods similar to those of Edmonds and Pullen and yielded results consistent with theirs. In particular, Robnik also observed a transition to occur between regular and irregular motion at a critical energy.

(d). Classical and Semiclassical Perturbation Theory: Coupled Motion of Runge-Lenz and Angular Momentum Vectors. An elegant treatment of the classical problem by perturbation theory has been recently carried out by Goebel and Kirkman. ${ }^{(42)}$ If the magnetic term of Eq. (12) is suppressed,

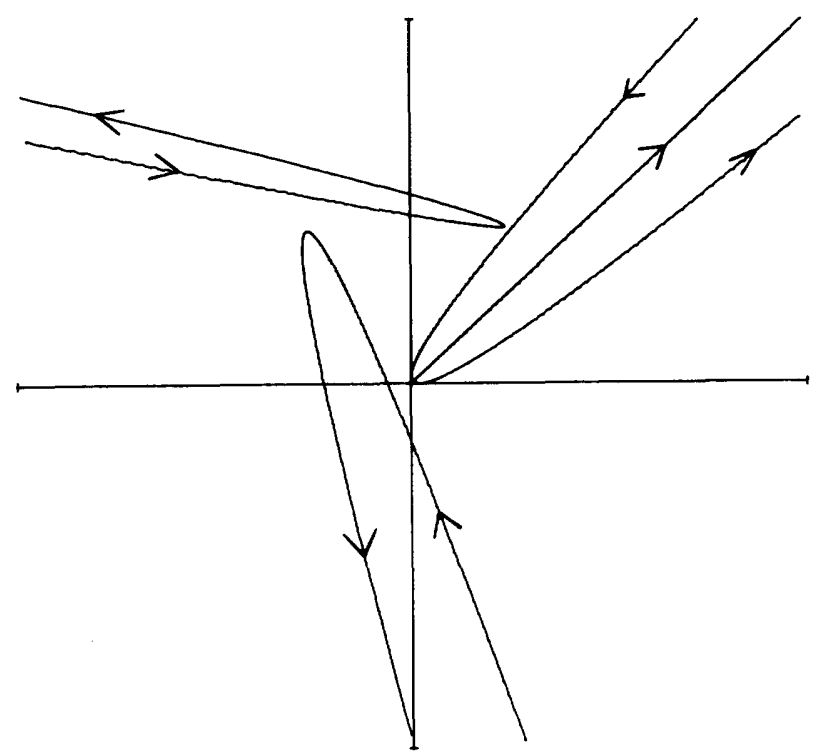

Figure 6. The disruption of the trefoil orbit of Figure 4 by a motional electric field. (From Ref. 39.) 
we recover the ordinary Kepler equation. This exhibits seven constants of the motion: the energy $\varepsilon$, the angular momentum vector $\boldsymbol{\lambda}=\mathbf{R} \times \dot{\mathbf{R}}$ and the Runge-Lenz vector $\boldsymbol{\alpha}$

$$
\boldsymbol{\alpha}=(-2 \varepsilon)^{-1 / 2}[(\dot{\mathbf{R}}+\lambda-\mathbf{R}) / R]
$$

where $\varepsilon<0$ for bound orbits. These are not all independent since

$$
\lambda \cdot \boldsymbol{\alpha}=0, \quad \lambda^{2}+\alpha^{2}=(-2 \varepsilon)^{-1}=N^{2}
$$

Goebel and Kirkman calculate the average value of the diamagnetic potential $\frac{1}{8} R^{2} \sin ^{2} \theta$ over an unperturbed Kepler orbit and find that

$$
U=\left\langle\frac{1}{8} R^{2} \sin ^{2} \theta\right\rangle=\frac{N^{2}}{16}\left[5\left(\alpha^{2}-\alpha_{z}^{2}\right)+\lambda^{2}+\lambda_{z}^{2}\right]
$$

If $U$ is then regarded as an effective potential, equations of motion for the vectors $\lambda$ and $\alpha$ may be obtained. If we substitute $U$ for $\frac{1}{8} R^{2} \sin ^{2} \theta$ in Eq. (13), we obtain an effective classical Hamiltonian function

$$
H=H_{c}+\frac{\lambda_{z}}{2}+U
$$

where $H_{c}$ is the ordinary Coulomb Hamiltonian. Recall that the classical equation of motion for a function $f(q, p)$ of canonical coordinates $q$ and their conjugate momenta $p$ is

$$
f=\{f, H\}=\sum_{i} \frac{\partial f}{\partial q_{i}} \frac{\partial H}{\partial p_{i}}-\frac{\partial f}{\partial p_{i}} \frac{\partial H}{\partial q_{i}}
$$

the brackets being the familiar Poisson brackets. As is well known, they are the classical analog of the quantum mechanical commutation brackets. ${ }^{(43)}$ The equations of motion which result for $\boldsymbol{\lambda}$ and $\boldsymbol{\alpha}$ by applying Eqs. (22) and (23) are described as follows. Since $\boldsymbol{\lambda}, \boldsymbol{\alpha}$ are constants of motion for the Coulomb problem, $\left\{\boldsymbol{\lambda}, H_{c}\right\}=\left\{\boldsymbol{\alpha}, H_{c}\right\}=0$. The term $\lambda_{z} / 2$ results in a uniform precession of $\boldsymbol{\lambda}$ and $\boldsymbol{\alpha}$ about the direction of the field, with frequency $1 / 2$ with respect to the scaled time $\tau$ (recall the quantum mechanical description of angular momentum as the generator of rotations). Since $U$ is quadratic in the components of $\boldsymbol{\lambda}$ and $\boldsymbol{\alpha}$, its contribution to their time derivatives is an asymmetric bilinear form; in fact, the equations 
of motion resulting from $U$ are isomorphic to the Euler equations for a rotating four-dimensional top. We shall not reproduce the equations in detail here, but will only give a brief summary of the properties of their solutions. They exhibit four constants of motion: $\lambda_{z}, U$, and the two conditions of Eq. (20). Thus working with this system of equations is the classical analog of degenerate perturbation theory in quantum mechanics (discussed below), since from Eq. (20) $N$ may be readily identified with the principal quantum number $n$.

The qualitative behavior of the orbits computed in classical perturbation theory is as follows. Recall that, in field free hydrogen, $\boldsymbol{\alpha}$ is directed along the major axis of the elliptical orbit and its magnitude is proportional to the eccentricity of the orbit. For low-energy perturbed orbits, the major axis oscillates in a plane parallel to the field, and stays near the $z$ axis, i.e., $\alpha_{z}$ remains either always positive or always negative. For higher energies the orbital axis oscillates about the $z=0$ plane; the highest energy orbit of fixed $N$ is confined to that plane. In addition, there is uniform precession of the orbital axis about the direction of the field.

Goebel and Kirkman ${ }^{(42)}$ have carried out semiclassical quantization of the perturbed system by requiring the resulting action integrals to be multiples of Planck's constant. The resulting energy spectrum resembles that of a double-well potential problem. Low-lying states are doubly degenerate, being confined to either of the wells. The highest state lies on the barrier separating the wells.

NoTE (added in proof): After the completion of this article, another treatment by classical secular perturbation theory was reported by Soloviev. ${ }^{(139)}$ He obtains equations of motion equivalent to those of Geobel and Kirkman for the Runge-Lenz and angular momentum vectors. Their associated quantum mechanical spectrum is surveyed by applying BohrSommerfeld quantization to the component of angular momentum perpendicular to the magnetic field [i.e., our Eq. (14) with $\rho$ replaced by $\theta$ ]. The form of the quantization integral suggests a double-well potential for the low-lying states; however, for the highest states in the spectrum, the effective potential takes the form of a single well which confines the electron orbit to the angular range $\theta=\pi / 2 \pm \sin ^{-1}(1 / \sqrt{5})$, i.e., about the plane $z=0$. This is consistent with the non-perturbative treatment described in the next subsection, and with the numerical evidence discussed in Section 2.2.2d.

(e) Wave Propagation along a Potential Ridge. In the results of Goebel and Kirkman ${ }^{(42)}$ we see the localization of some orbits in the plane $z=0$. Fano ${ }^{(44,45)}$ has recognized this aspect as being common to a large class of problems in which electron motion takes place in the presence of a rising "ridge" of a potential surface. The general semiclassical theory he has developed for such systems remains yet to be applied in detail to the 
quadratic Zeeman problem. Nevertheless, even in its present stage of development this theory provides some account of the mechanism responsible for the novel results appearing in large brute-force quantum mechanical calculations. Since many of its implications have not been worked out, a full review here of this theory would be premature. A brief discussion of its elements may, however, help fill in the background for quantum mechanical considerations.

In the atomic units which will be employed in the remainder of this section, the quadratic Zeeman Hamiltonian is

$$
H=\frac{1}{2} \mathbf{p}^{2}-Z / r+\frac{1}{2} \beta^{2} r^{2} \sin ^{2} \theta
$$

with $\beta=\omega / 2$ a.u. and $\theta$ the polar angle of the electron position vector as defined previously. The linear Zeeman shift is here understood to be included in the energy eigenvalue. At fixed electron-nuclear distance $r$, the potential in Eq. (24) is at a maximum in the plane $\theta=\pi / 2$. The full potential surface (Figure 7) takes the form of a ridge straddling this plane. The azimuthal motion of the electron is trivial, so we need consider only the two degrees of freedom associated with the coordinates $r$ and $\xi=$ $\theta-\pi / 2$. This choice of the angular parameter $\xi$ is motivated by the implication from spectroscopic data that motions involving only small displacements from the ridge at $\xi=0$ are of greatest importance. Then by expanding the classical Hamilton-Jacobi equation appropriate to the system (24), and applying a linearization transform analogous to that employed by Wannier, ${ }^{(46)}$ Fano identifies two bundles of trajectories $\xi_{ \pm}(r)$. Trajectories of diverging type $\xi_{+}(r)$ move gradually off the ridge as $r$ increases; the converging trajectories $\xi_{-}$remain on the ridge. Individual trajectories within a bundle are described by a scale parameter $T$ which is determined

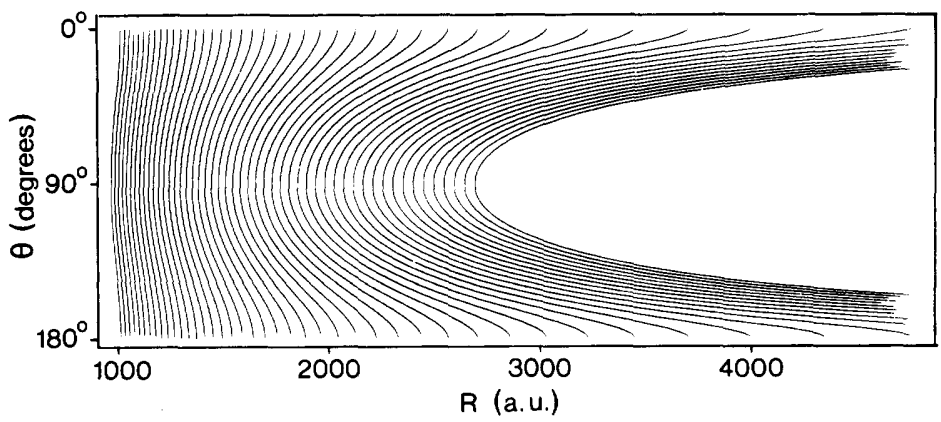

Figure 7. Equipotential surfaces for hydrogen in a $47-\mathrm{kG}$ field. Abscissa: electron-proton distance in atomic units; ordinate: angle between electron position vector and magnetic field. The equipotential lines are spaced by one fifth of the cyclotron energy, the rightmost corresponding to zero energy. (From Ref. 60.) 
by initial conditions; specifically, the diverging and converging trajectories with a particular value of $T$ are given, to lowest order, by

$$
\xi_{T \pm}=\operatorname{Tr}^{\xi_{ \pm}(r)}
$$

When the two exponents $\zeta_{ \pm}(r)$ do not vary rapidly with $r$, they are the roots of a quadratic equation; in general they are the solutions to a simple Riccati differential equation. The radial dependence of these exponents is indicated in Figure 8.

Since $\zeta_{+} \rightarrow 0$ at small $r$, a bound electron excited by a photon will necessarily move to large $r$ on a diverging trajectory. As the classical turning point is approached, the diverging exponent $\zeta_{+}$begins to increase rapidly, and some fractional transfer of the electron wave function to a converging trajectory will occur. At the turning point the diverging trajectory veers sharply away from the ridge; the converging trajectory, on the other hand, undergoes elastic reflection. Thus that fraction of the electronic wave function on the converging trajectory travels back towards the nucleus; that on the diverging trajectory is scattered to large angles $\xi$, leading to ionization if there is sufficient energy. The standing waves which are associated with the structures seen in photoabsorption may then be visualized as being built up by repeated passes from small to large $r$ along the appropriate trajectories. The actual magnitude of the coupling between converging and diverging trajectories has not yet been established, so it is

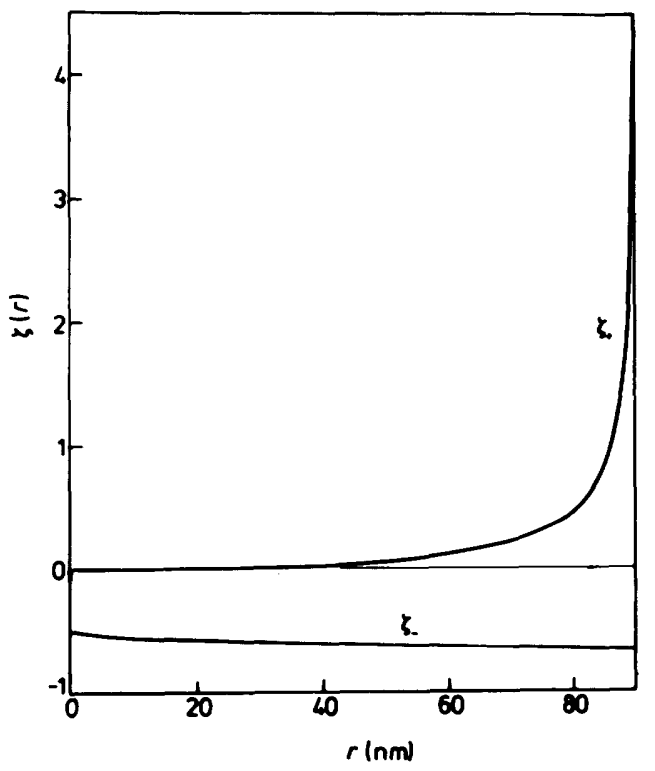

Figure 8. Characteristic exponents of diverging and converging trajectories for hydrogen in a $94-\mathrm{kG}$ field, as a function of electronproton distance in units of $10^{-9} \mathrm{~m}$. (From Ref. 45 .) 
not presently possible to make any definite statement about the energies or widths of the bound levels and continuum resonances. However, this formulation provides a plausible mechanism for the generation of standing wave motion along the ridge. Some qualitative conclusions about its further development can be inferred from the "experimental" data provided by large quantum mechanical calculations.

\subsubsection{The Quantum Theoretical Treatment}

(a) General Aspects. We continue on the trail of the Zeeman effect for moderate field strengths $(10-100 \mathrm{kG})$, though the bulk of quantum theoretical work has been done for much stronger fields $\left(10^{4}-10^{9} \mathrm{kG}\right)$. These two cases are sufficiently different to warrant a separate section on the strong fields. For although in both cases one must deal with a nonseparable Hamiltonian not usually amenable to a perturbative treatment, in the strong field case the density of levels in the energy region of interest is very much smaller than that arising in the case of a moderate field. Thus, any calculation of a standard type applied to the case of moderate field strength, is necessarily large, and is bound to produce many solutions of little relevance to conventional spectroscopy.

We return to Eq. (24), which gives the Hamiltonian for electron motion in the region $r>r_{0}$, where $r_{0}$ is the size of the residual core. For a field of $47 \mathrm{kG}, \beta=10^{-5}$, which we shall take to be a typical value in the remainder of this section. It is clear that for a significant distance beyond the core, $r_{0}<r<r_{1}$, the magnetic potential is negligible in comparison to the Coulomb and centrifugal potentials; $r_{1}$ being, say, several hundred Bohr radii. Then in the region $r_{0}<r<r_{1}$ the wave function may be expanded in the form

$$
\psi(\mathbf{r})=\sum_{\alpha} A_{\alpha} \sum_{i} \Phi_{i} U_{\alpha i}\left[f_{i}(r) \cos \pi \mu_{\alpha}-g_{i}(r) \sin \pi \mu_{\alpha}\right]
$$

Here the terms in the summation over $i$ are those determined by the MQDT treatment of the field-free atom (though they may be slightly modified by the Zeeman effect on the core) as defined subsequently in Section 4 ; in the case of hydrogen, $\Phi_{i}$ reduces to a spherical harmonic of the electron position vector, $f_{i}$ is the appropriate Coulomb wave function, and $\mu_{\alpha} \equiv \mu_{l}=0$. The $A_{\alpha}$, on the other hand, are determined by boundary conditions imposed at large $r$; that is, they determine the superposition of eigenchannels at small $r$ which leads to a wave function vanishing at large $r$ (or one which describes electron escape along the $z$ axis, if the energy is sufficient). They will be dependent on the energy and the eigenchannel parameters, in a manner which has not yet been generally determined. 
(b) Degenerate Perturbation Theory in Spherical Coordinates. We shall now discuss the computation of the $\boldsymbol{A}_{\alpha}$ in the context of perturbation theory, which is applicable when the states considered do not extend far beyond $r=r_{1}$. The calculations involved are relatively trivial but, at least for hydrogen, bring out a number of features which do appear to persist beyond the strict range of validity of perturbation theory.

Hydrogen in the absence of a field has bound states grouped in degenerate manifolds with given principal quantum number $n$. The $n$th manifold is $n^{2}$-fold degenerate (neglecting the electron spin, which is for our purposes a constant of the motion ${ }^{(47)}$, containing all states with orbital momenta $l<n$. Taking $V=\frac{1}{2} \beta^{2} r^{2} \sin ^{2} \theta$ as a perturbation, it is found that the matrix elements of $V$ within an $n$ manifold are of order $\beta^{2} n^{4}$. The energy difference between adjacent manifolds is of order $n^{-3}$. Thus if $\beta^{2} n^{7}$ is small, perturbation theory can be carried out within a single $n$ manifold; on the other hand, it is apparent that as $n$ becomes large a significant number of manifolds may be coupled by the magnetic interaction. The density of states and the strength of the perturbation increase together with $n$. We shall for the moment consider only the cases in which perturbation theory can be carried out within a degenerate manifold.

As $m=1 \cdot \hat{z}$ is a constant of the motion, one treats separately each submanifold of states with fixed $m$. Each of these is further split by the conservation of parity, so that for each manifold of fixed $n, m$ one has a separate calculation to perform for states of even and odd parity: roughly equal numbers of states of each parity $[\approx(n-|m|) / 2]$ are involved. For fixed $n, m$, and parity the perturbation $\frac{1}{2} \beta^{2} r^{2} \sin ^{2} \theta$ is diagonalized. The resulting eigenstates are independent of the field strength, and the perturbed energies scale with field strength simply as $\beta^{2}$.

As $\sin ^{2} \theta$ is a combination of tensors of rank 0 and 2 with respect to rotations, the matrix elements $\left\langle n l^{\prime} m|V| n l m\right\rangle$ necessarily vanish when $\left|l-l^{\prime}\right|>2$. This enables the perturbation matrix to be written in tridiagonal form. The angular matrix elements $\left\langle l^{\prime} m\left|\sin ^{2} \theta\right| l m\right\rangle$ are determined straightforwardly by angular momentum algebra; the radial matrix elements, which are independent of $m$, are given by ${ }^{(17)}$

$$
\begin{aligned}
\left\langle n l^{\prime}\left|r^{2}\right| n l\right\rangle & =\frac{n^{2}}{2 Z^{2}}\left[5 n^{2}+1-3 l(l+1)\right] \quad l^{\prime}=l \\
& =\frac{5 n^{2}}{2 Z^{2}}\left\{\left(n^{2}-l_{>}^{2}\right)\left[n^{2}-\left(l_{>}-1\right)^{2}\right]\right\}^{1 / 2} \quad l^{\prime}=l \pm 2
\end{aligned}
$$

The energies and eigenfunctions of hydrogenic degenerate perturbation theory can be determined routinely for $n$ values of up to several thousand. (Though, for hydrogen in a $50-\mathrm{kG}$ field, degenerate perturbation theory 
ceases to be valid at around $n=20$ ). Methods alternative to straightforward matrix diagonalization have been put forward by Killingbeck ${ }^{(48)}$ and Avron et al. ${ }^{(49)}$ These, however, appear to be more appropriate to the consideration of low-lying states in very strong fields, and so are dealt with in Section 2.2.3.

We give here only a brief summary of the results obtained in numerical solution of the perturbation equations. It is found that the highest and lowest eigenvalues are nearly equally spaced in energy, in agreement with the semiclassical results of Goebel and Kirkman. ${ }^{(42)}$ If the oscillator strengths for dipole transitions from, e.g., the hydrogen $1 s$ ground state to perturbed Rydberg states are computed, a significant difference between the spectra of states with different values of $m$ is observed. If the parity of the perturbed manifold is equal to $(-1)^{|m|}$, the oscillator strength tends to be concentrated in a single state (see the lowest line cluster of Figure $10)$. For parity equal to $(-1)^{|m|+1}$, on the other hand, the oscillator strength tends to be spread more or less uniformly among all the perturbed states (Figure 9). Since the total oscillator strength associated with a given manifold must be equal to the field-free value of the oscillator strength-this is the classical concept ${ }^{(4)}$ of "spectroscopic stability"- the spectrum of states in the latter category will be more diffuse. It should be recalled that these states necessarily have a node in their wavefunctions in the plane $z=0$. Thus they are less confined by the magnetic field than the states with parity $(-1)^{|m|}$, which necessarily display an antinode along $z=0$ (the lowest clusters of Figure 10 are an example). It will be seen below that in fact the wave function of the strongest line of that manifold of states attains its maximum in the plane.

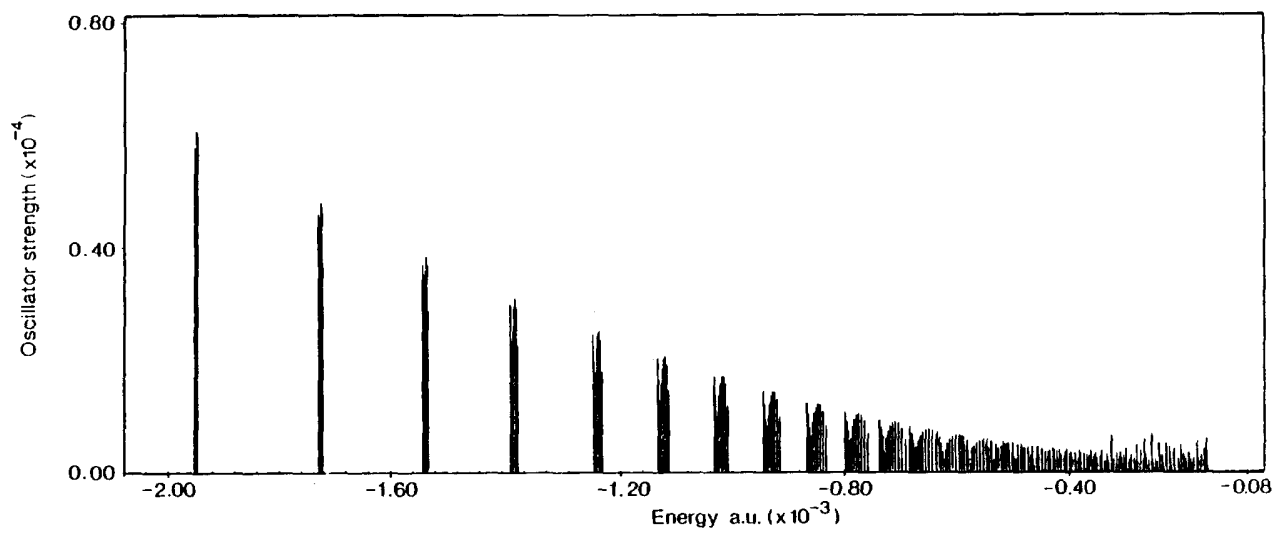

Figure 9. Partial photoabsorption spectrum of ground state hydrogen in a magnetic field of $47 \mathrm{kG}$. The light is polarized linearly along the field axis ( $\pi$ polarization). The abscissa is the absolute energy of the final states involved; the ordinate, the oscillator strength. The lowest cluster of lines describes the perturbed $n=23$ manifold. (From Ref. 59.) 


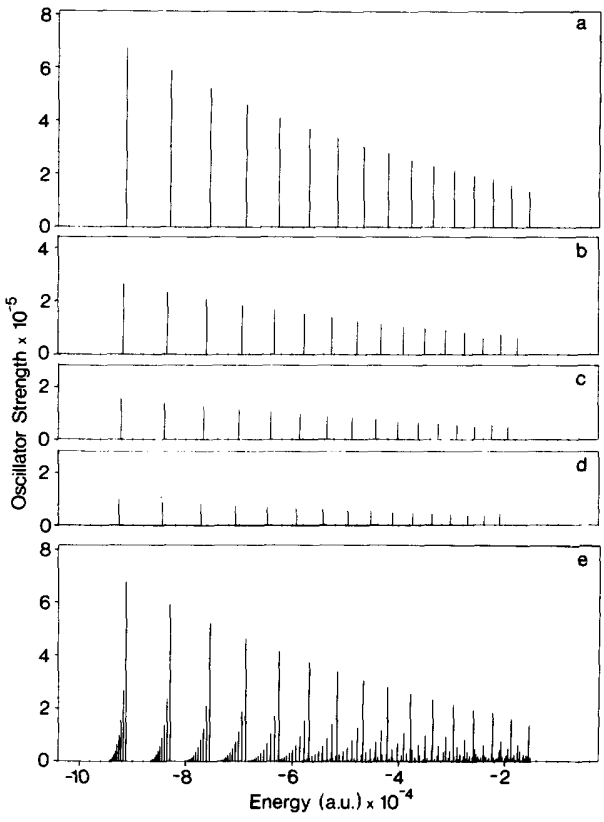

Figure 10. The $\sigma$ polarization absorption spectrum of hydrogen in the same conditions as of Figure 9. Frame (e) shows the net oscillator strength distribution in this energy region; frames (a)-(d) depict the principal, second, third, and fourth series, respectively. (From Ref. 60.)

For nonhydrogenic atoms similar calculations may be carried out, though the zero-field degeneracy of levels is broken by the presence of quantum defects. One is then faced with evaluating the radial matrix elements of Eq. (27) with respect to principal quantum numbers $n^{*}=n-\delta$ which are nonintegral. This can be done to very good accuracy by using only the asymptotic form of the Coulomb wavefunction [from Eq. (26)], a method analogous to that employed by Bates and Damgaard ${ }^{(50)}$ for the computation of oscillator strengths. The diagonal matrix elements of Eq. (27) turn out to be nearly equal to those obtained simply by substituting $n^{*}$ for $n$; the off-diagonal elements are not so easy to summarize succinctly (see, however, Picart et al. ${ }^{(51)}$ ), but their calculation presents no real difficulty. This method has been applied by Crosswhite et al $^{(15)}$ to portions of the lithium spectrum, by Edmonds and Kelley to barium, and by Clark and Taylor to magnesium (both unpublished). The magnesium spectrum is of some particular interest in that in the range $n \cong 5-12$ the quantum defects for $p$ and $f$ states differ by nearly unity, ${ }^{(52,53)}$ so the zero-field degeneracy is almost recovered.

(c) Alternative Perturbation Treatment in Parabolic Coordinates: The Role of the Runge-Lenz Vector. An alternative formulation of degenerate perturbaton theory for hydrogen reveals several striking new features, and indeed nearly allows the perturbation equations to be solved in closed form as shown in recent work by Goebel and Kirkman. ${ }^{(42)}$ As is well known, 
the field-free equations of motion for hydrogen are separable in the parabolic coordinates $\xi=r+z$ and $\eta=r-z$. Their eigensolutions take the form

$$
\begin{aligned}
\left|n_{1} n_{2} m\right\rangle & =f_{n_{1}}(\xi) f_{n_{2}}(\eta) \frac{e^{i m \phi}}{(2 \pi)^{1 / 2}} \\
n & =n_{1}+n_{2}+|m|+1
\end{aligned}
$$

where $n_{1}, n_{2}$ are, respectively, the number of nodes in the $\xi$ and $\eta$ components of the wave function, and assume all positive values consistent with Eq. (28); the $f$ 's are products of Laguerre polynomials and exponentials. ${ }^{(54,55)}$ Since the parity operator $P$ interchanges $\xi$ and $\eta$, these wave functions are generally not eigenfunctions of parity; rather

$$
P\left|n_{1} n_{2} m\right\rangle=(-1)^{m}\left|n_{2} n_{1} m\right\rangle
$$

In the parabolic coordinates the diamagnetic perturbation takes the form

$$
V=\frac{1}{2} \beta^{2} r^{2} \sin ^{2} \theta=\frac{1}{2} \beta^{2} \xi \eta
$$

so that, in contrast to the spherical system, $V$ is both linear and symmetric in the separate coordinates. Consequently, within the degenerate manifold $n_{1}+n_{2}=n-|m|-1=$ constant

$$
\begin{gathered}
\left\langle n_{1} n_{2} m|V| n_{1}^{\prime} n_{2}^{\prime} m\right\rangle=\left\langle n_{2} n_{1} m|V| n_{2}^{\prime} n_{1}^{\prime} m\right\rangle \\
\left\langle n_{1} n_{2} m|V| n_{1}+i n_{2}-i m\right\rangle=0 \quad \text { for }|i|>1
\end{gathered}
$$

The specific forms of the matrix elements $\left\langle n_{1} n_{2} m|\xi \eta| n_{1}+i n_{2}-i m\right\rangle \operatorname{are}^{(54)}$

$$
\begin{aligned}
& \left\langle n_{1} n_{2} m|\xi \eta| n_{1}+i n_{2}-i m\right\rangle \\
& \quad=\frac{1}{2} n^{2}\left\{3\left[n^{2}-\left(n_{1}-n_{2}\right)^{2}\right]+1-m^{2}\right\} \quad \text { for } i=0 \\
& \quad=2 n^{2}\left[\left(n_{1}+1\right)\left(n_{1}+1+|m|\right) n_{2}\left(n_{2}+|m|\right)\right]^{1 / 2} \quad \text { for } i=1
\end{aligned}
$$

Thus the Hamiltonian matrix for the full manifold $n, m=$ const can be written in tridiagonal form with respect to the parabolic basis of Eq. (28). A further reduction can be obtained by transforming the parabolic basis functions to functions with definite parity:

$$
\left|n_{1} n_{2} m \pm\right\rangle=2^{-1 / 2}\left\{\left|n_{1} n_{2} m\right\rangle \pm(-1)^{m}\left|n_{2} n_{1} m\right\rangle\right\}
$$

It is then easily shown that the Hamiltonian matrix in the basis of Eq. (33) 
splits into two uncoupled submatrices, one for the even parity states $\left|n_{1} n_{2} m+\right\rangle$ and one for the odd states $\left|n_{1} n_{2} m-\right\rangle$; and, from Eq. (31), the even and odd Hamiltonian matrices are tridiagonal and nearly identical. Specifically, when $n-|m|$ is even so that the even and odd matrices are of the same size, the even and odd matrices differ only in the diagonal element associated with the state for which $\left|n_{1}-n_{2}\right|=1$. When $n-m$ is odd, the Hamiltonian matrix for states with parity $(-1)^{m}$ has an additional row and column (associated with the state for which $n_{1}=n_{2}$ ); otherwise, it is identical with the matrix for states with parity $(-1)^{m+1}$. If these discrepancies in the single diagonal element (or the additional dimension) were not present, the perturbed spectrum would be doubly degenerate: each energy being associated with both an even and an odd state. This is the result of the semiclassical treatment of Goebel and Kirkman. ${ }^{(42)}$ Thus the difference between the even and odd Hamiltonians may be viewed as a result of quantum mechanical tunneling, which breaks the degeneracy of the semiclassical double-well problem. This effect of tunneling is most pronounced for orbits lying near the potential ridge $z=0$ of Figure 7, as can be seen by considering the Runge-Lenz vector. In quantum mechanics this is an operator whose $z$ component is diagonal in the basis of Eq. (28), with eigenvalues proportional to the difference $n_{2}-n_{1}$. The differences in even and odd Hamiltonian matrices are associated with states which assume the smallest possible values of $\left|n_{2}-n_{1}\right|:$ that is, those describing orbits whose major axes are most nearly perpendicular to $z$. It may also be noted that the operator $\sigma=\left(\alpha_{z}^{2}\right)^{-1 / 2} \alpha_{z}$ which gives the sign of the $z$ component of the Runge-Lenz vector $\alpha$, interchanges the even and odd states of the basis (33):

$$
\sigma\left|n_{1} n_{2} m \pm\right\rangle= \pm\left|n_{1} n_{2} m \mp\right\rangle
$$

Since this transformation almost preserves the form of the Hamiltonian matrices, $\sigma$ may be said to almost be a constant of the motion. As in the semiclassical perturbation theory, the lower-energy orbits will tend to be confined to the valleys of the potential surface around $\theta=0$ and $\pi$, i.e., $\sigma$ will tend to remain either positive or negative. As energy increases, however, the conservation of $\sigma$ ceases to hold true. Note particularly that for a planar orbit, $\alpha_{z}=0$ and so $\sigma$ is indeterminate.

Goebel and Kirkman ${ }^{(42)}$ have made considerable analysis of the solutions of the equations of degenerate perturbation theory in the parabolic basis. They note that since from Eq. (31) the equation for the eigenvector components is of the form of a three-term recursion relation, it is the finite-difference analog of a second-order differential equation. They are able to solve approximately the corresponding differential equation, in limiting cases, by a WKB approach. Moreover the recursion relations 
reduce, in some asymptotic limits, to those for standard ${ }^{(56)}$ orthogonal polynomials: the Hermite polynomials when $n \gg|m|$ and when the energy is high; Meixner polynomials for low energy and $n \gg|m|$; and Krawtchouk polynomials when $n-|m| \ll n$. In these limits oscillator strengths for transitions from the hydrogen ground state can also be computed. At the time of writing of this article, however, a detailed comparative study of these approximations has not been published.

Thus both semiclassical and quantum mechanical perturbation treatments indicate that some states become localized upon the magnetic potential ridge. It seems plausible from the discussion of planar motion given above-and it will be shown explicitly in the following section-that such states are of considerable importance in the experimental photoabsorption spectra. It may be appropriate to remark here that such localization arises quite generally in systems described by tridiagonal Hamiltonian matrices. ${ }^{(57)}$

Some simple relevant systems are a chain of coupled oscillators, or the Huckel model for the pi-electron spectrum of an aromatic molecule. If the coupling is uniform, one obtains wave functions which are entirely delocalized, and a band of energy levels in which the energy depends quadratically on the wavenumber near the bottom and the top of the band. If the coupling is nonuniform, or a substitutional impurity is introduced in the molecule, the energy spectrum is perturbed and the wave functions in the region of greatest perturbation become partially localized about the impurity. A typical energy band which arises in the perturbative treatment of the quadratic Zeeman effect can be seen in the lowest clusters of lines in Figures 9 and 10. There the energies are very nearly equally spaced at the edges of the band. States on the lower and upper edges of the band are then necessarily localized in regions of minimum and maximum potential.

NOTE (added in proof): Since the submission of this article, Herrick ${ }^{(140)}$ has published a comprehensive treatment of degenerate perturbation theory. He found that (within a given $n$ manifold) the matrix elements (32) of the diamagnetic potential are equivalent to those of the operator $U$ of Eq. (21). Therefore, in the context of perturbation theory, $U$ can be regarded as the exact diamagnetic potential [a different substitution, which omitted some terms of Eq. (21), was proposed by Labarthe $\left.{ }^{(141)}\right]$. It turns out that the resulting equations of motion can be solved in closed form in momentum space in terms of Lamé functions of Jacobian elliptic coordinates, a fact which was also noted by Soloviev. ${ }^{(139)}$

(d) Matrix Diagonalization Treatments. The many suggestive features arising in perturbative treatments have been given somewhat more concrete expression in the course of more accurate nonperturbative calculations. Evidence for the existence of a quasi-constant of the motion in the hydrogen Zeeman spectrum has been presented by Zimmerman, Kash, and 
Kleppner ${ }^{(58)}$ and by Clark and Taylor. ${ }^{(59)}$ In light of the work of Edmonds and Pullen ${ }^{(39)}$ described above, it is apparent that the validity of such a conservation law will depend on the energy of the motion, and that it must cease to apply as the ionization threshold is approached. Further development has, however, linked the quasi-constant of the motion with the asymptotic forms of the wave function on the potential ridge. ${ }^{(60)}$ It seems probable that the regularities observed in the asymptotic forms will be preserved well beyond the energy at which the approximate conservation rule fails.

The mechanics of the calculations have been given in detail elsewhere ${ }^{(45,59)}$ so only a brief summary need be provided here. Both Zimmerman, Kash, and Kleppner ${ }^{(58)}$ and Clark and Taylor ${ }^{(60)}$ diagonalized the Hamiltonian of Eq. (24) in a finite basis; Zimmerman et al. employing a basis of discrete hydrogenic wave functions and Clark and Taylor a basis of Sturmian functions. An advantage of the hydrogenic basis is that the effect of a quantum defect can be incorporated fairly readily. For the specific treatment of the hydrogen problem, however, the Sturmian basis appears preferable for a number of reasons, and we shall give it primary attention here.

A Sturmian basis was first employed in this problem by Edmonds ${ }^{(61)}$ to treat a strong field $\left(10^{4} \mathrm{kG}\right)$ case. In a general form, the Sturmian radial functions are

$$
S_{n}^{(\zeta)}(r)=\left[\frac{(n-l-1) !}{2 \cdot(n+l) !}\right]^{1 / 2} e^{-\zeta r / 2}(\zeta r)^{l+1} L_{n-l-1}^{(2 l+1)}(\zeta r)
$$

where $L$ is a Laguerre polynomial. ${ }^{(56)}$ In the diagonalization procedure these are taken in combination with spherical harmonics

$$
\psi(\mathbf{r})=\sum_{n, l} \psi_{n l} S_{n l}^{(\zeta)}(r) Y_{l m}(\hat{r})
$$

and the expansion coefficients $\psi_{n l}$ are determined. For a given $l$, the $S_{n l}$ form a complete set of functions; this is not the case with any set of discrete hydrogenic wave functions. The scale parameter $\zeta$ may take any positive value. When $\zeta=2 / n^{*}$, the radial functions $S_{n^{*} l}$ coincide, up to a normalization factor, with the hydrogen radial functions for the states with principal quantum number $n^{*}$. In Edmonds' original paper $\zeta=2$, since it was desired to examine low-lying states. The flexibility in choice of $\zeta$ allows one to "center" a calculation in a given energy region, and for computation of high Rydberg wave functions in moderate fields one employs values of $n$ * of the order of 40 . 
Another very significant advantage of the Sturmian basis is the simplicity of matrix elements. In particular, the radial matrix elements of the magnetic potential obey the selection rule $|\Delta n| \leqslant 3$. Since the angular selection rule $|\Delta l| \leqslant 2$ is also in force, the Hamiltonian matrix is very sparse, and can be written in banded form. There is a small price to be paid for achieving this sparsity, in that the $S_{n l}$ are orthogonal over a weight function equal to the Coulomb potential $1 / r$ rather than unity. Thus one has to contend with the generalized eigenvalue probem

$$
H \psi=\varepsilon B \psi
$$

where $\varepsilon$ is the energy eigenvalue and $B$ the matrix of overlap between Sturmian functions. Since the overlap between Sturmian functions vanishes when $|\Delta n|>1, B$ can always be put in tridiagonal form. Efficient algorithms exist $^{(62)}$ for the solution of Eq. (37) when, as here, $H$ and $B$ are banded matrices. On a sufficiently large computer, like the CRAY-1, determination of the eigenvalues and eigenvectors of the system (37) can be done routinely for Sturmian bases with up to 1500 elements.

When the eigenvectors have been computed, the determination of transition oscillator strengths is straightforward. Figures 9 and 10 show computed oscillator strengths for dipole transitions from the ground state of hydrogen in a field of $47 \mathrm{kG}$. The energy scale has been chosen to span the region from where perturbation theory breaks down up to a few units of cyclotron frequency below the ionization threshold. The two spectra shown would be observed in photoabsorption of light in different states of polarization. The spectrum of Figure 9 is associated with states with magnetic quantum number $m=0$, which are produced by absorption of photons polarized along the direction of the magnetic field; that of Figure 10, for which $m=1$, is generated by absorption of photons with circular polarization in the plane perpendicular to the field. Since all states have odd parity, those with $m=0$ have wave functions which vanish on the ridge $\theta=\pi / 2$, whereas the states with $m=1$ have an antinode on the ridge. Thus it should be possible to describe some of the lines in the $m=1$ spectrum in terms of the two-dimensional semiclassical solutions of Edmonds ${ }^{(35)}$ and Starace. ${ }^{(36)}$

In both spectra one sees the presence at low energy of line clusters with a well-defined shape. These can be identified with the manifolds of hydrogenic states with fixed principal quantum number $n$, and are adequately described by degenerate perturbation theory. As the energy increases the clusters begin to interpenetrate, and this simple hydrogenic description ceases to be appropriate. Note, however, that the clusters terid to pass through one another without significant perturbation of their individual members. This is more readily apparent in the $m=1$ spectrum 
than in the $m=0$ because of the monotonic distribution of oscillator strength among the lines of a given cluster. This near absence of mutual perturbation among lines with comparable oscillator strengths and nearly equal energies is the signature of a quasiconservation law. It is a complementary aspect of the curve crossings noted by Zimmerman et al. ${ }^{(58)}$ in their plot of energy levels as a function of magnetic field strength. Thus it is appropriate to regard the spectra, in this energy region, as the superposition of a number of essentially independent line series. This is analogous to the decomposition of a complicated molecular band spectrum into lines corresponding to definite rovibrational transitions, though at the moment the intuitive physical basis for such a description is not obvious. The identification of the line series in the spectrum is not ambiguous, however; in Figure 10 the first four series, in order of spectral prominence, are drawn out in separate frames. We call the set of strongest lines the first or principal series, the second, third, and fourth series following in order. Within each series one sees a regular energy spacing between members, and a decrease in oscillator strength as the energy increases. At higher energies some irregularities in the oscillator strength are apparent; these occur when members of separate series nearly coincide in energy.

The spacing between members of a series is in good agreement with the predictions of the semiclassical theory, ${ }^{(35,36)}$ as is shown in Figure 11 for the principal series. The ordinate is the radial quantum number $N$ (at low energies $N=n-2$, where $n$ is the principal quantum number) which can be regarded as a continuous function of energy in a WKB treatment. The value of $N$ to be associated with a given line of the principal series is simply determined by its position within the series. It should be noted that the absolute energies of the principal lines are very near those given by the WKB theory at high energies; thus, the total number of principal lines

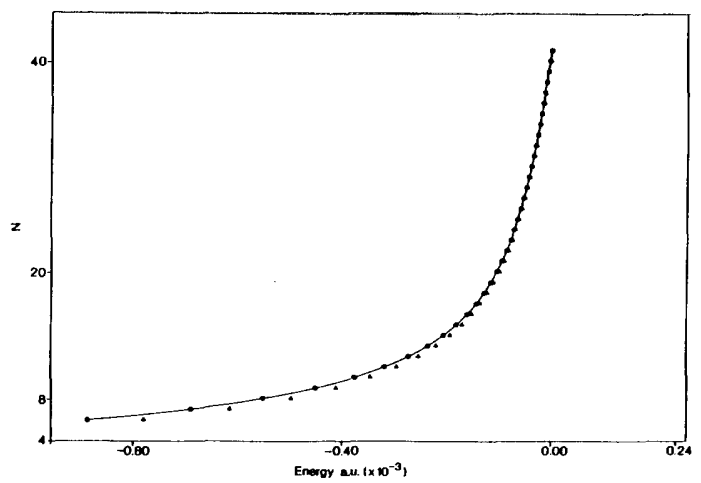

Figure 11. Comparison of the energies of the principal lines of Figure 10e with the twodimensional WKB theory of Refs. 35 and 36. Full curve: the WKB integral as a continuous function of energy; the circles are placed at points where the action is a multiple of Planck's constant. Triangles denote the positions of principal series lines. The ordinate is the number of nodes of the wave function in the plane $z=0$. (From Ref. 59.) 
in the spectrum may be expected to be close to the $N_{T}$ of Eq. (18). Note also that the oscillator strength in the principal series decreases with increasing $N$ roughly as $N^{-2.4}$.

An examination of the wave functions indicates the reason for this agreement between two-dimensional WKB and three-dimensional quantum mechanical predictions. Figure 12 shows the radial wave functions for the principal lines of Figure 10, evaluated in the plane $\theta=\pi / 2$, i.e., along the ridge of the Coulomb-magnetic potential surface of Figure 7. At small $r$ the wave functions are all nearly proportional, as would be the case in the absence of the magnetic field and as is consistent with Eq. (26). At large $r$ the wave function for each successive state of the series has an additional node. The system of functions looks very much like that which would result from a computation of the eigenfunctions of a one-dimensional Schrödinger equation. Thus in this three-dimensional calculation the confinement of the wave functions to the ridge region is seen to take place. Plots of wave functions for the higher series along the ridge show similar regularities, though not to as great a degree. In this spectrum the amount of confinement is in fact closely correlated with the magnitude of the oscillator strength.

This is seen from the variation of the wave functions in the direction across the ridge, i.e., the variation in $\theta$ with $r$ held fixed. A systematic description of the angular dependence of the wave functions at small $r$ is rather difficult when there is no predominance of a single angular momentum component, as is the case for the higher energy lines of each series. This is implied by Eq. (26), in which the amplitude associated with each angular momentum component will oscillate with increasing $r$ as a Coulomb function. In the diffraction picture of Fano as well, one must view a standing wave at small $r$ as being built up of a large number of diverging and converging trajectories. We consider now the angular behavior of the wave

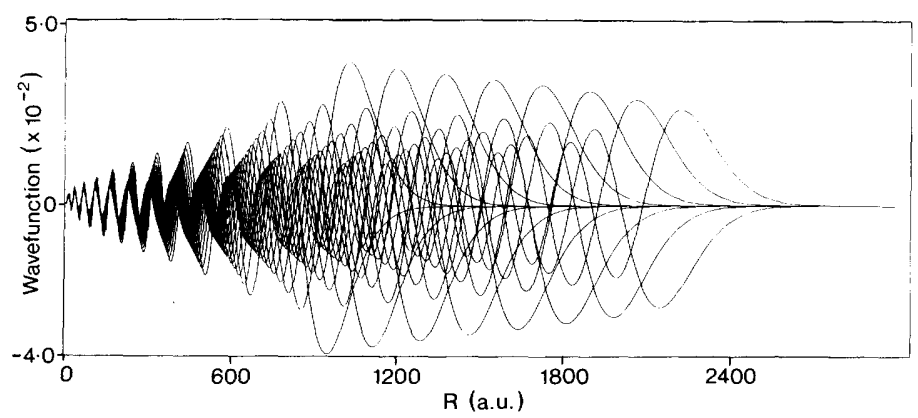

Figure 12. Wave functions for the principal series lines of Figure 10a in the plane $z=0$. (C. W. Clark and K. T. Taylor, unpublished.) 

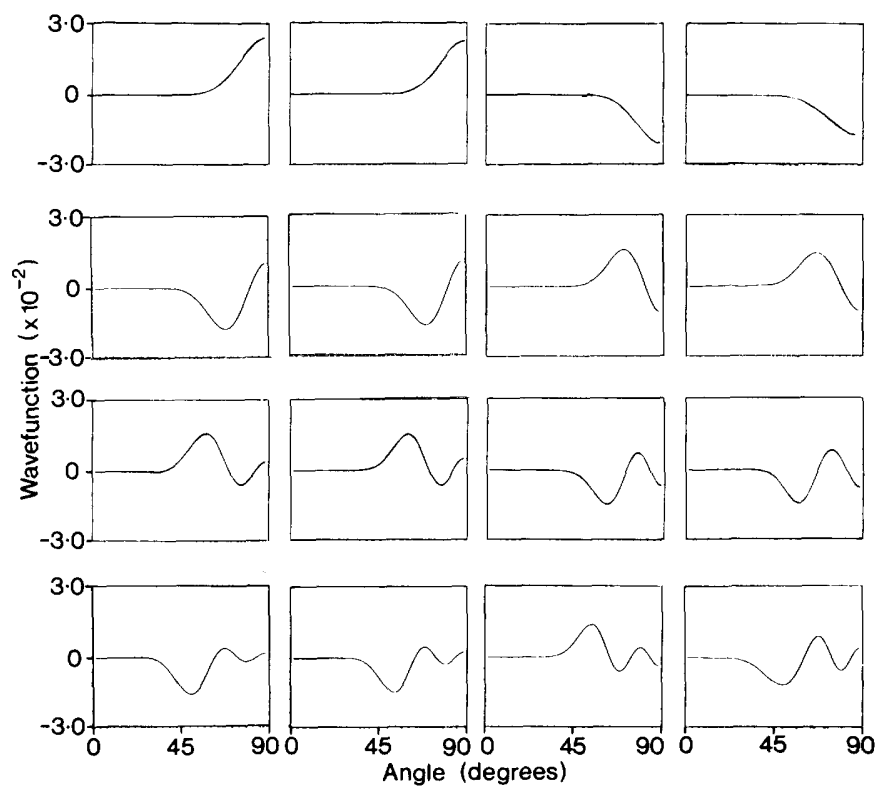

Figure 13. Wave functions along the turning surface for various states of Figure 10. First row: first, fifth, tenth, and sixteenth states of the principal series; second through fourth rows: the corresponding states of the second through fourth series, with the exception of the lower right-hand corner being the fifteenth state of the fourth series. The relationship between distance and angle is determined from Eq. (38). (From Ref. 60.)

functions at large $r$, specifically along the classical turning surface. Such a surface is the locus of the equation

$$
-1 / r+\frac{1}{2} \beta^{2} r^{2} \sin ^{2} \theta=\varepsilon
$$

where $\varepsilon$ is the energy of the state involved. These are just the various equipotential surfaces of Figure 7. Figure 13 shows wave functions for states of different series, plotted along their respective turning surfaces. The abscissa is actually the angle $\theta$, the value of $r$ to be inferred from Eq. (38) (the dependence on the azimuthal angle $\phi$ is trivial). For the states of low energy the value of $r$ is nearly constant; at higher energies the variation of $r$ with $\theta$ can be seen from Figure 7. In the top row are wave functions for the first, fifth, tenth, and sixteenth members of the principal series, as shown in Figure 10. The same order is taken for the members of the second and third series shown in the next two rows, respectively; the first, fifth, tenth, and fifteenth members of the fourth series are shown in the bottom row, the sixteenth member being strongly perturbed by a 
state from the second series (cf. Figure 10). An anticipated, the wave functions of the principal series are localized about the ridge, this behavior actually being established well before the turning surface is reached. The states of the higher series become progressively delocalized, and in a regular manner. The principal series wave functions have no nodes across the ridge; the second series wave functions have two nodes (recall that the wave functions are necessarily symmetric about $\theta=\pi / 2$ ); the third series, four nodes; and the fourth series, six nodes. States with odd numbers of nodes occur only in the $m=0$ spectrum. Thus the series number serves as an index of the degree of excitation across the ridge at large distances. However, it should be kept in mind that the motion across the ridge is intrinsically unstable, since it is always possible for the electron to fall into the valleys. Thus the results shown here are entirely at variance with any adiabatic theory; since, in a quasi-separable problem, the states of high energy which can reach the top of the ridge must also have many nodes in the valleys. It seems that this simple picture which obtains at large distances must instead be due to a particular form of interference between diverging and converging trajectories; it is more appropriate to consider these angular wave functions as a form of diffraction pattern, than as analogs to a harmonic oscillator. It is not improbable that similar phenomena will be seen in other problems involving motion about a potential ridge, for example in low-energy electron impact ionization, but this specific example does not provide enough evidence to make firm general predictions.

A physical basis for the independent series representation has been established, at least in this energy region: the members of a given series are distinguished by the degree of excitation along the potential ridge, and the series themselves are characterized by the degree of excitation across the ridge. At low energies this is a vague way of listing the properties of a quasi-constant of the motion. It remains to be seen whether such a viewpoint retains its utility as the energy increases towards, and goes beyond, the ionization limit. The calculations indicate that the members of the principal series tend to retain their form, and it seems apparent that this series will extend well into the continuum to produce the most prominent features of the photoionization spectra. The secondary features of those spectra may well be attributed to the persistence of the second, and higher, series. Since the wave functions of members of the higher series get more deeply into the valleys, it would be expected that the secondary features will be damped out more rapidly as the energy increases. This is in accordance with experimental findings. Unfortunately it is now only possible to speculate upon, rather than to review, the properties of the continuum wave functions for this problem. Their calculation does not seem feasible by any method employed thus far. 
The hydrogen atom is a system with a number of unusual properties, and many of the results reviewed here will not apply directly to the spectra of more complex atoms. For instance, the equal partitioning of oscillator strength in the clusters of the $m=0$ spectrum of Figure 9 is a result of the zero-field $l$ degeneracy, and so must be changed appreciably in the presence of a non-Coulombic core. Calculations of states with larger values of $m$, e.g., those which are involved in Balmer transitions, have revealed many near degeneracies among states of opposite parity. ${ }^{(47)}$ These must also disappear when finite quantum defects are introduced. Even a superficial examination of the spectra of a number of atoms, e.g., along the second column of the Periodic Table, reveals many significant variations of detail; almost all of which must be attributed to the changes in the atomic core.

Nevertheless there are a number of aspects of the solution to the hydrogenic problem which must have general applicability. The spacing of the quasi-Landau resonances near threshold is a universal phenomenon (albeit sometimes obscured by the motional Stark field, or by the weakness of the transition oscillator strength); and in many instances it is possible to pick out sets of lines which resemble the independent series in hydrogen. The degree to which the knowledge of these regularities can be used to build a general and practical theory remains a question for the future.

\subsubsection{Bound States in Strong Fields}

The previous sections of this review have discussed the properties of the Rydberg spectra of atomic hydrogen in high laboratory magnetic fields, particularly near the ionization threshold. In this section we review recent theoretical treatments of the ground and lowest excited levels of atomic hydrogen in magnetic fields stronger than those normally obtainable in the laboratory. Of particular interest are field strengths of the order of $10^{7}$ gauss, which is typical of white dwarf stars, and of $10^{12}$ gauss, which is typical of neutron stars. The astrophysical applications of the theory have been discussed in detail by Garstang. ${ }^{(17)}$ (Note that correspondingly large effects are found for exciton spectra in laboratory-sized magnetic fields, $10^{3} \mathrm{G} \leqslant B \leqslant 10^{5} \mathrm{G}$, due to the small effective mass of the electron. ${ }^{(63)}$ ) While we present here all recent theoretical work on hydrogenic states of low excitation in strong magnetic fields, the emphasis is on theories appropriate for magnetic fields of strength $B \leqslant 10^{9}$ gauss in which the Coulomb field is the dominant influence on the electronic motion. General aspects of atomic structure and of atomic scattering processes in extremely high magnetic fields $B \gg 10^{9}$ gauss-in which the diamagnetic interaction is the dominant influence on the electronic motion-are discussed in Section 6 . 
(a) Quasiseparation of Electronic Motion Using an Adiabatic Approximation. The Hamiltonian in Eq. (9) is difficult to treat since the effective potential is not separable. The only good quantum numbers are parity and the axial component of orbital angular momentum $m$. Equation (9) may, however, be cast into a form susceptible to an adiabatic separation of variables. ${ }^{(14,64,65)}$ To this end we write the wave function $\psi(\mathbf{r})$ for the reduced mass particle as

$$
\psi(\mathbf{r})=\frac{\chi_{m}(r, \theta)}{r} \frac{e^{i m \phi}}{(2 \pi)^{1 / 2}}
$$

and substitute Eq. (39) in Eq. (9). We obtain in atomic units (i.e., $m=e=$ $\hbar=1)$ the following equation for $\chi_{m}(r, \theta)$ :

$$
\left[\frac{1}{\mu} \frac{d^{2}}{d r^{2}}+\frac{2}{r}+2 E^{\prime}-\frac{\Lambda_{m}\left(\alpha r^{2}, \theta\right)}{\mu r^{2}}\right] \chi_{m}(r, \theta)=0
$$

where

$$
\Lambda_{m}\left(\alpha r^{2}, \theta\right) \equiv-\frac{1}{\sin \theta} \frac{\partial}{\partial \theta}\left(\sin \theta \frac{\partial}{\partial \theta}\right)+\frac{m^{2}}{\sin ^{2} \theta}+\alpha^{2} r^{4} \sin ^{2} \theta
$$

In Eqs. (40) and (41) $\alpha$ is a strength parameter defined as

$$
\alpha \equiv \frac{B}{2 c}=\left(2.127 \times 10^{-10} \text { a.u. } / \mathrm{G}\right) B(\mathrm{G})
$$

and in Eq. (40) the prime on the energy $E^{\prime}$ indicates that the linear Zeeman energy is subtracted from the total energy $E$, i.e.,

$$
E^{\prime} \equiv E-\alpha m(g / \mu)
$$

When the operator $\Lambda_{m}\left(\alpha r^{2}, \theta\right)$ defined in Eq. (41) is considered to depend only parametrically on the quantity $\alpha r^{2}$, it has as eigenstates the oblate spheroidal angle functions $g_{m \nu}\left(\alpha r^{2}, \theta\right)$ :

$$
\Lambda_{m}\left(\alpha r^{2}, \theta\right) g_{m \nu}\left(\alpha r^{2}, \theta\right)=U_{m \nu}\left(\alpha r^{2}\right) g_{m \nu}\left(\alpha r^{2}, \theta\right)
$$

where $\nu$ labels the eigenstates and $U_{m \nu}\left(\alpha r^{2}\right)$ are the corresponding eigenvalues. In the limit that $\alpha r^{2} \rightarrow 0$ (due either to $B \rightarrow 0$ or $r \rightarrow 0$ ), the index $\nu$ becomes the ordinary orbital angular momentum quantum number $l$, $U_{m \nu}\left(\alpha r^{2}\right)$ becomes $l(l+1)$, and $g_{m \nu}$ becomes the associated Legendre polynomial $P_{l}^{m}(\cos \theta)$. For finite values of the parameter $\alpha r^{2}$, each oblate 
spheroidal angle function may be written as a linear combination of associated Legendre polynomials. More specifically, as $\alpha r^{2}$ increases from zero, each oblate spheroidal angle function with index $\nu$ loses its identity to the single associated Legendre polynomial with $l=\nu$ and must be represented by an increasingly larger sum over Legendre polynomials with other orbital angular momenta $l \neq \nu$ and the same parity. Thus the oblate spheroidal angle functions include implicitly a large amount of magnetic field distortion of the atom's spherical symmetry which spectroscopically is observed as "l-mixing." Due to the parametric dependence of the oblate spheroidal angle functions on the radial coordinate, they do not represent eigenstates for the angular part of the wave function $\chi_{m}(r, \theta)$ in Eq. (40). However, in an adiabatic approximation this is a good first approximation in many cases, as shown below.

In an exact treatment, the wave function $\chi_{m}(r, \theta)$ must be expanded as a linear combination of oblate spheroidal angle functions with radially dependent coefficients:

$$
\chi_{m}(r, \theta)=\sum_{\nu^{\prime}}^{\prime} h_{m \nu^{\prime}}(r) g_{m \nu^{\prime}}\left(\alpha r^{2}, \theta\right)
$$

The prime on the summation indicates that either even or odd $\nu \geqslant|m|$ are included in the summation depending on whether the party of the state is even or odd. Substituting Eq. (45) into Eq. (40), multiplying from the left by $g_{m \nu}$, and integrating over $\theta$ gives the following set of coupled differential equations for the radial function $h_{m \nu}(r)^{(64)}$ :

$$
\begin{aligned}
{\left[\frac{1}{\mu} \frac{d^{2}}{d r^{2}}\right.} & \left.+\frac{2}{r}+2 E^{\prime}-\frac{U_{m \nu}\left(\alpha r^{2}\right)}{\mu r^{2}}\right] h_{m \nu}(r) \\
& +2 \sum_{\nu^{\prime}}\left(g_{m \nu}, \frac{\partial g_{m \nu^{\prime}}}{\partial r}\right) \frac{d}{d r} h_{m \nu^{\prime}}(r) \\
& +\sum_{\nu^{\prime}}\left(g_{m \nu}, \frac{\partial^{2} g_{m \nu^{\prime}}}{\partial r^{2}}\right) h_{m \nu^{\prime}}(r)=0
\end{aligned}
$$

where

$$
\left(g_{m \nu}, \frac{\partial^{n} g_{m \nu^{\prime}}}{\partial r^{n}}\right) \equiv \int_{0}^{\pi} g_{m \nu}\left(\alpha r^{2}, \theta\right) \frac{\partial^{n}}{\partial r^{n}} g_{m \nu}\left(\alpha r^{2}, \theta\right) \sin \theta d \theta
$$

Whether or not an adiabatic approximation is reasonable depends on the strength of the first and second derivative coupling terms in Eq. (46). Use of the Hellman-Feynman theorem ${ }^{(66,65)}$ shows that they are large only near 
avoided crossings in the "potential" curves $U_{m \nu}\left(\alpha r^{2}\right)$. These curves are shown for the odd $\nu$-values 1, 3, 5, 7, and 9 in Figure 14. In general, the locus of avoided crossings between these curves lies along the curve defined by $U_{m \nu}\left(\alpha r^{2}\right)=m^{2}+\alpha^{2} r^{4}$ as this defines the classical turning point for the oblate spheroidal angle functions for motion in $r$ along $\theta=90^{\circ}$ [cf. Eqs. (41) and (44)]. That is, at any larger value of $\alpha^{2} r^{4}$ the "kinetic energy" of the oblate spheroidal angle functions, given by the first term on the right in Eq. (41), becomes negative since the potential energy, $m^{2}+\alpha^{2} r^{4}$, on the right of Eq. (41) for $\theta=90^{\circ}$ becomes greater than the eigenvalue $U_{m \nu}$ of $\Lambda_{m}$ [cf. Eq. (44)]. Hence tunneling behavior must set in-unless, that is, a transition is made to a higher state $\nu$ with a larger $U_{m \nu}$, in which case the particle can propagate outward in $\alpha r^{2}$ until it reaches the turning point of the new potential.

Examination of Figure 14 shows that the first avoided crossing, that between the curves $\nu=1$ and $\nu=3$, occurs near $\alpha r^{2}=3$. For a magnetic field $B$ of order $10^{5} \mathrm{G}$, which is a typical strength for laboratory magnetic fields, this corresponds to an $r$ value of $375 \mathrm{bohr}$; for $B$ of order $10^{7} \mathrm{G}$, this corresponds to an $r$ value of $37.5 \mathrm{bohr}$; and for $B$ of order $10^{9} \mathrm{G}$, this corresponds to an $r$ value of $3.75 \mathrm{bohr}$. Thus for hydrogenic states having a radial extent less than these values of $r$, one may quite accurately represent the wave function for the state in terms of a single oblate spheroidal angle function,

$$
\chi_{m \nu}(r, \theta) \approx h_{m \nu}(r) g_{m \nu}\left(\alpha r^{2}, \theta\right)
$$

where $h_{m \nu}(r)$ is obtained by solving Eq. (46) in the adiabatic approximation in which the coupling terms are set equal to zero.

Such adiabatic calculations have been carried out by Starace and Webster ${ }^{(64)}$ for the $1 s, 2 s$, and $2 p$ levels of atomic hydrogen. The calculated energies-which may be shown to be rigorous lower bounds on the true

Figure 14. Oblate spheroidal eigenvalues $U_{m \nu}\left(\alpha r^{2}\right)$ for $m=1$ and $\nu=1,3,5,7$, and 9 plotted as a function of the parameter $\alpha r^{2}$.

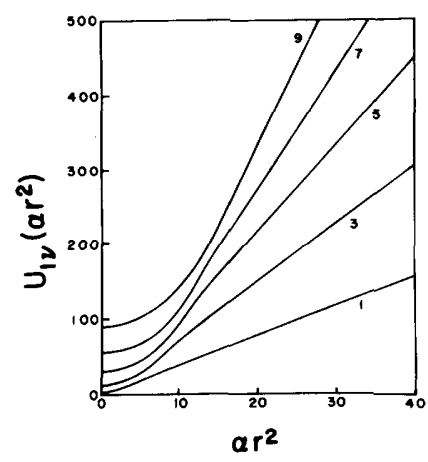


energy for the lowest states of each symmetry-are shown in Figure 15 for the $1 s$ and $2 p$ levels and compared with the best available variational upper bounds on these same energies. ${ }^{(67)}$ A detailed comparison of the calculated binding energy of the $1 s$ level with other theoretical results is shown in Table 1. The adiabatic oblate spheroidal angle function treatment gives results that agree very well with more elaborate calculations for magnetic fields up to about $10^{9} \mathrm{G}$.

Even when an adiabatic approximation is not valid, the oblate spheroidal angle function representation may be useful. The reason is that the eigenvalue curves in Figure 14 indicate how many oblate spheroidal adiabatic functions need to be coupled at any value of the parameter $\alpha r^{2}$. Of course, due to the variation of the locus of avoided crossings with the square of the parameter $\alpha r^{2}$, the number of adiabatic solutions which must be coupled increases rapidly with increasing $r$ but less rapidly with increasing magnetic field strength $B$. Thus the method appears most useful for treating the lowest levels of an atom, even in high fields for which nonadiabatic couplings must be considered, rather than for treating Rydberg states in high magnetic fields due to their large spatial extensions in $r$. This state of affairs is not peculiar to the oblate spheroidal representation, but is a feature of any theoretical representation based upon spherical symmetry. All such theoretical methods have difficulty representing the cylindrically symmetric magnetic field distortion of the electronic orbit far from the nucleus of the atom.

We should mention that a number of other adiabatic treatments have been given for atomic hydrogen in a uniform magnetic field. ${ }^{(68-73)}$ All of these, however, employ cylindrical coordinates, thus applying only to very high field strengths $B>10^{9} \mathrm{G}$. Furthermore these methods give a poor description of the electron wave function in the neighborhood of the origin, where optical absorption takes place. An attractive feature of the adiabatic approach in spherical coordinates presented above is that the electron wave

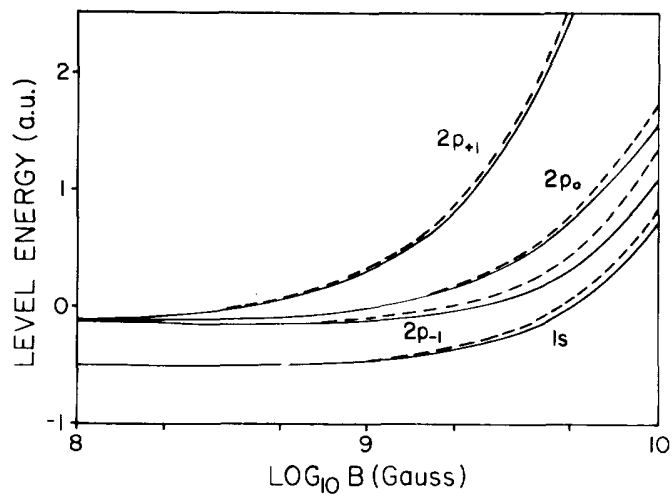

Figure 15. Upper and lower bounds for the hydrogenic $1 s$ and $2 p$ level energies in a uniform magnetic field. Solid lines: adiabatic results of the oblate spheroidal treatment of Starace and Webster (Ref. 64); dashed lines, best variational upper-bound results (cf. Ref. 64, Tables IIIV). 


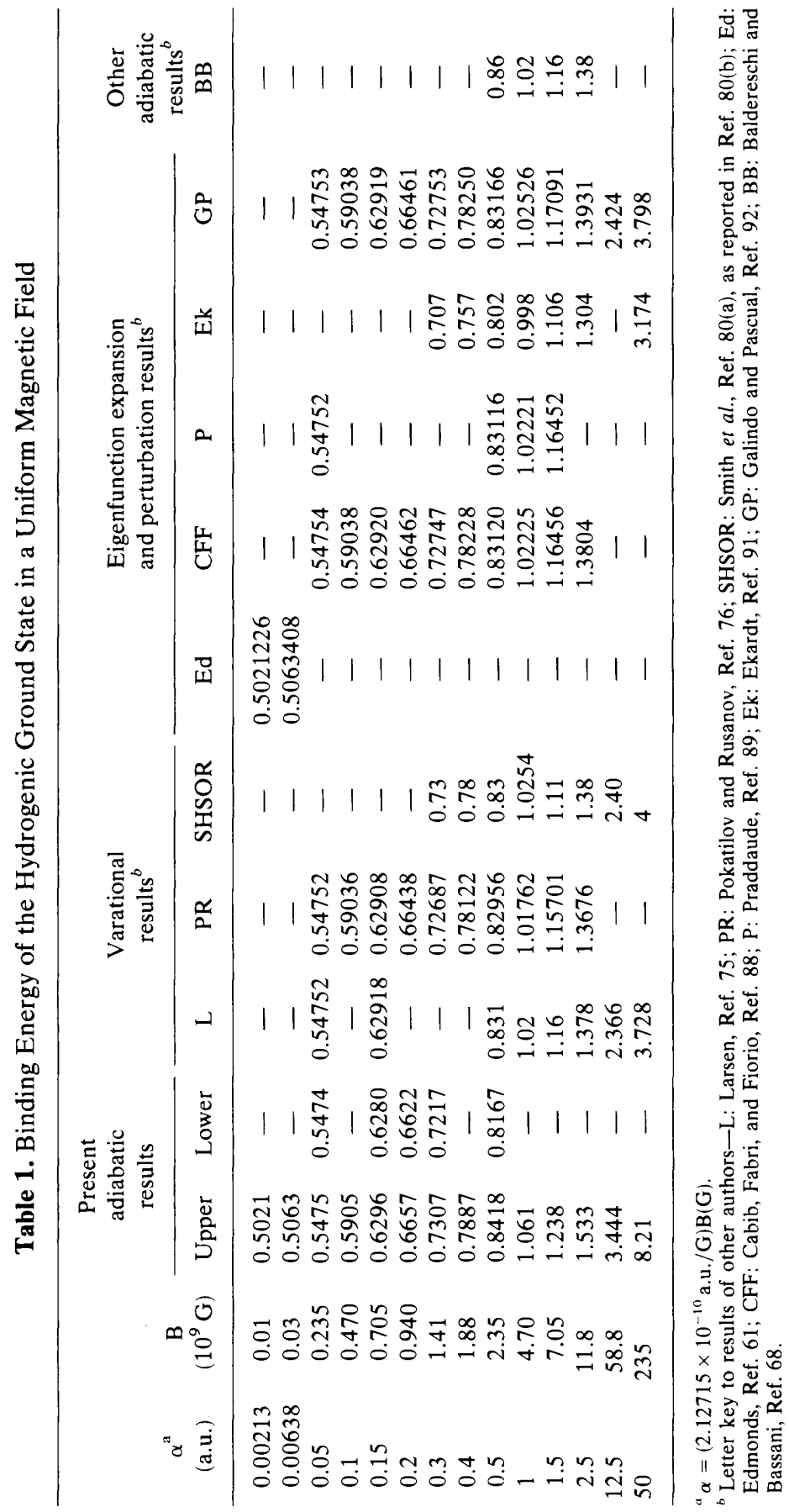


function is accurately represented near the origin no matter how high the field strength. However, the matching of the spherically symmetric wave function near the origin onto wave functions having the cylindrical symmetry more appropriate far from the origin remains an unsolved theoretical problem.

(b) Other Recent Theoretical Developments. Besides the adiabatic approximations, numerous studies for atomic hydrogen in a uniform magnetic field have been carried out using either variational ${ }^{(68,74-87)}$ or eigenfunction expansion ${ }^{(59,61,69,88-92)}$ methods. Variational methods, of course, are most useful for calculating upper bounds on the energy of the lowest state of a given symmetry. Upper bounds on excited energy levels may also be obtained by variational methods, but only at the cost of greater computational labor. ${ }^{(93)}$ Eigenfunction expansion calculations carry out an exact or approximate diagonalization of the Hamiltonian, but often require the use of very large numbers of basis states to achieve accurate results.

Very recently attention has been focused on mathematically rigorous perturbative treatments of atomic hydrogen in a uniform magnetic field. Killingbeck ${ }^{(48)}$ has separated the quadratic Zeeman term in the Hamiltonian into so-called $s$ and $d$ components, as follows:

$$
\frac{e^{2} B^{2}}{8 \mu c^{2}}\left(x^{2}+y^{2}\right)=\left(\frac{e^{2} B^{2}}{8 \mu c^{2}}\right)\left[\frac{2}{3} r^{2} P_{0}(\cos \theta)-\frac{2}{3} r^{2} P_{2}(\cos \theta)\right]
$$

Here $P_{0}(\cos \theta)$ and $P_{2}(\cos \theta)$ are Legendre polynomials: $P_{0}=1$ and $P_{2}=$ $\left(\frac{3}{2} \cos ^{2} \theta-1 / 2\right)$. Initially Killingbeck drops the second term in Eq. (49) and solves the remaining Hamiltonian exactly; since $P_{0}(\cos \theta)=1$ the potential has only radial dependence. The resulting energies are rigorous upper bounds on the correct energies and in fact turn out to be very accurate for magnetic fields less than $10^{9} \mathrm{G}$. Treating then the second term in Eq. (49) perturbatively, Killingbeck is able to obtain rigorous upper and lower bounds on the $1 s$ level of hydrogen which agree to four digits at $10^{9} \mathrm{G}$ !

Asymptotic perturbation expansion formulas for the ground-state energy of atomic hydrogen in a uniform magnetic field have been studied by Avron and co-workers. ${ }^{(49,94,95)}$ In one such study, Avron et al. ${ }^{(49)}$ used the Bender-Wu formulas ${ }^{(95)}$ to obtain analytic expressions (to order $1 / n$ ) for the coefficients $E_{n}$ in the following expansion for the ground-state energy:

$$
E(B)=\sum_{n=0}^{\infty} E_{n}\left(\frac{B^{2}}{8}\right)^{n}
$$

Their result is

$$
E_{n}=(-1)^{n+1}\left(\frac{4}{\pi}\right)^{5 / 2}\left(\frac{8}{\pi^{2}}\right)^{n}\left(2 n+\frac{1}{2}\right) !\left[1+O\left(\frac{1}{n}\right)\right]
$$


In another study, Avron, Herbst, and Simon ${ }^{(94)}$ obtain a detailed asymptotic expression for the ground-state binding energy of hydrogen in an extremely large magnetic field. It has been known for some time now that in extremely strong magnetic fields the hydrogenic electron is forced so close to the nucleus that its binding energy increases as $\ln ^{2} B .{ }^{(70,77,96)}$ Avron et al. ${ }^{(49)}$ obtain instead a new implicit asymptotic formula for the binding energy whose numerical predictions are in excellent agreement with earlier work.

\section{Stark Effect in the Hydrogen Atom}

The standard treatment of the Stark effect in the hydrogen atom is in parabolic coordinates. ${ }^{(26,97)}$ The Schrödinger equation for an electron in a Coulomb and a uniform electric field is separable into one-dimensional WKB-type equations in the $\xi$ and $\eta$ coordinates. Various techniques have been developed for calculating the Stark effect for atoms of low excitation. ${ }^{(98-104)}$ Recent review articles ${ }^{(18-20)}$ have covered these works. Renewed interest has been generated by the recent experimental observations of regular intensity modulations in the photoionization cross section of atoms in an external electric field near and above the zero-field ionization limit. ${ }^{(12,105-107)}$ The polarization dependence of these features has been discussed $^{(108)}$ and confirmed by a numerical calculation of the Stark photoionization process in hydrogen by Luc-Koenig and Bachelier, ${ }^{(109)}$ who have adapted a method developed by Blossey ${ }^{(110)}$ for Wannier excitons. Semianalytic theories for this effect have also been developed. ${ }^{(111,112)}$ Below we review this recent work on photoionization of atoms in a uniform electric field. Recent advances in high-order perturbative calculations will be briefly discussed. ${ }^{(1}$

\subsection{Semiclassical Treatments: Photoionization Cross Section}

Following Landau and Lifshitz, ${ }^{(97)}$ the Schrödinger equation for combined Coulomb and Stark potentials, $-e^{2} / r+e \mathbf{F} \cdot \mathbf{r}$, is separable in parabolic coordinates $\xi=r+z, \eta=r-z$, and $\phi=\tan ^{-1}(y / x)$. The two separated one-dimensional equations for $\xi$ and $\eta$ are

$$
\begin{aligned}
& \frac{d^{2} \chi_{1}(\xi)}{d \xi^{2}}+\left[\frac{1}{2} \varepsilon-2\left(\frac{m^{2}-1}{8 \xi^{2}}-\frac{\beta_{1}}{2 \xi}+\frac{F}{8} \xi\right)\right] \chi_{1}(\xi)=0 \\
& \frac{d^{2} \chi_{2}(\eta)}{d \eta^{2}}+\left[\frac{1}{2} \varepsilon-2\left(\frac{m^{2}-1}{8 \eta^{2}}-\frac{\beta_{2}}{2 \eta}-\frac{F}{8} \eta\right)\right] \chi_{2}(\eta)=0
\end{aligned}
$$

and the separation parameters $\beta_{1}$ and $\beta_{2}$ satisfy $\beta_{1}+\beta_{2}=1$. The energy 
$\varepsilon$ is regarded as a parameter which has a definite value, and the $\beta_{1}$ and $\beta_{2}$ as eigenvalues of corresponding equations. These quantities are determined by solving Eqs. (51) and (52) as functions of $\varepsilon$ and field strength $F$. The condition $\beta_{1}+\beta_{2}=1$ then gives the required relation between $\varepsilon$ and $F$, i.e., the energy as a function of the external field $F$. The eigenstate wave function is represented by

$$
\left|\varepsilon, F ; n_{1} m\right\rangle=N_{\varepsilon n_{1}} \chi_{1}(\xi) \chi_{2}(\eta) e^{i m \phi}(2 \pi)^{-1 / 2}
$$

where $n_{1}$ is the parabolic quantum number representing the number of nodes in the $\xi$-mode and $m=0, \pm 1, \pm 2 \ldots$ The $\chi_{1}(\xi)$ and $\chi_{2}(\eta)$ are Coulombic for small values of $\xi$ and $\eta$, and are normalized to $\xi^{m / 2}$ and $\eta^{m / 2}$ as $(\xi, \eta) \rightarrow 0$. The total wave function is normalized by $N_{\varepsilon n_{1}}$ to satisfy the orthonormality condition.

For low excited states and moderate electric field strengths, one can use a perturbation method to evaluate the Stark shifts. ${ }^{(102,104)}$ When the electron energy increases, the binding energy of the electron can be comparable with the Stark energy shift. Also, the electron can ionize due to the quantum tunneling created by the Stark field. Figure 16 shows the potential energy in Eq. (51) and (52)

$$
U_{1}(\xi)=\frac{m^{2}-1}{8 \xi^{2}}-\frac{\beta_{1}}{2 \xi}+\frac{F}{8} \xi \quad \text { and } \quad U_{2}(\eta)=\frac{m^{2}-1}{8 \eta^{2}}-\frac{\beta_{2}}{2 \eta}-\frac{F}{8} \eta
$$

The dependence of $\beta_{1}$ and $\beta_{2}$ on energy $\varepsilon$ are fixed by the Bohr-Sommerfeld
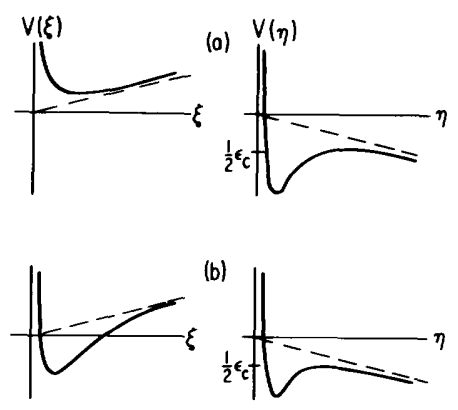

Figure 16. Qualitative plots of the potentials $U_{1}(\xi)$ [denoted here by $V(\xi)]$ and $U_{2}(\eta)$ [denoted here by $V(\eta)]$ in Eqs. (51) and (52) for $m>1, F \geqslant 0$, and sample values of $\beta_{1}=1-\beta_{2}$ : (a) $\beta_{1} \approx-0.1$,

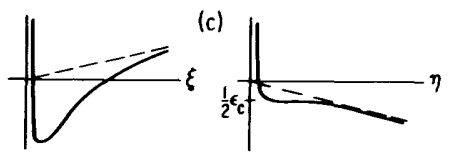
(b) $\beta_{1} \approx 0.4$, and (c) $\beta_{1} \approx 0.9$. The dotted lines represent the Stark potentials $\frac{1}{4} F \xi$ and $-\frac{1}{4} F \eta$. The top of the potential hump in $\eta, \frac{1}{2} \varepsilon_{c}$, and the potential coalesce in (c) where $\beta_{2} \approx \beta_{\text {crit }} \sim 0.1$. (From Ref. 112.) 
quantization rule

$$
\begin{aligned}
& \int_{\xi_{1}}^{\xi_{2}}\left\{2\left[\frac{1}{4} E-U_{1}(\xi)\right]\right\}^{1 / 2} d \xi=\left(n_{1}+\frac{1}{2}\right) \pi \\
& \int_{\eta_{1}}^{\eta_{2}}\left\{2\left[\frac{1}{4} E-U_{2}(\eta)\right]\right\}^{1 / 2} d \eta=\left(n_{2}+\frac{1}{2}\right) \pi
\end{aligned}
$$

where $n_{1}$ and $n_{2}$ are parabolic quantum numbers. These integrals can be solved numerically. For a given energy level $E_{n}$, according to the classical picture, there is a critical electric field $F_{c}$, ${ }^{(26)}$ where $F_{c}=E^{2} / 4 \beta_{2}=$ $1 / 16 n^{4} \beta_{2}$, such that ionization can take place only if $E_{n}$ exceeds this value. However, in reality, ionization can take place for field strengths lower than this because of the quantum mechanical tunneling. One can see this effect readily by examining the potentials in Figure 16. The potential $U_{1}(\xi)$ increases as $\xi \rightarrow \infty$, so that the eigenvalues $\beta_{1}$ correspond to a bound mode and $\chi_{1}(\xi)$ decays exponentially. In contrast, there is a potential barrier in the $\eta$-mode and the wave function $\chi_{2}(\eta)$ is oscillating at large $\eta$. Thus, the $\eta$-mode corresponds to ionization and tunneling. The asymptotic expression for $\chi_{2}(\eta)$ can be obtained by combining an independent pair of Airy functions at large $\eta^{(103,112)}$ :

$$
\chi_{2}(\eta)=A_{n_{1}, m}(\varepsilon, F)\left[\frac{2}{\pi k(\eta)}\right]^{1 / 2} \sin \left\{\left[\Delta(\eta)+\frac{1}{4} \pi\right]+\delta_{n_{1} m}(\varepsilon, F)\right\}
$$

Here $\delta_{n_{1} m}(\varepsilon, F)$ is the total phase accumulated over the interval $\eta=0$ to $\eta=\infty$ and $A_{n_{1} m}(\varepsilon, F)$ is the asymptotic amplitude and $\Delta(\eta)$ is the WKB phase integral. An energy level of the atom to which an electron is excited in the presence of a Stark field will reveal itself with a finite width corresponding to an ionization yield. This decay of atomic resonances due to the Stark effect involves two coupled motions, the bounded $\xi$-mode and the continuum $\eta$-mode, with a coupling through the separation parameter, $\beta_{1}+\beta_{2}=1$. It thus resembles the familiar picture of autoionization. ${ }^{(108)}$ The amplitude $A$ and phase shift $\delta$ can be used to fit a simple Breit-Wigner formula for the Stark resonance, which can be characterized by a resonance position $\varepsilon_{n_{2}}$ and a width $\Gamma_{n_{2}}{ }^{(103,108,112)}$

$$
\delta_{n_{1} m}(\varepsilon, F)=\delta_{n_{1} m}\left(\varepsilon_{n_{2}}, F\right)+\tan ^{-1}\left[\Gamma_{n_{2}} / 2\left(\varepsilon-\varepsilon_{n_{2}}\right)\right]
$$

and

$$
A_{n_{1} m}^{2}(\varepsilon, F)=A_{n_{1} m}^{2}\left(\varepsilon_{n_{2}}, F\right)\left[\left(\varepsilon-\varepsilon_{n_{2}}\right)^{2}+\frac{1}{4} \Gamma_{n_{2}}^{2}\right]
$$


The width $\Gamma_{n_{2}}$ is related to the ionization rate, and calculations of these rates in hydrogen in the vicinity of $E_{c}{ }^{(102-104)}$ as well as near and above the zero-field limit ${ }^{(109,112)}$ are available.

The photoionization cross section of the hydrogen atom can be obtained easily by replacing the energy-normalized wave function $\left|\varepsilon, F ; n_{1} m\right\rangle$ in Eq. (53) by the zero-field hydrogen wave function $\left|\varepsilon, 0 ; n_{1} m\right\rangle,{ }^{(112)}$ since the ground-state wave function $|g\rangle$ is concentrated near the nucleus, where the Stark field is negligible. The usual selection rules apply: (1) $\Delta m=0$ for light polarization parallel to the field, and (2) $\Delta m= \pm 1$ for perpendicular light polarization. The photoionization cross section is ${ }^{(111,112)}$

$$
\sigma_{F}^{m}(\varepsilon)=\left(\frac{4 \pi^{2}}{137} \hbar \omega\right) d_{l m}^{2}(\varepsilon) \sum_{n_{1}=0}^{\infty} N_{\varepsilon n_{1} m}^{2}(\varepsilon) \alpha_{l m}^{2}\left(\beta_{1}, \nu\right)=\sum_{n_{1}} \sigma_{F, n_{1}}^{m}(\varepsilon)
$$

where $\hbar \omega=E_{i}-E_{f}$ and $\nu=(-\varepsilon / 13.6 \mathrm{eV})^{1 / 2}$. The magnitude of the photoionization cross section depends on three factors: (1) the radial dipole integral

$$
d_{l m}(\varepsilon)=\int_{0}^{\infty} d r F_{l}^{*}(\varepsilon, r) r_{m} R(r)
$$

where $R(r)$ and $F_{l}(\varepsilon, r)$ are wave functions of the ground state and final state, respectively, and where $r_{m}$ equals $z$ for $m=0$ and $\sqrt{\frac{1}{2}}(x \pm i y)$ for $m= \pm 1$; (2) the normalization factor of Eq. (53), $N_{\varepsilon n_{1} m}^{2}(\varepsilon)$; and (3) the geometric factor $\alpha_{l m}\left(\beta_{1}, \nu\right)$, which represents the projection of the confluent hypergeometric factors of $\left|n_{1}, m\right\rangle$ with $F=0$, onto the associated Legendre polynomials $P_{l}^{m}(\cos \phi) .^{(116,117)}$ The dipole factor $d_{l m}(\varepsilon)$ can be obtained from the intensities of zero-field spectral lines.

The polarization dependence of photoionization cross sections have been carried out ${ }^{(118)}$ and the results are shown in Figure 17. They are in good agreement with exact numerical calculations by Luc-Koenig and Bachelier ${ }^{(109)}$ and with the early findings of the intensity modulations near the zero-field ionization limit for $\pi$-polarization (i.e., the $m=0$ mode). ${ }^{(12)}$ To understand this effect, it is more transparent to transform the eigenstate for a given energy from parabolic coordinates to spherical polar coordinates

$$
\left|\varepsilon, n_{1} m\right\rangle=N_{n_{1} m}(\varepsilon) \sum_{l} F_{l}(r) Y_{l m}(\theta, \phi) \alpha_{l m}\left(\beta_{1}, \nu\right)
$$

The spherical harmonics $Y_{l m}(\theta, \phi)$ are nonzero for $\theta=0^{\circ}$ only for the $m=0$ component of any arbitrary $l$. This condition can be fulfilled provided 

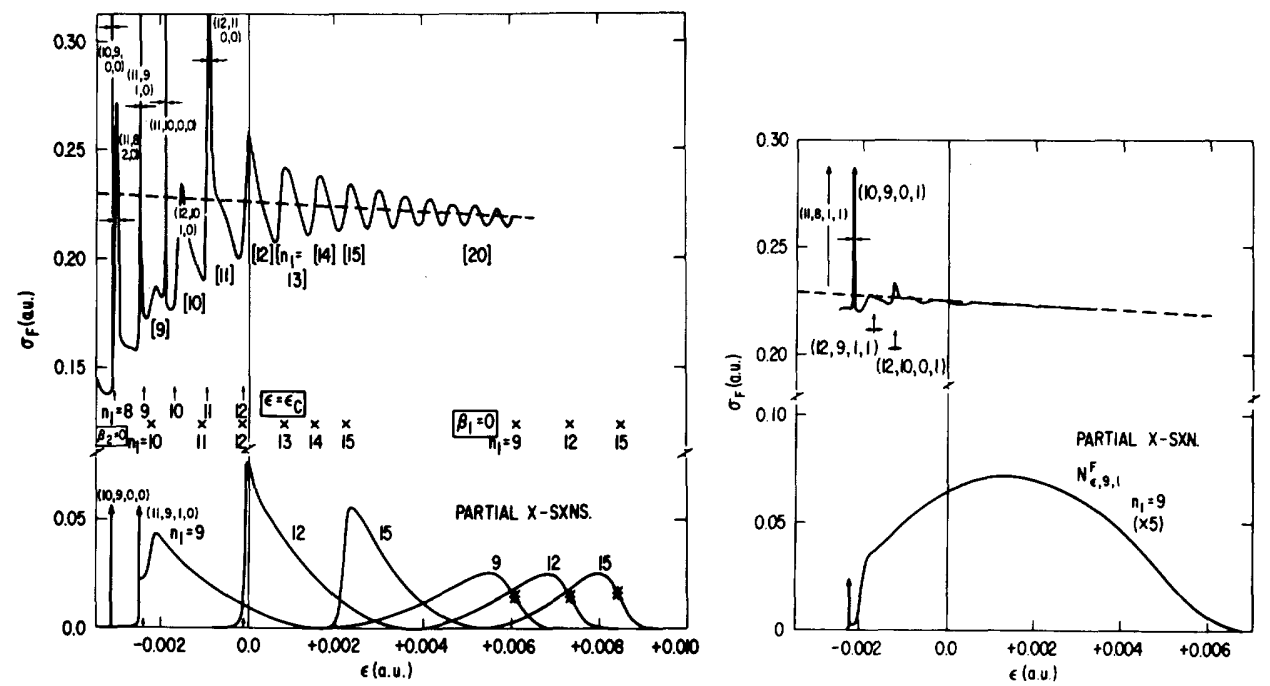

Figure 17. Photoionization cross section (in a.u.) of the ground state of atomic hydrogen in a Stark field $F=77 \mathrm{kV} / \mathrm{cm}$ vs. energy $\varepsilon$ (in a.u.) for different light polarizations. (a) The upper part shows the total cross section from Eq. (56) for $\pi$-polarization. The dashed curve indicates $\sigma_{0}(\varepsilon)$ for $F=0$. The lower part shows the partial cross sections. (b) The total cross section for $\sigma$-polarization. The parabolic quantum numbers $\left(n, n_{1}, n_{2}, m\right)$ for $\varepsilon<0$ and the $n_{1}$-channel corresponding to the peaks for $\varepsilon \geqslant 0$ are marked in the figure. (Courtesy of D. A. Harmin.)

that the electron's energy is above the potential barrier created by the Coulomb and Stark field. The barrier will attain its maximum for $\theta=0^{\circ}$. However, only that portion of the electron's motion parallel to the field axis, i.e., the $z$ axis, and having $m=0$, has a high probability of being scattered by the infinite barrier toward the core and thus gains intensity by overlapping extensively with the ground-state wave function. For a given $n_{1}$ manifold, the $m=0$ mode corresponds then to the electron's motion being confined and the charge distribution being stretched along the field axis. Indeed, one can understand this by examining the results shown in Figure 17 in parabolic coordinates. Each intensity maximum (or resonance) for energy $\varepsilon>0$ in the photoionization cross section corresponds to the energy for a given $n_{1}$ manifold in the partial cross section such that $\beta_{1} \sim 1$ and $\beta_{2} \sim 0$. The value $\beta_{1} \sim 1$ corresponds to the maximum distribution of the excited wave function along the $z$ axis, and this effect is most enhanced for final states with $m=0$. When the electron's energy is below the potential barrier, $\varepsilon<0$, its motion is more conditioned by the barrier penetration effect. In other words, the condition to produce $m=0$ intensity modulation will not be so well satisfied. Photoionization calculations from the unperturbed $3 p$ state in hydrogen show pronounced intensity modulations, in 

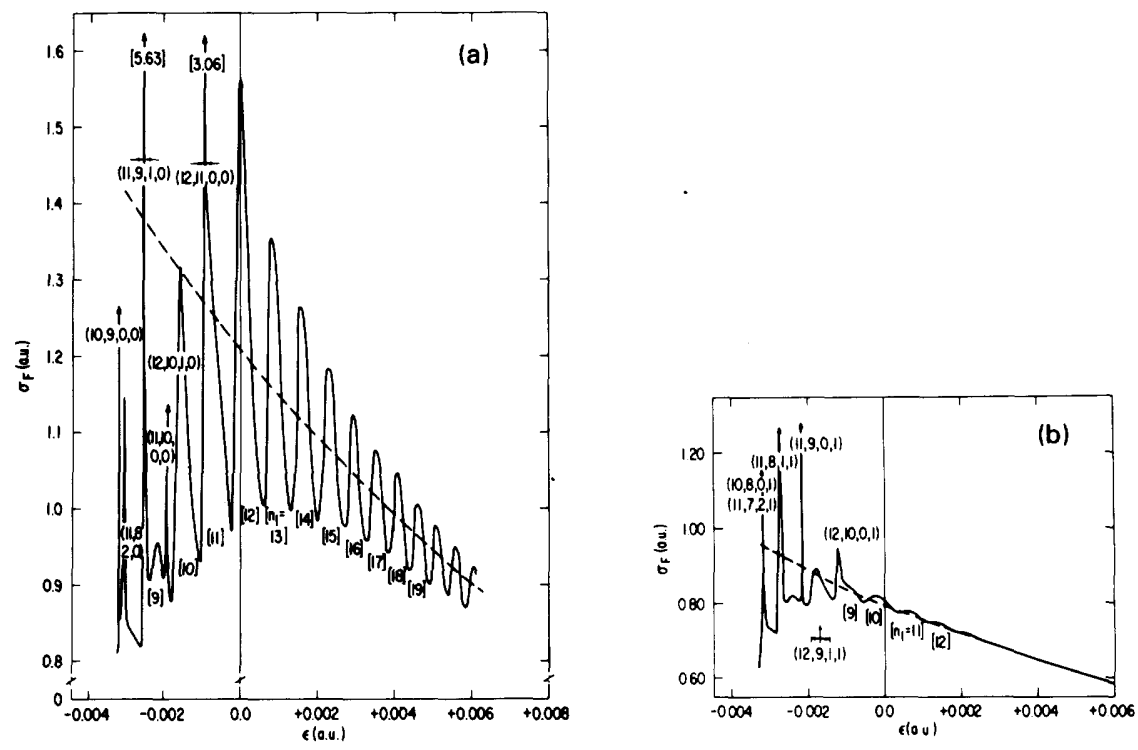

Figure 18. Photoionization cross sections (in a.u.) of the $3 p$ state of atomic hydrogen in a Stark field $F=77 \mathrm{kV} / \mathrm{cm}$ vs. energy $\varepsilon$ (in a.u.) for different light polarizations. (a) $\pi \pi$ : Transition from $3 p, m=0$ to $\varepsilon s+\varepsilon d, m=0$. (b) $\sigma^{ \pm} \pi$ (or $\pi \sigma^{ \pm}$): Transition from $3 p$, $m=1$ or 0 to $\varepsilon d, m=1$. (Courtesy of D. A. Harmin.)

particular in the $\pi-\pi$ transitions where both intermediate and final states have $m=0$. Figure 18 shows the results. ${ }^{(118)}$ Experimental works on hydrogen Rydberg states are underway.

\subsection{Perturbative Treatment: Excited Rydberg States}

The perturbative treatment of the Stark effect in atoms has been revived recently, partly because of the advance of higher-order perturbation theory, notably the Bender-Wu theory, ${ }^{(113)}$ and partly because of the use of the Stark effect on excited states to detect highly excited atoms. In particular, a connection between the Bender-Wu theory for high-order Rayleigh-Schrödinger perturbation coefficients for the two-dimensional rotationally symmetric anharmonic oscillator and the behavior of resonances in the hydrogen Stark problem has been made. ${ }^{(114,115)}$ The energy of the Stark effect in hydrogen can be expressed in terms of arbitrarily high orders of Rayleigh-Schrödinger perturbation theory ${ }^{(115)}$

$$
E\left(n_{1}, n_{2}, m, F\right) \sim \sum_{N} E_{n_{1} n_{2} m}^{(N)} F^{N}
$$


The expression in Eq. (58) is known as the Rayleigh-Schrödinger series and $E_{n_{1} n_{2} m}^{(N)}$ is a Rayleigh-Schrödinger coefficient. The coefficients are expressed in terms of parabolic quantum numbers, $n_{1}$ and $n_{2}$, and the magnetic quantum number $m . F$ is the electric field strength. The precise asymptotic behavior of $E_{n_{1} n_{2} m}^{(N)}$ for high-order perturbation theory in $N$ up to 150 has been carried out. ${ }^{(115)}$

\section{Nonhydrogenic Atoms in External Fields}

\subsection{Introduction}

In the following, we discuss magnetic and electric effects of nonhydrogenic atoms in terms of quantum defect theory (QDT). The electron-ion core interaction of nonhydrogenic atoms is represented by the Coulomb potential, $-1 / r$, plus a short-range electrostatic interaction $v(r)$. In the far zone, $r \rightarrow \infty$, the interaction is dominated by the pure Coulomb potential and the wave function is known analytically. In the near zone, $r \leqslant r_{0}$, the electron's interaction with the short-range non-Coulombic potential $v(r)$ is characterized by a parameter. This parameter is fixed by the boundary condition at $r=r_{0}$ for the wave function in the far zone and is identified as the quantum defect or phase shift. This is the underlying principle of quantum defect theory. ${ }^{(21-24)}$

The magnetic and electric potentials are proportional to $r^{n}$, with $n=1$ for electric and $n=2$ for diamagnetic potentials. For nonhydrogenic atoms in external fields, the effective potential has the form $-1 / r+v(r)+a_{n} r^{n}$. The external fields dominate in the far zone whereas $v(r)$ dominates in the near zone. The situation thus appears applicable for a QDT approach. However, the symmetries in these two configuration spaces are different. It is cylindrical symmetry in the far zone where external fields dominate and spherical symmetry in the near zone where the electrostatic field dominates. This difference of symmetry in different configuration spaces requires a transformation between two different coordinate systems. The Stark effect of nonhydrogen atoms provides such an example. The pure Coulomb plus Stark potential is separable in parabolic coordinates and an analytically known wave function exists. Thus the eigenfunctions of a nonhydrogenic atom can be represented by a linear superposition of hydrogenic Stark wave functions in parabolic coordinates. The mixing coefficients are expressed in terms of a reaction matrix and fixed by the boundary conditions in the near zone. The two configuration spaces are related by a local frame transformation. ${ }^{(119)}$ The scattering effect of the short-range electrostatic interaction $v(r)$ of the electron with the ion core is expressed in terms of a quantum defect (or phase shift) which represents the eigenvalue 
of the reaction matrix in the near zone. Fano has succeeded in expressing the photoionization cross section of nonhydrogenic atoms in a Stark field for all energies in terms of quantum defect and frame transformation parameters. ${ }^{(119)}$

However, the situation for the diamagnetic problem is not so simple. We have seen in the previous sections that the Coulomb plus diamagnetic potentials are not separable in any coordinate system. That is, we do not have analytic solutions to represent the far zone region. Thus a unified channel approach for atomic diamagnetism is not yet possible; nonetheless, if the diamagnetic potential can be treated as a perturbation, then the quantum defect treatment can be readily made. In evaluating the matrix elements of external field potentials, it is only important to know the radial functions for large $r$, well outside of the inner core. We should expect to obtain a good approximation for the radial integrals by using the zero-field hydrogenic wave functions with indices $n$ and $l$ and putting $n \equiv \nu$, as discussed in Section 2.2.2b. In fact, Schiff and Snyder ${ }^{(69)}$ have worked out the diamagnetic shifts for the alkalis using this approach. This method leads to the normal diamagnetic scaling law as a quartic power of $\nu$, the effective quantum number, along a single Rydberg series.

For perturbed Rydberg spectra, in particular when the excited electron interacts with an open-shell ion core, a multichannel quantum defect theory (MQDT) is required. ${ }^{(120)}$ We shall begin with a brief discussion of MQDT suitable for the alkaline earths, such as Ba.

For an atom in combined external magnetic and electric fields, the relevant Hamiltonian is

$$
H_{\mathrm{ME}}=\mu_{0}\left(g_{e} \mathbf{L}+g_{s} \mathbf{S}\right) \cdot \mathbf{B}+\frac{e^{2}}{8 m c^{2}}(\mathbf{r} \times \mathbf{B})^{2}-\frac{e}{c}(\mathbf{V} \times \mathbf{B}) \cdot \mathbf{r}-e \mathbf{r} \cdot \mathbf{F}
$$

The first term is the paramagnetic Zeeman potential. It is independent of radial excitation and depends only on spin and orbital angular momentum. The shifts and splittings of spectral lines are the same for all levels of a channel for a given magnetic field $B$. The Landé $g$-factor is also characteristic for all levels belonging to an unperturbed Rydberg channel. These simple systematics are no longer true for perturbed spectra. ${ }^{(24)}$ The last three terms in Eq. (59) are the diamagnetic, motional Stark, and Stark potentials, respectively. Since these three terms depend on the extent of radial excitation, the shifts and splittings vary from level to level. One can control experimental conditions such that only one term in Eq. (59) is important at a time. We shall discuss the electric and magnetic effects separately for perturbed Rydberg atoms in this section. 


\subsection{Quantum Defect Theory of Rydberg Spectra}

Since the most complete diamagnetic spectra are on alkaline earth atoms, ${ }^{(11,38)}$ i.e., $\mathrm{Ba}$ and $\mathrm{Sr}$, we hereby outline a two-channel MQDT model which is suitable for the alkaline earths in order to illustrate the major aspects of the formulas of the quantum defect theory. ${ }^{(120)}$ The formulas can be easily generalized to cases of more than two channels as well as simplified to a single-channel case, such as for the alkalis.

A channel is defined, according to the MQDT, as a set of discrete and continuum states of an ion-electron complex which differ only in the energy of the excited electron. A channel is specified by the orbital, spin, and fine-structure quantum numbers of the ion, the orbital and spin angular momenta of the electron, and the coupling of the electron to the ionic core. For example, all states specified by $\mathrm{Ba} 6 s\left({ }^{2} S_{1 / 2}\right) \varepsilon s, J=0$ for discrete and continuum values of $\varepsilon$ form a singly excited electronic channel, while the set of states specified by $\mathrm{Ba} 5 d\left({ }^{2} D_{3 / 2}\right) \varepsilon d_{3 / 2}, J=0$ comprise a doubly excited electronic channel.

In the case of the $\mathrm{Ba}$ atom, the singly excited Rydberg channels $6 \mathrm{snl}$ converge to the first ionization limit $I_{1}$, corresponding to the $6 \mathrm{~s}$ level of $\mathrm{Ba}^{+}$; whereas the doubly excited channels $5 d n l$ converge to the second ionization limit $I_{2}$, corresponding to the $5 d$ levels of $\mathrm{Ba}^{+}$. At each level position we define two effective quantum numbers, $\nu_{1}$ and $\nu_{2}$, such that

$$
\begin{aligned}
E_{n} & =I_{1}-\mathrm{Ry} / \nu_{1}^{2} \\
& =I_{2}-\mathrm{Ry} / \nu_{2}^{2}
\end{aligned}
$$

where Ry is the Rydberg constant. The quantum defect $\mu$ is defined by $\mu=n-\nu_{1}$. Equation (60) establishes a functional relationship between $\nu_{1}$ and $\nu_{2}$ :

$$
\nu_{1}=\nu_{2}\left[1-\nu_{2}^{2}\left(\frac{I_{2}-I_{1}}{\mathrm{Ry}}\right)\right]^{-1 / 2}
$$

The quantum defect $\mu$ in a multichannel case is not required to be a smoothly varying function of energy. Instead, it can vary from level to level due to interchannel interactions. The zero-field potential between the excited electron and the ion core has the following property:

$$
\begin{aligned}
V(r) & =-\frac{e^{2}}{r}+v(r) \quad \text { for } r<r_{0} \\
& =-\frac{e^{2}}{r} \quad \text { for } r>r_{0}
\end{aligned}
$$


The wave function for the scattering state $e+\mathrm{Ba}^{+}$can be written in the general form

$$
\psi=\sum_{i j} \Phi_{i}\left[f\left(\nu_{i}, r\right) \delta_{i j}-g\left(\nu_{i}, r\right) R_{i j}\right] b_{j} \quad \text { for } r>r_{0}
$$

where $f$ and $g$ are the regular and irregular Coulomb wave functions, respectively, with $\nu_{i}=\nu_{1}$ or $\nu_{2} . R_{i j}$ is the reaction matrix which characterizes the short-range non-Coulombic potential, and the $b_{j}$ are the mixing coefficients which are determined by application of boundary conditions. An energy-dependent $R$-matrix would lead to energy-dependent eigenvalues $\mu_{\alpha}$ and eigenvectors $U_{i \alpha}$, where

$$
R_{i j}(\varepsilon)=\sum_{\alpha} U_{i \alpha}^{\dagger}(\varepsilon) \tan \pi \mu(\varepsilon) U_{\alpha j}(\varepsilon)
$$

For a two-channel case

$$
U_{i \alpha}=\left(\begin{array}{cc}
\cos \theta & \sin \theta \\
-\sin \theta & \cos \theta
\end{array}\right)
$$

In general $U_{i \alpha}$ is an orthogonal matrix which relates the asymptotic channels $i$ applicable when the excited electron is far from the core to the closecoupling channels $\alpha$ applicable when the excited electron penetrates the core. To account for energy-dependent effects we expand the channel mixing angle $\theta$ and the eigen-quantum defect $\mu_{\alpha}$ in energy using the first two terms of a Taylor series expansion

$$
\theta(\varepsilon) \cong \theta_{0}+\varepsilon \theta^{(1)}
$$

with

$$
\theta^{(1)} \equiv \frac{\partial \theta}{\partial \varepsilon}
$$

A similar expression applies for $\mu_{\alpha}$. To obtain discrete energy levels, we impose the boundary condition that $\psi \rightarrow 0$ as $r \rightarrow \infty$, where $\psi$ is the wave function given in Eq. (63). This leads to the consistency relation

$$
F\left(\nu_{1}, \nu_{2}\right)=\operatorname{det}\left|U_{i \alpha} \sin \pi\left(\nu_{i}+\mu_{\alpha}\right)\right|=0
$$

Equations (61) and (65) jointly determine all the discrete levels. Namely, all the discrete levels should lie at the intersections of the curve 
represented by Eq. (61) with that represented by Eq. (65). For each energy level, Eq. (60) determines a pair of values $\left(\nu_{1}, \nu_{2}\right)$ on a two-dimensional plot $\nu_{1}$ vs. $\nu_{2}$. The curve determined by Eq. $(65), F\left(\nu_{1}, \nu_{2}\right)=0$, will pass through and connect all the discrete levels belonging to these two-channels. The parameters $U_{i \alpha}$ and $\mu_{\alpha}$ are introduced through the diagonalization of the reaction matrix $R$ and are determined by matching the wave functions describing the two different configuration spaces, i.e., the wave function for the dissociation channel $i$ appropriate at large distances and that for the close-coupled channel $\alpha$ appropriate at small distances. If these parameters, i.e., $U_{i \alpha}$ and $\mu_{\alpha}$, are energy independent, it is clear that the curve represented by Eq. (65) is periodic on the two-dimensional plot $\nu_{1}$ vs. $\nu_{2}$. Indeed, the analysis of perturbed noble gas Rydberg spectra demonstrates convincingly the periodicity of channel interaction. ${ }^{(24)}$ The interaction of Rydberg channels with doubly excited channels leads to the introduction of energy-dependent parameters and therefore the above periodicity is broken. As we have discussed, energy-dependent parameters are then needed since the doubly excited channels require an additional label to characterize the effect of radial correlations. This effect has been singled out as one of the reasons to study double-excitation using hyperspherical coordinates. Alkaline earth spectra exemplify this situation. Extensive studies have been carried out experimentally ${ }^{(121,122)}$ as well as theoretically $^{(123)}$ for these spectra.

The normalized wave function for $r>r_{0}$ can be represented as a superposition of the dissociation channels $i$ or the close-coupled channels $\alpha$ in the form

$$
\psi_{n}=\sum_{i} \Phi_{i} P_{i}^{(n)}(r) Z_{i}^{(n)}
$$

where $\Phi_{i}$ is the ion-core wave function, $P_{i}^{(n)}(r)$ is the excited electron wave function, and $Z_{i}^{(n)}$ represents the set of expansion coefficients in the $i$-channel representation. $\Phi_{\alpha}, F_{\alpha}^{(n)}(r)$, and $A_{\alpha}^{(n)}$ have similar meanings in the $\alpha$-channel representation. The oscillator strengths of these two mutually interacting channels are represented by ${ }^{(124)}$

$$
\begin{aligned}
f_{n}= & \frac{E_{n}-E_{0}}{\cos ^{2} \theta \sin ^{2} \pi\left(\mu_{1}-\mu_{2}\right)} \\
& \times\left\{D_{1} \sin \pi\left(\nu_{1}+\mu_{2}\right)+D_{2} \sin \pi\left(\nu_{2}+\mu_{1}\right)\right. \\
& \left.\times\left[\frac{d\left(-\nu_{1}\right)}{d \nu_{2}}\right]^{1 / 2}\right\}^{2} /\left\{\nu_{1}^{3}+\nu_{2}^{3}\left[\frac{d\left(-\nu_{1}\right)}{d \nu_{2}}\right]\right\}
\end{aligned}
$$

where $D_{\alpha}, \alpha=1,2$, is the energy independent dipole moment parameter. 
The expression $d\left(-\nu_{1}\right) / d \nu_{2}$ is the slope of the two-dimensional quantum defect plot of $\nu_{1}$ vs. $\nu_{2}$. Its explicit expression in terms of MQDT parameters is

$$
\frac{d\left(-\nu_{1}\right)}{d \nu_{2}}=\frac{1}{\cos ^{2} \pi \nu_{2}} \frac{\Gamma}{\left(\tan \pi \nu_{2}-\alpha\right)^{2}+\Gamma^{2}}
$$

It has a maximum at $\tan \pi \nu_{2}=\alpha$ and a width at half-maximum of $\Gamma$, which measures the interaction strength between these two channels. $\alpha$ and $\Gamma$ depend on the quantum defect parameters $\mu_{\alpha}$ and $U_{i \alpha}$. The expression in Eq. (68) has a symmetrical Lorentz shape, for constant quantum defect parameters, modified by the factor $1 / \cos ^{2} \pi \nu_{2}$. When there is no interaction, $\theta=0$, and $\Gamma \equiv 0$. For the energy level $E_{n}$, having $\nu_{1, n}=n-\mu$, we see clearly from Eqs. (67) and (68) that

$$
f_{n} \rightarrow\left(E_{n}-E_{0}\right)\left|D_{1}\right|^{2} / \nu_{1, n}^{3}
$$

Therefore, for unperturbed Rydberg levels, the oscillator strength scales like $\nu_{1, n}^{-3}$, with $\nu_{1, n}=n-\mu_{1}$. However, when the interaction is strong, the slope $d\left(-\nu_{1}\right) / d \nu_{2}$ can be large and the mixing angle $\theta$ need not be small. In this case the oscillator strength does not follow the $\nu_{1, n}^{-3}$ law.

\subsection{Paramagnetism: Channel Mixing Effects on Magnetic Shifts}

We first consider the situation for magnetic fields which are weak in the sense that the paramagnetic potential is smaller than the Coulomb potential but larger than the diamagnetic interaction. We also assume that the effect due to nuclear spin is negligible. The paramagnetic potential will produce the linear Zeeman shift, $(e \hbar / 2 m) g B M$. The Landé $g$-factor for the $n$th discrete level, $g_{n}$, is given by the expectation value of the operator $g=1+\mathbf{s} \cdot \mathbf{j} / \mathbf{j} \cdot \mathbf{j}$ calculated with the $n$th level's wave function $\psi_{n}$. If we use a MQDT wave function, such as in Eq. (66), the Landé $g$-factor takes the following form ${ }^{(24,125)}$ :

$$
g_{n}=\sum_{i j} g_{i j} Z_{i}^{(n)} Z_{j}^{(n)}=\sum_{\alpha \beta} g_{\alpha \beta} A_{\alpha}^{(n)} A_{\beta}^{(n)}
$$

The $g$ factor may be expressed in terms of two types of channel representation, the $i$ - and $\alpha$-channel representations. The dissociative channel $i$ corresponds to the excited electron at large distance from the ion core, where spin-orbit coupling is likely to be important. Thus it is a good approximation to label the $i$-channel according to a $j j$ coupling scheme. The close-coupled channel $\alpha$, on the other hand, is more suitably character- 
ized by an $L S$ coupling scheme, since the interaction is dominant at distances close to the ion core. Because of channel interactions, the coupling scheme of a perturbed Rydberg series will depend on the degree of mixing among the relevant channels. This information is imbedded in the mixing coefficients $A_{\alpha}$ or $Z_{i}$. The Landé $g$-factor depends only on the angular momentum coupling scheme and it is diagonal in an $L S$ coupling scheme. Values of $g$-factors, measured by linear Zeeman effect experiments, have been utilized as a sensitive probe of the angular momentum coupling scheme of a given spectral line. One therefore expects that the measured $g$-values along a perturbed Rydberg series would reflect the degree of channel mixing.

A recent set of measurements of the $g$-factor along a series of $J=2$ Rydberg states having the configurations $5 s n d$ in $\mathrm{Sr}$ in the region of strong mixing between the ${ }^{1} D_{2}$ and ${ }^{3} D_{2}$ Rydberg series, shows a variation which is in agreement with the predictions of MQDT. ${ }^{(125)}$ Figure 19 shows the results. Esherick ${ }^{(125)}$ uses a five-channel MQDT for the channels, 5snd ${ }^{1} D_{2}, 5 s n d{ }^{3} D_{2}, 4 d 5 s{ }^{1} D_{2}, 4 d 5 s{ }^{3} D_{2}$, and $4 p^{2}{ }^{1} D_{2}$ to fit his data. The quantum defect parameters, $U_{i \alpha}$ and $\mu_{\alpha}$, thus obtained are used to compute the mixing coefficients $\boldsymbol{A}_{\alpha}^{(n)}$. A pure $L S$ coupled scheme is used to evaluate $g_{\alpha}$. Equation (69) is used to compute the $g_{n}$ along the series. From Figure 19 , one notes that the strongest mixing occurs for $n=16$, and the $g$-factors vary between 1 (that for a pure ${ }^{1} D_{2}$ state) and $7 / 6$ (that for a pure ${ }^{3} D_{2}$ state). This success in treating the variation of $g$-factors over the whole perturbed series as a single problem rather than dealing with one state at a time, demonstrates the power of MQDT.

Figure 19. $g$-factor as a function of $\nu\left({ }^{2} D_{3 / 2}\right)$, the effective principal quantum number measured relative to the $4 d^{2} D_{3 / 2}$ ionization threshold at $60488.09 \mathrm{~cm}^{-1}$. The solid line is the theoretical prediction and the points correspond to experimental measurements for the bound states designated by 5snd. (From Ref. 125.)

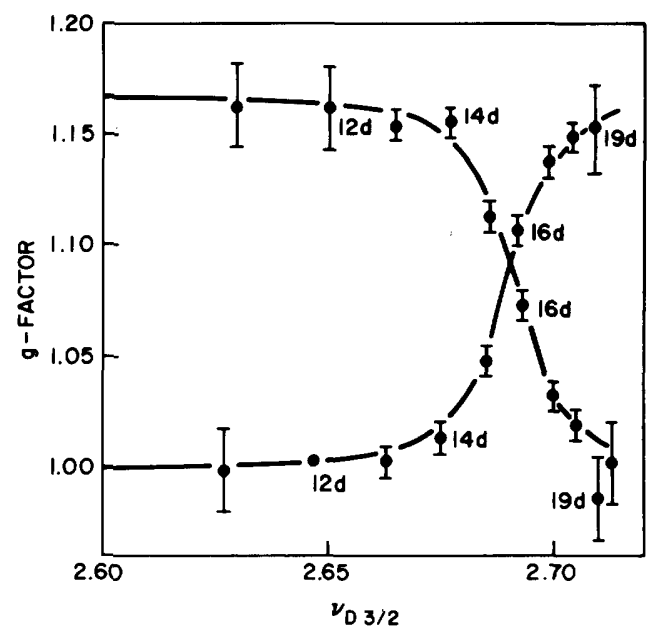




\subsection{Diamagnetism: Magnetic Contribution to Channel Mixing}

In this section, we will discuss in detail how to disentangle complex perturbed Rydberg spectra by measuring the diamagnetic shifts. ${ }^{(120)} \mathrm{We}$ choose the alkaline earth atoms as examples. The alkaline earth spectra are typified by singly excited Rydberg series which overlap series of doubleexcited states. This circumstance makes the identification of the spectral lines difficult. For a given excitation energy, a singly excited Rydberg state has a larger radial extension from the ion core than a doubly excited state. However, channel interaction between these two types of states diminishes the distinction. In fact, the mixings in alkaline earth atoms are so strong as to "hybridize" the spectra in the sense that the oscillator strengths are redistributed between these two types of channels throughout the whole channel including both discrete and continuum portions. The MQDT has been rather successful in analyzing these spectra by introducing energydependent parameters for both $\mu_{\alpha}$ and $\theta .^{(121,126)}$

The $\mathrm{Ba}$ even-parity spectrum is ideal for our study, since the doubly excited channels having configurations $5 d n d$ and $5 d n s$ are embedded among the singly excited Rydberg channels having configurations 6 snd and $6 s n s$. The spectrum has been analyzed by MQDT, ${ }^{(122)}$ and the diamagnetic shifts have been measured. ${ }^{(8)}$ The upper part of Figure 20 shows the two-dimensional quantum defect plot of $\nu_{1}$ vs. $\nu_{2}$ for the even-parity Ba spectrum. The pair of parabolic curves representing the channel interaction of the Rydberg channel $6 s n s{ }^{1} S_{0}$ with the doubly excited channel $5 d n d{ }^{1} S_{0}$ are indicated in the figure. Note that the quantum defects of the Rydberg levels belonging to the 6 sns series are nearly constant (having $\mu_{1} \sim 0.2$ ) except near the doubly excited level $5 d 7 d{ }^{1} S_{0}$, which is marked by a cross in the figure. The Rydberg level $18 s$ (with $\mu_{1} \sim 0.1$ ) is nearly degenerate with $5 d 7 d$ (with $\mu_{1} \sim 0.4$ ) and both are mixed strongly with each other as indicated by the big change in quantum defect relative to those of their neighbors. The channel interactions within $J=2$ are more complex. The solid curves representing the situation for five interacting channels, 6 snd ${ }^{1,3} D_{2}, 5 d n d{ }^{1} D_{2},{ }^{3} F_{2}$, and $5 d n s{ }^{1} D_{2}$ are also indicated in the same figure. The mixing coefficients $Z_{i}^{(n)}$ and the quantum defect parameters, $\mu_{\alpha}$ and $U_{i \alpha}$ for $J=0$ and $J=2$ channels have been obtained by a MQDT fitting to the data. ${ }^{(122)}$ They will be used to evaluate the diamagnetic shifts. The spacing between neighboring Rydberg levels for $n=30$ is $\Delta E_{30,29} \cong$ $10 \mathrm{~cm}^{-1}$, whereas the diamagnetic shift for the $n=30$ level at a magnetic field strength of $B=40 \mathrm{kG}$ is $\Delta \sigma \cong 5 \mathrm{~cm}^{-1}$. We thus have $\Delta E_{30,29}>\Delta \sigma$, and hence we can use perturbation theory to evaluate the diamagnetic shifts.

We use the MQDT wave functions as the zero-field unperturbed wave functions. The diamagnetic potential $H_{D}=\left(e^{2} / 8 m_{e} c^{2}\right) B^{2} r^{2} \sin \theta$ mixes 


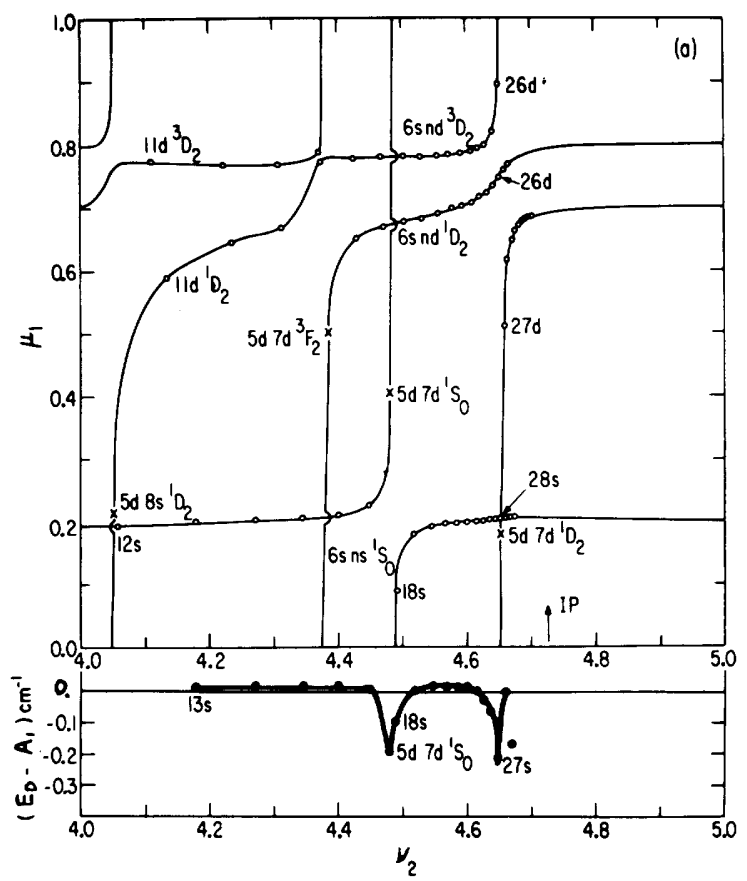

Figure 20. Diamagnetic shifts of the perturbed spectrum of $\mathrm{Ba}$ atoms. The upper figure shows the twodimensional quantum defect plot $\mu_{1}$ vs. $\nu_{2}$ of even parity, $J=0$ and $J=2$ channels. The relevant channels and levels are marked in the figure. The lower figure shows the difference of the diamagnetic shift from the hydrogenic value, $E_{D}-A_{1}$. The solid dots are experimental data from Ref. 8 and the solid curve results from calculations using Eqs. (70) and (76). (From Ref. 120.)

levels with orbital angular mementum $l$ such that $\Delta l=\left|l^{\prime}-l\right|=0,2, \ldots$, and having the same magnetic quantum number $m$ and parity. Because of its $r^{2}$ dependence, the diamagnetic potential is negligible near the ion core. This situation is well suited for the MQDT wave functions in Eq. (66), because in evaluating the matrix elements, it is only important to know the radial function for large $r$, outside of the inner ion core. We expect to obtain a good approximation for the radial integrals by using the Coulomb wave functions with dissociation channel indices $i, n$, and $l$, and putting $n=\nu$ after the integrals are evaluated. For $\mathrm{Ba}$ even-parity states, we choose channel 1 as $6 s n s^{1} S_{0}$ and channel 2 as $5 d n d{ }^{1} S_{0}$. The first-order diamagnetic 
energy for a two-channel perturbed Rydberg spectrum is

$$
\begin{aligned}
\frac{e^{2}}{8 m_{e} c^{2}} B^{2}\left\langle r^{2} \sin ^{2} \theta\right\rangle_{n}= & A_{1}\left[1+\frac{A_{2}}{A_{1}}\left(\frac{\nu_{2}}{\nu_{1}}\right)^{3} \frac{d\left(-\nu_{1}\right)}{d \nu_{2}}\right] \\
& \times\left[1+\left(\frac{\nu_{2}}{\nu_{1}}\right)^{3} \frac{d\left(-\nu_{1}\right)}{d \nu_{2}}\right]^{-1}
\end{aligned}
$$

where $A_{1}$ and $A_{2}$ represent hydrogenic diamagnetic matrix elements for channels 1 and 2, respectively. The cross term between $6 s n s$ and $5 d n d$ vanishes since the core states $6 s$ and $5 d$ are orthogonal, i.e., $\langle 6 s \mid 5 d\rangle \equiv 0$. The hydrogenic diamagnetic matrix element for an $n, l, m=0$ state is ${ }^{(69)}$

$$
A_{l, l}(n, 0)=+4.97 \times 10^{-15} B^{2} \frac{n^{2}\left[5 n^{2}+1-3 l(l+1)\right]\left(l^{2}+l-1\right)}{(2 l+3)(2 l-1)}
$$

and the off-diagonal matrix element is

$$
B_{l, l-2}(n, 0)=-4.97 \times 10^{-15} B^{2} \frac{5 n^{2} l(l-1)}{2(2 l-1)}\left\{\frac{\left(n^{2}-l^{2}\right)\left[n^{2}-(l-1)^{2}\right]}{(2 l+1)(2 l-3)}\right\}^{1 / 2}
$$

The explicit forms of $\boldsymbol{A}_{1}$ and $\boldsymbol{A}_{2}$ can be obtained easily by replacing $n \rightarrow \nu_{1}, l=0$ for channel 1 and $n \rightarrow \nu_{2}, l=2$ for channel 2 in Eq. (71), respectively:

$$
\begin{aligned}
& A_{1}=A_{s}\left(\nu_{1}\right)=4.97 \times 10^{-15} B^{2} \frac{\nu_{1}^{2}\left(5 \nu_{1}^{2}+1\right)}{3} \\
& A_{2}=A_{d}\left(\nu_{2}\right)=4.97 \times 10^{-15} B^{2} \frac{5 \nu_{2}^{2}\left(5 \nu_{2}^{2}-17\right)}{21}
\end{aligned}
$$

The energy matrix elements are expressed in units of $\mathrm{cm}^{-1}$ and $B$ is in gauss.

For negligible channel interaction, the slope of the $\nu_{1}$ vs. $\nu_{2}$ plot in Figure 20 is $d\left(-\nu_{1}\right) / d \nu_{2} \approx 0$ at an eigenvalue belonging to channel 1 , and $d\left(-\nu_{1}\right) / d \nu_{2} \gg 1$ for $\varepsilon=\nu_{2 n}$ at an eigenvalue belonging to channel 2. Thus, the shift expressed by Eq. (70) becomes $E_{D}^{(1)} \approx A_{1} \approx \nu_{1}^{4} B^{2}$ at an eigenvalue belonging to channel 1 and $E_{D}^{(1)} \approx A_{1} \approx \nu_{2}^{4} B^{2}$ at an eigenvalue belonging to channel 2 . Therefore the larger the radial excitation the bigger the diamagnetic shift. Since the diamagnetic potential is always positive, the shift is to the blue. For the Ba atom the doubly excited level $5 d 7 d^{1} S_{0}$ lies between the levels $6 s 17 s$ and $6 s 18 s$ so that all three are nearly degenerate 
in energy. Without interaction, the doubly excited level $5 d 7 d$ is much more compact in radial size than the neighboring Rydberg levels, therefore it is expected that the doubly excited level $5 d 7 d$ should have a smaller diamagnetic blue shift than the $17 \mathrm{~s}$ and $18 \mathrm{~s}$ levels. Also the $18 \mathrm{~s}$ should have a larger blue shift than that of $17 \mathrm{~s}$. Channel interaction changes the above simple picture and the shift is described by Eq. (70). The quantum defect parameters needed to evaluate the slope $d\left(-\nu_{1}\right) / d \nu_{2}$ have been obtained by fitting to the energy levels. ${ }^{(120)}$ For a field of $B=35.7 \mathrm{kG}$, calculations based on Eq. (70) give diamagnetic shifts for $17 s, 5 d 7 d$, and $18 s$ of 0.29 , 0.17 , and $0.28 \mathrm{~cm}^{-1}$, respectively. It is interesting to note that the $18 \mathrm{~s}$ level actually has a shift which is smaller than that of the $17 \mathrm{~s}$ level because of the channel mixing with the more compact doubly excited $5 d 7 d$ level. The lower portion of Figure 20 shows the difference between the diamagnetic shifts for $\mathrm{Ba}$ and those for the corresponding levels of hydrogenlike atoms, $E_{D}-A_{1}$, along the $6 s n s{ }^{1} S_{0}$ series. Note that the $n s$ levels $(n=13$ to 17$)$ are relatively unperturbed Rydberg levels whereas $5 d 7 d$ and $18 s$ are perturbed.

When the Rydberg excitation reaches $\nu_{n} \approx 30$, diamagnetic $l$-mixing becomes important for field strengths $B \approx 40 \mathrm{kG}$. A second-order perturbation calculation of the energy shifts is required ${ }^{(120)}$ :

$$
E_{\nu_{n} l}^{(2)}=\sum_{\nu_{n}^{\prime} l^{\prime}} \frac{\mid\left\langle\left.\nu_{n} l\left|H_{D}\right| \nu_{n}^{\prime} l^{\prime}\right|^{2}\right.}{E_{\nu_{n} l}-E_{\nu_{n}^{\prime} l^{\prime}}}
$$

We consider only states with $\nu_{n}=\nu_{n}^{\prime}$ and $l^{\prime}=l+2$. We have

$$
E_{\nu_{n} l}-E_{\nu_{n}^{\prime} l^{\prime}} \cong \frac{\operatorname{Ry}\left(\mu_{l}^{\prime}-\mu_{l}\right)}{\nu_{n}^{3}}
$$

where $\nu_{n}=n-\mu_{l}$ and $\nu_{n}^{\prime}=n-\mu_{l}^{\prime}$. Again we use MQDT wave functions to evaluate the matrix element in Eq. (73):

$$
\left\langle\nu_{n} l\left|H_{D}\right| \nu_{n}^{\prime} l^{\prime}\right\rangle=\sum_{i} Z_{i}\left(\nu_{i}^{\prime}, l\right) Z_{i}\left(\nu_{i}^{\prime}, l^{\prime}\right) B_{l, l}^{\prime}\left(\nu_{i}, 0\right) \delta_{l, l-2}
$$

Here $B_{l, l^{\prime}}\left(\nu_{i}, 0\right)$ is the off-diagonal matrix element of Eq. (72) in which we have made the replacement $n \rightarrow \nu_{i}$ and $\nu_{i} \cong \nu_{i}^{\prime} ; \nu_{1}$ and $\nu_{2}$ are the effective quantum numbers defined in Eq. (60) for different ionization limits; and $Z_{i}$ is the mixing coefficient, defined in Eq. (66), which describes the extent of channel interactions. In order to see the diamagnetic $l$-mixing, we consider matrix elements connecting $\mathrm{Ba} 6 s n s$ levels with $\mathrm{Ba} 6$ snd levels, all having $m=0$. We make use of Eqs. (73), (74), and (75) to obtain the 
second-order diamagnetic energy shift:

$$
\begin{aligned}
E_{D}^{(2)}= & \frac{\left(4.97 \times 10^{-15}\right)^{2}}{\operatorname{Ry}} \frac{5}{9} \cdot \frac{B^{4} \nu_{1 l}^{11}}{\mu_{l}^{\prime}-\mu_{l}} \cdot\left[1+\left(\frac{\nu_{2}}{\nu_{1}}\right)^{3} \frac{d\left(-\nu_{1}\right)}{d \nu_{2}}\right]_{l}^{-1} \\
& \times\left[1+\left(\frac{\nu_{2}}{\nu_{1}}\right)^{3} \frac{d\left(-\nu_{1}\right)}{d \nu_{2}}\right]_{l^{\prime}}^{-1}
\end{aligned}
$$

Here we have put $\nu_{l}=\nu_{l}^{\prime}, l=0$ and $l^{\prime}=2$. The zero-field channel interactions have been taken into account through the slopes, $\left[d\left(-\nu_{1}\right) / d \nu_{2}\right]_{\text {l, }}$, for $6 s n s, J=0$ and $6 s n d, J=2$ channels.

For noninteracting Rydberg spectra, $\left[d\left(-\nu_{1}\right) / d \nu_{2}\right]=0$ for $l=0, J=0$ and $l=2, J=2$. The second-order shift is proportional to $B_{\nu_{1}}^{4}{ }^{(11)}$ and inversely proportional to the difference in quantum defects, $\Delta=\mu_{l}-\mu_{l}$, of neighboring $l$-mixed levels. For field strengths $B=35.7 \mathrm{kG}$, the $l$-mixing will not be important until $\nu_{1} \approx 30$. A MQDT calculation of $l$-mixing based on Eqs. (70) and (76) has been carried out along 6 sns series and the result is shown in Figure 20. The anomalous diamagnetic shifts around $27 \mathrm{~s}$ are due to the $l$-mixing between the $6 s n s$ channel and the $6 s n d$ channels. The $6 s n d$ channels are perturbed by the interloping level $5 d 7 d^{1} D_{2}$, as demonstrated by the zero-field quantum defect plot in Figure 20. Once again the compactness of the $5 d 7 d^{1} D_{2}$ state is reflected in the smaller diamagnetic shift relative to its neighboring levels. Therefore, the diamagnetic interaction can be used as a probe to measure the extent of radial excitation and to disentangle complex perturbed Rydberg spectra.

\section{The Quasi-Landau Resonances and the Role of the Quantum Defect}

We turn now to the discussion of the role of the quantum defect on the shapes of quasi-Landau resonances. ${ }^{(11)}$ The energy spacings between quasi-Landau resonances are determined by the effective potential, comprising the Coulomb and diamagnetic potentials, over a very large range of the coordinate perpendicular to the magnetic field direction $z$, as shown by the WKB results. ${ }^{(16,35,36)}$ Thus the spacings do not depend on the detailed short-range interaction between the excited electron and the ion core. Essentially, the $3 / 2 \hbar \omega$ spacing calculated in WKB approximation is universal for all atoms, in agreement with all the experimental findings, as discussed in Section 2. However, the electron-core short-range interaction represented by the quantum defect, will affect the shapes of these resonances. This is seen in Figure 1, where the shapes of the resonances for $\mathrm{Sr}$ are markedly different from those for $\mathrm{Ba}$. In the quasi-Landau region, the electron is moving along the potential ridge formed by the Coulomb and 
diamagnetic potentials. The motion of the electron is stationary when it is in the direction $\rho$ perpendicular to the magnetic field direction. The spherically symmetric Coulomb potential couples the motion in $\rho$ with that in $z$ and eventually directs the electron's motion along the $z$ axis and escapes, with a characteristic time $\tau$. Since the Coulomb potential is strongest near the origin, this coupling strength will be sensitive near the origin as well. Since the short-range electron-ion core interactions vary from atom to atom so will the shapes of the quasi-Landau resonances. These resonances can be characterized, in addition to their energies, by two parameters, the phase shift $\phi$ and the width $\Gamma$. The phase shift $\phi$, due to electron-core interaction, is defined relative to the hydrogenic value, which has a phase shift $\phi_{0}$. The width $\Gamma$ measures the lifetime, $\tau=\hbar / \Gamma$, of these resonances. $\phi$ and $\Gamma$ are related near the resonance by $\tan \left(\phi-\phi_{0}\right)=\Gamma / 2\left(E-E_{0}\right)$, where $E_{0}$ is the resonance position. With the above physical picture, we can now interpret the observations shown in Figure 1.

For the Sr principal series, the zero-field spectrum near the threshold is dominated by $5 \operatorname{sep}{ }^{1} P_{1}^{0}$ and perturbed by a strongly bound $4 d 5 p^{1} P_{1}^{0}$ state. ${ }^{(124)}$ The effect of $4 d 5 p{ }^{1} P_{1}^{0}$ on the quasi-Landau resonances is to produce a small phase shift $\phi$, and thus produce a finite width $\Gamma$. On the other hand, the zero-field spectrum near the ionization threshold in $\mathrm{Ba}$ is dominated by $5 d 8 p^{1} P_{1}^{0}$. The interaction of $5 d 8 p^{1} P_{1}^{0}$ with the continuum background $6 s{ }^{1} P_{1}^{0}$ produces a large phase shift $\phi$ and thus a greater width $\Gamma$.

\subsection{The Stark Effect: Coupling of Parabolic Channels by Scattering from the Ion Core}

Recently, Fano ${ }^{(119)}$ has succeeded in formulating a nonperturbative theory of the Stark effect of nonhydrogenic Rydberg atoms in terms of the quantum defect and local frame transformation parameters. The extension of the theory of the Stark effect to atoms other than hydrogen requires the addition of a short-range non-Coulombic potential $v(r)$ representing the effect of the ionic core on the excited electron.

The eigenfunctions $\left|n^{\prime} m\right\rangle$ for the potential $-e^{2} / r+e \mathbf{F} \cdot \mathbf{r}+v$ are obtained by a linear superposition of the eigenfunctions in Eq. (53) with different $n$, but same $m$. The mixing coefficients are presented by a reaction matrix $K$ in parabolic coordinates. ${ }^{(119)}$ The short-range electron-core interaction is dominated by the non-Coulombic potential which produces a phase shift $\delta_{l}$ or a quantum defect $\mu=\delta_{l} / \pi$ in the electron's wave function. The phase shift $\delta_{l}$ is the eigenvalue of the reaction matrix $K$ represented in the space of orbital angular momentum $l$

$$
N_{l \varepsilon}^{*} K_{l l}(\varepsilon) N_{l \varepsilon}=-\tan \delta_{l}(\varepsilon)
$$


Fano ${ }^{(119)}$ has succeeded in transforming the $K$-matrix from the spherically symmetric frame having orbital quantum number $l$ into the cylindrically symmetric frame of parabolic coordinates by a local frame transformation

$$
K_{n n^{\prime}}(\varepsilon)=\sum_{l} \alpha_{l m}^{*}\left(\beta_{n}, \nu\right) K_{l l}(\varepsilon) \alpha_{l m}\left(\beta_{n^{\prime}}, \nu\right)
$$

where $n$ represents the parabolic quantum number. The local frame transformation is performed in the region where the field strength $F$ is not important, namely, as $(\xi, \eta) \rightarrow 0$ and $r \rightarrow 0$.

The photoionization cross section is proportional to

$$
\sum_{n^{\prime} n} D_{n^{\prime} m}^{*}(\varepsilon)\left\{1+\left[N_{\varepsilon}^{*} K(\varepsilon) N_{\varepsilon}\right]^{2}\right\}_{n^{\prime} n}^{-1} D_{n m}(\varepsilon)
$$

where $D_{n m}(\varepsilon)=N_{n \varepsilon}^{*} \alpha_{l m}^{*}\left(\beta_{n}, \nu\right) d_{l m}(\varepsilon)$. The photoionization cross section for atoms other than hydrogen is expressed in terms of the quantum defect in Eq. (77) and the frame transformation parameters of (78). Theoretical calculation has been carried out by Harmin, ${ }^{(118,119)}$ and compared favorably with experiments. ${ }^{(107)}$

For the hydrogen atom, the quantum defect is zero, i.e., $\delta_{l} \equiv 0$ and Eq. (79) reduces to

$$
\sum_{n^{\prime} n} D_{n^{\prime} m}^{*}(\varepsilon) \delta_{n^{\prime} n} D_{n m}(\varepsilon)=\sum_{n} D_{n m}^{2}
$$

and it reproduces the photoionization cross section for hydrogen given by Eq. (56).

\section{Competition of Magnetic and Electric Forces}

\subsection{Introduction}

In the last section, we have discussed various magnetoelectric effects, paramagnetic, diamagnetic, Stark, and motional Stark, on excited atoms separately. These potentials (other than paramagnetic) have one thing in common, namely, they are all long-range potentials, tending asymptotically as $\sim r^{n}$, where $n=1$ and 2 for electric and magnetic fields, respectively. For a given atom and a fixed field strength, the effect scales according to principal quantum number $n$ as $n^{\alpha}$, where $\alpha \geqslant 2$, but with different magnitude. The effects are enriched by the different symmetry and/or constants of motion imposed by the external fields on the otherwise isotropic atoms.

We shall discuss here conditions under which the spectroscopic observables, i.e., level shifts, splittings, and intensities, undergo changes along a 
Rydberg series in terms of external field strength, symmetries, and atomic species. These changes occur, for example, in the case of the diamagnetic effect $^{(5,11)}$ for a field strength $B \approx 5 \times 10^{4} \mathrm{G}$, from the $l$-mixing region for principal quantum number $n \leqslant 30$ through the $n$ and $l$-mixing region and up to the quasi-Landau region with $\frac{3}{2} \hbar \omega_{c}$ spacing around the zero-field ionization limit (see Figure 1). In the $l$-mixing region, the diamagnetic potential acts as a perturbation on the Coulomb dominated potential between the excited electron and the ion core and breaks the isotropy of the unperturbed atom by intermixing the $l$-components. It preserves, however, the parity and cylindrical symmetry of the state.

For the same field strength, as $n$ increases, the relative magnitude of the Coulomb versus diamagnetic potential also changes. The Rydberg electron's Coulomb binding energy is reduced whereas the diamagnetic energy is enhanced. The magnetically induced $l$-mixirg manifolds belonging to different principal quantum numbers $n$ would interact for $n \geqslant 30$. This is the $n$ and $l$-mixing region. ${ }^{(5,11)}$ The $n$ and $l$-mixing becomes complete for electron excitation near the zero field ionization limit where the joint action of Coulomb and diamagnetic potentials forms a "potential ridge". ${ }^{(44,45)}$ The quasi-Landau resonances correspond to the electron's motion propagating along the potential ridge and its orbit being confined in the direction perpendicular to the magnetic field. As a result of the competition between Coulomb and diamagnetic potentials, the spectral lines regroup themselves from the $n$ and $l$-mixing region into the $\frac{3}{2} \hbar \omega_{c}$ spacing of the quasi-Landau region. It is now clear that there is a geometric symmetry as far as the electron's motion in relation to the potential ridge is concerned. However, what mechanism controls the transition of the electron's motion from the $n$ and $l$-mixing region into the quasi-Landau region is not yet clear. Such competition of potentials in changing the excited electron's motion seems to be a general phenomena. We shall discuss specific examples involving both magnetic and electric fields. We shall discuss first the role of motional Stark effect on diamagnetism and then the effects due to crossed external electric and magnetic fields.

\subsection{The Induced Stark Effect: Coupling of Magnetic Sublevels by Nuclear Motion}

It has been known for some time that for an excited atomic system in an external uniform magnetic field, there is an induced electric field due to the motion of the whole atom, i.e., the motional Stark effect. The strength of this term is rather weak compared with the magnetic effect and it is customary to neglect it. However, the conspicuous effects of this term were not appreciated until quite recently. ${ }^{(13,127)}$ Briefly, for a given atom, say 
$\mathrm{Li}$, with mass $M$, temperature $T \approx 10^{3 \circ} \mathrm{K}$, and magnetic field strength $B \approx 5 \times 10^{4} \mathrm{G}$, the induced motional Stark field is $E \approx 70 \mathrm{~V} / \mathrm{cm}$. The effect can be perturbative or violent depending on the spectral range. Figure 21 shows the Li ground-state photoabsorption spectrum in this magnetic field. For $n \leqslant 20$, the effect is basically perturbative, namely, the motional Stark effect induces only the weak parity violating $l$-mixing spectra, i.e., even $l$ components, $l=0$ and 2 , and the $m$-mixing components, i.e., $m=0,+2$, in addition to the $l=1$ and $m=+1$ spectra for right-hand circularly polarized light for normal allowed diamagnetic spectra. These weak forbidden components are documented in Figure 22 by a straightforward diagonalization calculation. ${ }^{(13)}$ In the second region, $30 \leqslant n \leqslant 20$, the

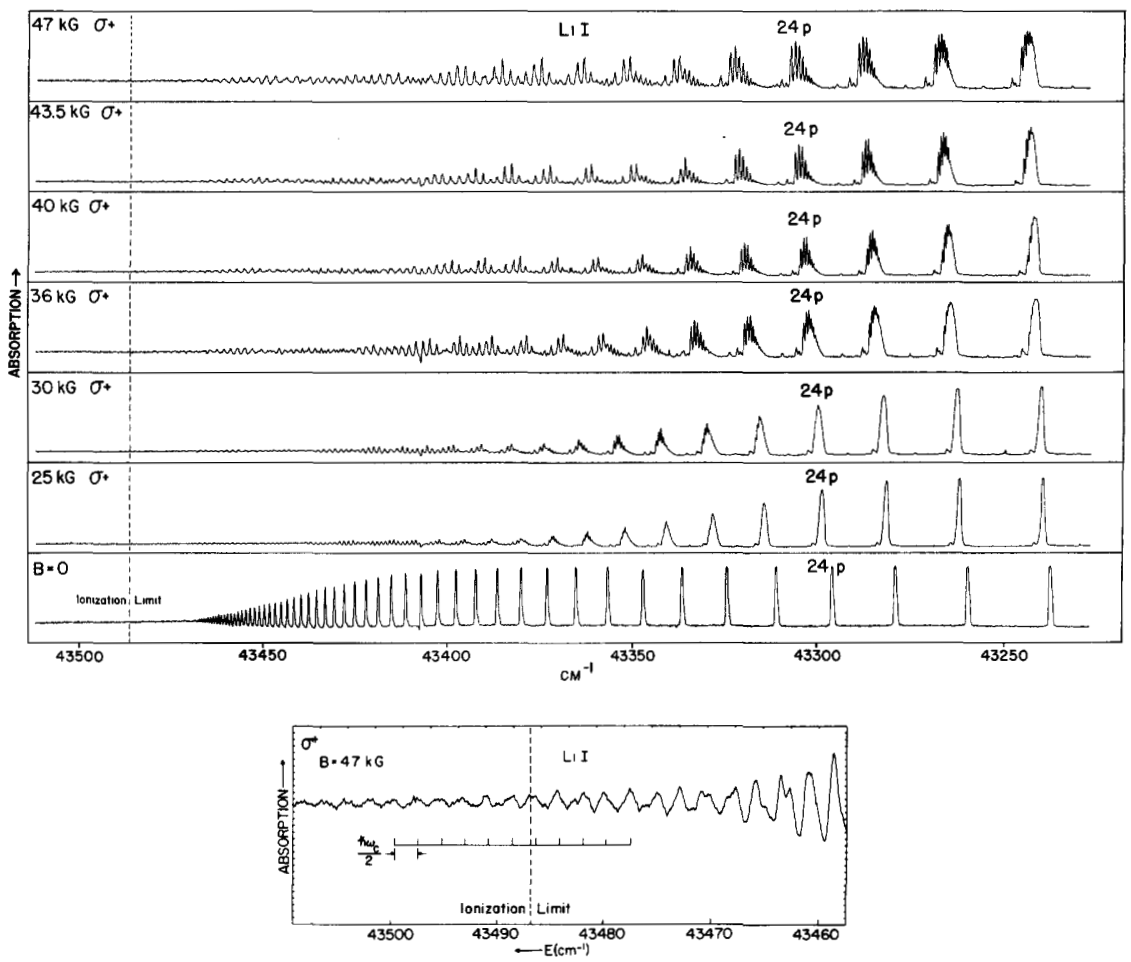

Figure 21. (a) Microdensitometer recordings of the plate transmission of the Li principal series with magnetic field strengths $B=47,43.5,40,36,30$, and $25 \mathrm{kG}\left(\sigma^{+}\right.$polarization) and $B=0$. The $\mathrm{Li}$ vapor pressure is $\approx 0.1$ Torr. (b) Enlarged microdensitometer tracing of $\mathrm{Li}$ in the region across the ionization limit with $B=47 \mathrm{kG}$ and $\sigma^{+}$polarization. The $\mathrm{Li}$ vapor pressure is $\approx 0.7$ Torr. (From Ref. 13.) 
strength of the forbidden components induced by the motional Stark effect begins to be comparable to that of those due to the diamagnetic effect. In the third region, $n \geqslant 30$, the spectra are dominated by the motional Stark effect and altered by the quasi-Landau spacings from $\frac{3}{2} \hbar \omega_{c}$ into $\frac{1}{2} \hbar \omega_{c}$ (cf. Figure 21b).

A perturbative estimation ${ }^{(15)}$ of the amplitude ratio of optical transitions to states with $m \neq 1$ and $m=1$ serves as a criterion to illustrate the results discussed above. This ratio is given by the ratio of the matrix element of the perturbation energy, $m_{e} V_{\perp} W_{c \chi}$, to the energy difference of successive Zeeman levels with $|\Delta m|=1$, i.e., $\mu_{0} B=\frac{1}{2} \hbar \omega_{c}$ :

$$
P=\left[\frac{m_{e} V_{\perp} W_{c}\left\langle n, l,\left.m\right|_{\chi} \mid n, l+1, m \pm 1\right\rangle\left(1+D_{n}\right)}{\frac{1}{2} \hbar \omega_{c}+R\left(2 / n^{3}\right) \Delta \mu}\right]^{2} \sim \frac{m_{e}}{M} \cdot \frac{k T}{R} n^{4}
$$

Here $V_{\perp}=\left|\mathbf{V}_{\mathrm{th}} \times \hat{B}\right|, \frac{1}{2} M V_{\perp}^{2} \approx k T$ and $R=\hbar^{2} / 2 m_{e} \alpha_{0}^{2}=13.6 \mathrm{eV}$. The effect of the quantum defect is taken care of in $D_{n}$ and $\Delta \mu$ as discussed in Section 4 . For $\mathrm{Li}$, this ratio is about 0.1 for $n \approx 20$ but increases to 1.0 for $n>30$. At this point the $m$-mixing due to the motional Stark effect is complete. The diamagnetic interaction creates a series of quasi-Landau levels with different quantum number $n_{L}$ having a spacing $\left(\frac{3}{2}\right) \hbar \omega_{c}$ for a

Figure 22. Li absorption spectrum for the $n=21$ manifold in a magnetic field $B=47.8 \mathrm{kG}$. The curves are densitometer traces from photoabsorption measurements taken at a vapor pressure of $0.1 \mathrm{~mm}$. The calculated values of positions corresponding to different nominal $m$ components, $m=1,2$, or 3 are marked. The vertical lines represent calculated values of the square of the eigenvector component belonging to $l=1, \quad m=1$ allowed transitions. Experimental line centers, the $B=0$ position $\left(\sigma_{0}=43237.31 \mathrm{~cm}^{-1}\right)$ of $n=21$, and the magnitude of the linear Zeeman shift $\left(\mu_{0} B\right)$ for $B=$ $47.8 \mathrm{kG}$ are indicated. The absorption features with $m=3$ and 2 become far more prominent in spectra at higher pressures. (From Ref. 13.)

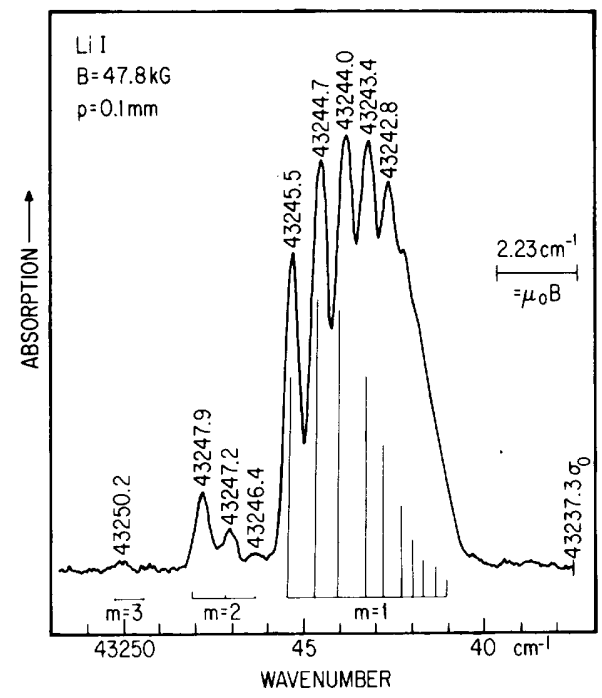




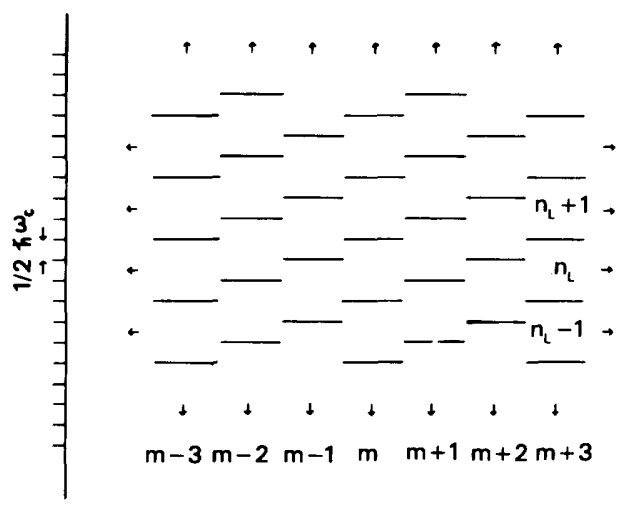

Figure 23. Array of levels $E_{n_{L} m}=$ $\left(m+3 n_{l}\right) \mu_{0} B$. (From Ref. 15.)

specific value of $m$. The motional Stark effect generates different $m$ components due to strong $m$-mixing. New series of quasi-Landau levels belonging to different $m$ components are formed and are displaced by the linear Zeeman shift of orbital levels by $\mu_{0} B=\frac{1}{2} \hbar \omega_{c}$. This picture produces a two-dimensional array of levels, $E_{n_{L} m}=\left(m+3 n_{L}\right) \mu_{0} B$, as shown in Figure 23. This interpretation explains the $\frac{1}{2} \hbar \omega_{c}$ spacing in the photoabsorption spectra of $\mathrm{Li}$ vapor in high magnetic fields. ${ }^{(15)}$

The two-dimensional array of levels can be described by an eigenvalue problem in terms of a two-dimensional finite difference equation:

$$
\left(H_{n_{L} m}-E_{n_{L} m}\right) U_{m}^{n_{L}}=\sum_{n_{L^{\prime}}}\left[A_{n_{L^{\prime}} n^{m}}^{m, m+1} U_{m+1}^{n_{i}^{\prime}}+A_{n_{L} n_{L}}^{m, m-1} U_{m-1}^{n_{L}^{\prime}}\right]
$$

This expression preserves the translational invariance in both $n_{L}$ and $m$ variables where $A_{n_{L} n_{L}^{\prime}}^{m m^{\prime}}=A_{n_{L} n_{L}^{\prime}}^{m m^{\prime}} \delta_{m^{\prime}, m+1}$ represents a two-dimensional tridiagonal matrix. If the values of $n_{L}$ and $m$ extend to infinity, the coupling $A_{n_{L} n_{L}}^{m m^{\prime}}$ will be uniform. One can show by induction that the eigenvalues of Eq. (82) are equally spaced, $E_{n_{L} m}=\left(m+3 n_{L}\right) \mu_{0} B$, and the eigenvectors are uniform. This indicates that the motional Stark resonances are not only equally spaced with spacing $\frac{1}{2} \hbar \omega_{c}$, but each resonance is of equal intensity.

\subsection{Crossed External Electric and Magnetic Fields: Transitions between $\frac{3}{2} \hbar \omega_{c}$ and $\frac{1}{2} \hbar \omega_{c}$ Spacing}

It is only natural now to study the competition of forces of an excited electron under the combined influence of Coulomb, magnetic, and electric fields. ${ }^{(120)}$ The effective potential is

$$
V=-\frac{e^{2}}{r}+a\left(x^{2}+y^{2}\right)-b x=-\frac{e^{2}}{r}+a\left[\left(x-\frac{1}{2} \frac{b}{a}\right)^{2}+y^{2}\right]-\frac{1}{4} \frac{b^{2}}{a}
$$


where $a=\frac{1}{8} m_{e} \omega_{c}^{2}$, and where $b=m_{e} V_{\perp} \omega_{c}$ for a motional Stark field and $b=e F$ for a dc external Stark field. In the above potential the electric field is perpendicular to the magnetic field. Note on the right how the linear Stark term results in a shift of the electronic distribution off-center whereas the constant term renormalizes the system's energy. When the electric field becomes increasingly large, a potential "bow" develops in the outer region. The motion of an excited electron in such a double valley potential has not been studied much. ${ }^{(16)}$

We now turn our attention to the remarkable occurrence of either $\frac{1}{2} \hbar \omega_{c}$ or $\frac{3}{2} \hbar \omega_{c}$ spacings as a result of competing effects of different forces. We discuss the conditions under which one observes either one of the two spacings in terms of the atomic mass, the quantum defect, and the external magnetic and electric fields. ${ }^{(120)}$ There are two relevant quantum numbers which govern the transition between these two modes. The first is the effective quantum number for the quasi-Landau resonance around the threshold where the Coulomb field is comparable to the diamagnetic potential. As given for instance by WKB [cf. Eq. (18)] this is

$$
n_{L}=(41.4) B^{-1 / 3}
$$

where $B$ is in units of $5 \times 10^{4} \mathrm{G}$. This number represents the onset where " $n$ and $l$ mixing" is complete, giving rise to the $\frac{3}{2} \hbar \omega_{c}$ spacing. The quasiLandau resonances appear for final states having $l_{f}-m_{f}=$ even. ${ }^{(11,14)}$ Since we are in the strong magnetic field domain, the linear Zeeman effect reduces to the Paschen-Back limit where orbit and spin become uncoupled. We can thus deal with orbital motion only. Atoms with isotropic initial states, i.e., $l_{i}=0$, would show the resonances only in $\sigma$-polarization, one-photon transitions. If the lower state is anisotropic, i.e., $l_{i} \geqslant 1$, these resonances can be seen in both $\sigma$ and $\pi$ polarizations. This is because a one-photon transition from the lower state with orbital angular momentum $l$ leads to final states with $l^{\prime}=l \pm 1$, whereas $\sigma$ and $\pi$ polarizations induce transitions in the magnetic field to upper states with $m^{\prime}=m$ and $m^{\prime}=m \pm 1$, respectively. Thus, both polarizations, $\sigma$ and $\pi$ can lead to final states satisfying the $l^{\prime}-m^{\prime}=$ even rule.

On the other hand, the presence of a transverse electric field (due to either an external dc field or a motional Stark field) mixes both $m$ and $l$. However, it still confines the electron's motion in the same plane perpendicular to the magnetic field. If this " $m$-mixing" is not appreciable by the time the above $n, l$-mixing is complete, one will see $\mathrm{a} \frac{3}{2} \hbar \omega_{c}$ mode. If, instead, this $m$-mixing is complete by that stage (i.e., before $n_{L}$ is reached), one should observe the $\frac{1}{2} \hbar \omega_{c}$ mode. The index of Stark $m$-mixing is the relative probability of transitions to the $m$-forbidden components. Replacing the motional Stark potential in Eq. (81) by $e \mathbf{r} \cdot \mathbf{F}$ for an external Stark field, 
the value $n_{s}$ at which $m$-mixing is complete is defined as the point where the above ratio equals unity ${ }^{(120)}$

$$
R=\left[\frac{e F n_{s}^{2} a_{0}\left(1+D_{n}\right)}{e B \hbar / 2 m_{e} c+\operatorname{Ry}\left(2 / n_{s}^{3}\right) \Delta \mu}\right]^{2}=1
$$

Here $\Delta \mu$ is the difference in quantum defect between the $p$ and $f$ orbitals, and $D_{n}$ is the quantum defect correction discussed in Section 4.

We now compare these two indices, $n_{L}$ and $n_{S}$. Setting them equal will give the minimum $F$ field necessary for any $B$ to go from the $3 / 2$ to the $1 / 2$ mode with all lines having equal intensity. To get a compact expression for this, replace $n_{S}$ in the denominator of Eq. (85) by $n_{L}$ from (84) to obtain

$$
n_{S}=\left[\frac{a \Delta \mu+b}{0.529 \times 10^{-5}} \frac{B}{F}\right]^{1 / 2}
$$

where $F$ is in $\mathrm{V} / \mathrm{cm}, a=0.385$, and $b=0.29$ for hydrogenlike atoms. Equating Eqs. (86) and (84) we have the condition for the critical electric field, $F_{c}$, below which one observes the $3 / 2$ and above which the $1 / 2$ mode.

For $\mathrm{Li}, \Delta \mu=0.05, F_{\mathrm{c}}=34 B^{5 / 3}$. For $\mathrm{Ba}, \Delta \mu=0.15, F_{c}=38 B^{5 / 3}$, where $B$ is in $5 \times 10^{4} \mathrm{G}$ and $F$ in $\mathrm{V} / \mathrm{cm}$. For experiments performed in a cell, the motional Stark field $F_{\mathrm{th}}$ is always present. Assuming a temperature $T \approx 10^{3 \circ} \mathrm{K}$ for both $\mathrm{Li}$ and $\mathrm{Ba}$, the induced Stark field for a $B$ field in units of $5 \times 10^{4} \mathrm{G}$ is $F_{\mathrm{th}}=70 \mathrm{~B}$ for $\mathrm{Li}$ and $F_{\mathrm{th}}=18 \mathrm{~B}$ for Ba. For the case of $\mathrm{Li}$, it is noted that the motional Stark field is more than enough to give the full $m$-mixing and, thereby, the $\frac{1}{2} \hbar \omega_{c}$ mode. It is not until one reaches $B>17 \times 10^{7} \mathrm{G}$ that one can have $F_{c}>F_{\text {th }}$ and thus observe $\frac{3}{2} \hbar \omega_{c}$ in $\mathrm{Li}$. On the other hand, in $\mathrm{Ba}$ at $5 \times 10^{4} \mathrm{G} F_{c}>F_{\text {th }}$ so that the $m$-mixing is

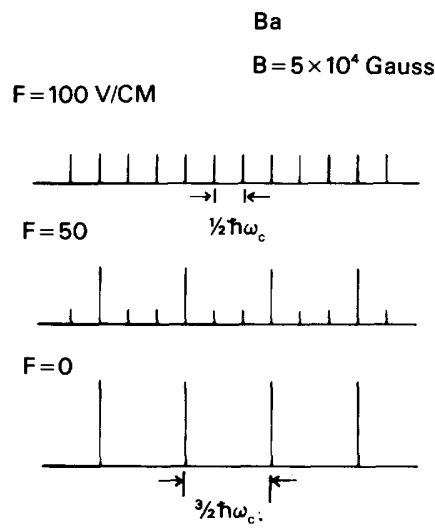

Figure 24. Schematic spectrum of Ba near threshold in crossed electric and magnetic fields. 
not appreciable and one observes the $\frac{3}{2} \hbar \omega_{c}$ mode. In fact, this is true for all fields larger than $7.5 \times 10^{3} \mathrm{G}$. Only at small fields is $F_{\mathrm{th}}>F_{c}$. One dramatic effect of this study of competition of forces is that one can change the spacing from $\frac{3}{2} \hbar \omega_{c}$ into $\frac{1}{2} \hbar \omega_{c}$ by applying an external transverse electric field. For example, for $\mathrm{Ba}$ in the vapor cell at $B=5 \times 10^{4} \mathrm{G}$, one will observe the $\frac{3}{2} \hbar \omega_{c}$ mode up to an $F_{\text {ext }}$ value of about $50 \mathrm{~V} / \mathrm{cm}$. After that one should see the $\frac{1}{2} \hbar \omega_{c}$ mode and the $\frac{1}{2} \hbar \omega_{c}$ resonances become uniform in intensity for $F_{\text {ext }}>100 \mathrm{~V} / \mathrm{cm}$. Figure 24 shows the schematic spectra of this phenomenon for $\mathrm{Ba}$. The ratio in Eq. (85) measures the ratio of intensity of peak height of the spectral resonance with its neighbors in Figure 24. Other theoretical work on atoms in joint external magnetic and electric fields has been reviewed by Bayfield. ${ }^{(19)}$

\section{General Properties of Atoms in Magnetic Fields of Astrophysical Strength}

The possibility of magnetic fields of order $10^{7} \mathrm{G}$ on white dwarf stars and of $10^{12} \mathrm{G}$ on neutron stars has stimulated theoretical interest in the nature of atoms and of atomic processes under such high-field conditions. Magnetic fields of $10^{9} \mathrm{G}$ or greater are capable of significantly compressing even the motion of ground-state electrons in the direction perpendicular to the magnetic field. The theory for the lowest levels of atomic hydrogen under strong field conditions has been discussed in Section 2.2.3 above. Atoms heavier than hydrogen have not been as well studied in the highmagnetic-field domain. Some of their general properties are, however, qualitatively understood. Furthermore, the presence of a high magnetic field makes possible new states of matter and also introduces characteristic resonance behavior in atomic scattering processes. We discuss these features briefly below. Note that the astrophysical applications of the theory have been reviewed by Garstang, ${ }^{(17)}$ and thus we do not discuss them here.

\subsection{Atomic Shell Structure}

For magnetic fields larger than $10^{9} \mathrm{G}$ the magnetic field confines electronic motion in the direction perpendicular to the field to within a cylinder of radius smaller than $a_{0}$, the Bohr radius. In the axial direction the electron is still primarily influenced by the attractive Coulomb field. In fact, for the lowest state of motion in $z$ the electron's wave function is of even parity and hence has a large amplitude near the nucleus. Physically, the binding energy of this lowest state increases with increasing magnetic field strength. Higher states of motion in the $z$-direction are not nearly so tightly bound. 
It is instructive to consider the limit of infinitely strong magnetic field strength. ${ }^{(77,128,129)}$ This case will have energy levels similar to those for free electrons in a uniform magnetic field ${ }^{(128)}$

$$
E=\hbar\left(\frac{e B}{\mu c}\right)\left(n_{\rho}+\frac{1}{2}|m|-\frac{1}{2} m+\frac{1}{2}\right)+E_{z}
$$

Here $n_{\rho}$ is the quantum number for motion in the $\rho$ direction, $m$ is the magnetic quantum number, and $E_{z}$ is the energy for motion along the $z$ axis. One sees that for a neutral atom of atomic number $Z$, the lowest energy state would be that in which all electrons were in the lowest Landau level $n_{\rho}=0$ with magnetic quantum numbers $m=0,1, \ldots, Z-1$, since each Landau level is infinitely degenerate. All electrons would also be in the strongly bound ground state for motion along $z$ (i.e., the lowest bound energy for $E_{z}$ ) and all would have their spins antialigned with the magnetic field. For successively larger $m$ values, the mean radius $\rho$ of the electrons would become slightly larger. Thus the atomic shell structure would resemble a set of concentric cylinders of finite length, as shown in Figure 25.

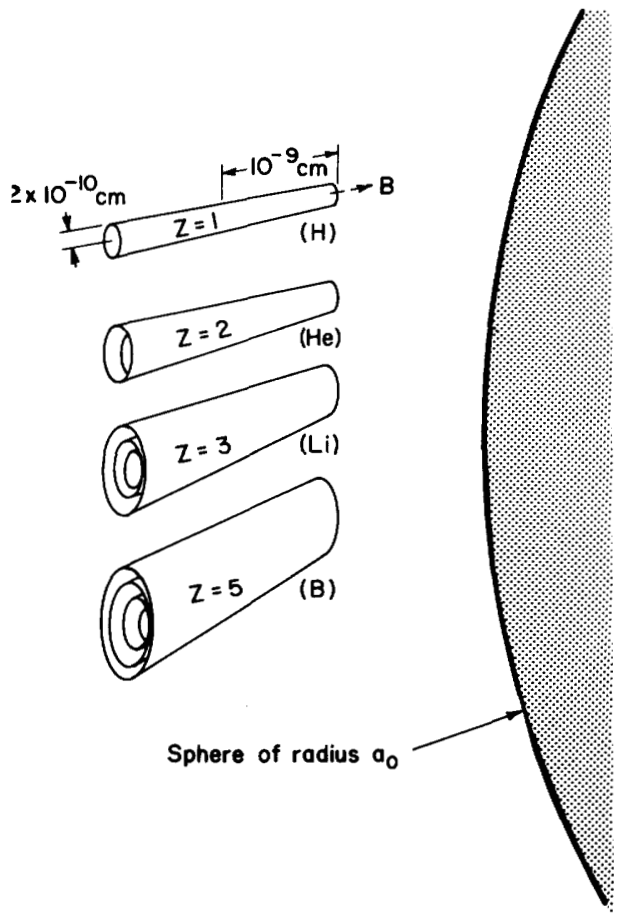

Figure 25. Shapes and sizes of light atoms in a $2 \times 10^{12} \mathrm{G}$ field. (From Ref. 129.) 
For any finite strength magnetic field, the atomic structure for a particular atom will lie between the extremes of normal atomic structure and the structure described above for an extremely large magnetic field. Because of the nonseparability of the combined Coulomb and magnetic potentials it is not possible to theoretically trace the development of a particular atomic level with increasing magnetic field, although several authors have made approximate attempts to do so. ${ }^{(63)}$ However, Cohen $e t$ al., ${ }^{(77)}$ Ruderman, ${ }^{(129)}$ and Rau et $a l .{ }^{(130)}$ have given detailed quantitative discussions of atomic structure in fields of the order of $10^{12} \mathrm{G}$.

\subsection{Magnetic-Field-Induced Binding}

The magnetic field confinement of electronic motion in the $\rho$ direction has important implications for the binding of the electron. Whereas attractive three-dimensional potentials do not necessarily have bound states, all attractive one-dimensional potentials have at least one bound state. Thus, for magnetic fields strong enough to confine electronic motion to one dimension (i.e., along the field) the electron will always have at least one bound state if the potential it moves in is at all attractive. This point was made by Avron et al. ${ }^{(131)}$ in a short report which states that $\mathrm{H}^{-}$can be shown to have an infinity of bound states in a large magnetic field and that $\mathrm{He}^{-}$has at least one bound state in a large magnetic field. Note that polarization effects of the outer electron on the neutral atom lead to an attractive potential between the two. Also, Larsen ${ }^{(132)}$ has performed variational calculations on $\mathrm{H}^{-}$in a magnetic field. He then used his calculations to interpret certain features in experimental magneto absorption data on CdS as due to photodetachment of negative donor ions.

Related to this work on negative ions are theoretical calculations of Ozaki and Tomishima on the $\mathrm{H}_{2}^{+}$molecule in a uniform magnetic field. ${ }^{(133)}$ In the absence of a magnetic field, the $1 \pi g$ state of $\mathrm{H}_{2}^{+}$is an antibonding state. For sufficiently strong magnetic fields, however, Ozaki and Tomishima find that this state changes to a bonding state. Clearly, then, the phenomenon of magnetic-field-induced binding is quite a general one.

\subsection{Landau Level Resonances}

As discussed earlier in this article, the existence of resonances above the zero-field ionization limit in studies of atomic photoabsorption in the presence of laboratory-sized magnetic fields (i.e., $B \approx 10^{5} \mathrm{G}$ ) was a novelty. These resonances were explained theoretically as due to quasibound motion of the photoelectrons in the direction perpendicular to the magnetic field. Eventually, due to Coulomb interactions with the ionic core, the electron escapes from the ion along the magnetic field direction. 
For very high magnetic fields similar behavior is predicted theoretically for a number of ionization processes. Thus sharp resonances are predicted in atomic photoionization, ${ }^{(134)}$ in photodetachment of negative ions, ${ }^{(135)}$ and in electron scattering. ${ }^{(136,137)}$ Such resonance behavior is thus a very general feature of continuum electron motion in combined Coulomb and uniform magnetic fields.

\section{Acknowledgments}

We wish to thank Ugo Fano, David Harmin, A. R. P. Rau, and Ken Taylor for many stimulating discussions. Alan Edmonds, David Harmin, and Richard Pullen have kindly provided results prior to publication.

\section{References and Notes}

1. P. Zeeman, Phil. Mag. 5, 43 (1897).

2. J. Stark, Berl. Akad. Wiss. 40, 932 (1913).

3. F. Paschen and E. Back, Ann. Phys. (Leipzig) 39, 897 (1912).

4. J. H. Van Vleck, The Theory of Elastic and Magnetic Susceptibilities, Oxford University Press, Oxford (1932).

5. F. A. Jenkins and E. Segré, Phys. Rev. 55, 52 (1939); E. Segré, Nuovo Cimento 11, 304 (1934).

6. L. D. Landau, Z. Phys. 64, 629 (1930).

7. W. R. S. Garton and F. S. Tomkins, Astrophys. J. 158, 839 (1969).

8. R. J. Fonck, F. L. Roesler, D. H. Tracy, K. T. Lu, F. S. Tomkins, and W. R. S. Garton, Phys. Rev. Lett. 39, 1513 (1977).

9. P. Jacquinot, S. Liberman, and J. Pinard, Centre National de la Recherche Scientifique, Laboratoire Aimé Cotton, Orsay, France, report No. 273 (1977); S. Feneuille, S. Liberman, J. Pinard, and P. Jacquinot, C.R. Acad. Sci. Paris, Ser. B 284, 291 (1977); J. C. Gay, D. Delande, and F. Biraben, J. Phys. B 13, L729 (1980).

10. M. G. Littman, M. L. Zimmerman, and D. Kleppner, Phys. Rev. Lett. 37, 46 (1976).

11. K. T. Lu, F. S. Tomkins, and W. R. S. Garton, Proc. R. Soc. London, Ser. A 364, 421 (1978).

12. R. R. Freeman, N. P. Economou, G. C. Bjorklund, and K. T. Lu, Phys. Rev. Lett. 41, 1463 (1978).

13. K. T. Lu, F. S. Tomkins, H. M. Crosswhite, and H. Crosswhite, Phys. Rev. Lett. 41, 1034 (1978).

14. U. Fano, Colloq. Int. C.N.R.S. 273, 127 (1977).

15. H. Crosswhite, U. Fano, K. T. Lu, and A. R. P. Rau, Phys. Rev. Lett. 42, 963 (1979).

16. A. R. P. Rau, J. Phys. B 12, L193 (1979); and Comments At. Mol. Phys. 10, 19 (1980).

17. R. H. Garstang, Rep. Prog. Phys. 40, 105 (1977).

18. K. J. Kollath and M. C. Standage, Progress in Atomic Spectroscopy, Part B, p. 955, Eds. W. Hanle and H. Kleinpoppen, Plenum Press, New York (1978).

19. J. E. Bayfield, Phys. Rep. 51, 319 (1979).

20. D. Kleppner, Les Houches Summer School, Session 28, Eds. J. C. Adam and R. Ballian, Gordon Breach, New York (1981). 
21. F. S. Ham, Solid State Phys. 1, 127 (1955).

22. M. J. Seaton, Proc. Phys. Soc. 88, 801 (1966); J. Phys. B: Atom. Molec. Phys. 11, 4067 (1978).

23. U. Fano, Phys. Rev. A 2, 353 (1970); J. Opt. Soc. Am. 65, 979 (1975); Phys. Rev. A 15, 817 (1977).

24. K. T. Lu and U. Fano, Phys. Rev. A 2, 81 (1970); K. T. Lu, Phys. Rev. A 4, 579 (1971); C. M. Lee and K. T. Lu, Phys. Rev. A 8, 1241 (1973).

25. W. E. Lamb, Phys. Rev. 85, 259 (1952).

26. H. A. Bethe and E. E. Salpeter, Quantum Mechanics of One-and Two-Electron Atoms, Springer-Verlag, Berlin (1957).

27. B. P. Carter, J. Math. Phys. 10, 788 (1969).

28. H. Grotch and R. A. Hegstrom, Phys. Rev. A 4, 59 (1971).

29. M. Sh. Ryvkin, Dokl. Akad. Nauk SSSR 221, 67 (1975). [Eng. Transl.: Sov. Phys. Dokl. 20, 192 (1975).]

30. J. E. Avron, I. W. Herbst, and B. Simon, Ann. Phys. (N.Y.) 114, 431 (1978).

31. R. F. O'Connell, Phys. Lett. 70A, 389 (1979).

32. G. Wunner and H. Herold, Astrophys. Space Sci. 63, 503 (1979).

33. G. Wunner, H. Ruder, and H. Herold, Phys. Lett. 79A, 159 (1980).

34. H. Herold, H. Ruder, and G. Wunner, J. Phys. B 14, 751 (1981).

35. A. R. Edmonds, J. Phys. (Paris) 31, C4, 71 (1970).

36. A. F. Starace, J. Phys. B 6, 585-590 (1973).

37. R. Gajewski, Physica 47, 575 (1970).

38. W. R. S. Garton, F. S. Tomkins, and H. M. Crosswhite, Proc. R. Soc. London, Ser. A 373, 189 (1980).

39. A. R. Edmonds and R. A. Pullen, Imperial College Preprints ICTP/79-80/28, 29, 30; and R. A. Pullen, thesis, Imperial College, London (1981).

40. V. I. Arnold and A. Avez, Ergodic Problems of Classical Mechanics, Benjamin, New York (1968). See also D. Delande and J. C. Gay, Phys. Lett. 82A, 393 (1981).

41. I. Percival, J. Phys. B 6, L229 (1973).

42. C. J. Goebel and T. W. Kirkman (to be published).

43. C. Lanczos, The Variational Principles of Mechanics, University of Toronto Press, Toronto (1949).

44. U. Fano, Phys. Rev. A 22, 2660 (1980).

45. U. Fano, J. Phys. B 13, L519 (1980).

46. G. H. Wannier, Phys. Rev. 90, 817 (1953).

47. C. W. Clark and K. T. Taylor, 1st Europe. Conf. Atomic Phys. Abstracts, p. 144 (1981); J. Phys. B 15, 1175 (1982).

48. J. Killingbeck, J. Phys. B 12, 25 (1979).

49. J. E. Avron, B. G. Adams, J. Cizek, M. Clay, M. L. Glasser, P. Otto, J. Paldus, and E. Vrscay, Phys. Rev. Lett. 43, 691 (1979).

50. D. R. Bates and A. Damgaard, Phil. Trans. R. Soc. London, Ser. A 242, 101 (1949).

51. J. Picart, A. R. Edmonds, and N. Tran Minh, J. Phys. B 11, L651 (1978).

52. G. Risberg, Ark. Fys. 28, 381 (1965).

53. C. M. Brown, R. H. Naber, S. G. Tilford, and M. L. Ginter, Appl. Opt. 12, 1858 (1973).

54. C. W. Clark, Phys. Rev. A 24, 605 (1981).

55. Ref. 26, p. 29.

56. G. Szegö, Orthogonal Polynomials, American Mathematical Society, Providence, Rhode Island (1939).

57. The substance of this paragraph has been largely developed from remarks by U. Fano (private communication).

58. M. L. Zimmerman, M. M. Kash, and D. Kleppner, Phys. Rev. Lett. 45, 1092 (1980). 
59. C. W. Clark and K. T. Taylor, J. Phys. B 13, L737 (1980).

60. C. W. Clark and K. T. Taylor, Nature 292, 437 (1981).

61. A. R. Edmonds, J. Phys. B 6, 1603 (1973).

62. C. B. Crawford, Comm. ACM 16, 41 (1973).

63. H. Hasegawa, in Physics of Solids in Intense Magnetic Fields, Ed. E. D. Haidemenakis, Plenum Press, New York (1969), Chap. 10.

64. A. F. Starace and G. L. Webster, Phys. Rev. A 19, 1629 (1979).

65. G. L. Webster, Doctoral Dissertation, The University of Nebraska-Lincoln (1981).

66. H. Hellmann, Einführung in die Quantenchemie, Deuticke, Leipzig, Germany (1937); R. P. Feynmann, Phys. Rev. 56, 340 (1939).

67. See Ref. 65, Tables III-V for the best variational results in each instance.

68. A. Baldereschi and F. Bassani, in Proceedings of the 10th International Conference on the Physics of Semiconductors, Cambridge, Massachusetts, AEC Oak Ridge CONF700801, Eds. S. P. Keller, J. C. Hensel, and F. Stern, U.S. AEC., Washington, D.C. (1970), pp. 191-196.

69. L. I. Schiff and H. Snyder, Phys. Rev. 55, 59 (1939).

70. R. J. Elliott and R. Loudon, J. Phys. Chem. Solids 15, 196 (1960).

71. H. Hasegawa and R. E. Howard, J. Phys. Chem. Solids 21, 179 (1961).

72. A. G. Zhilich and B. S. Monozon, Fiz. Tverd. Tela (Leningrad) 8, 3559 (1966) [Sov. Phys. Solid State 8, 2846 (1967)].

73. V. Canuto and D. C. Kelly, Astrophys. Space Sci. 17, 277 (1972).

74. Y. Yafet, R. W. Keyes, and E. N. Adams, J. Phys. Chem. Solids 1, 137 (1956).

75. D. M. Larsen, J. Phys. Chem. Solids 29, 271 (1968).

76. E. P. Polkatilov and M. M. Rusanov, Fiz. Tverd. Tela (Leningrad) 10, 3117 (1968) [Sov. Phys. Solid State 10, 2458 (1969)].

77. R. Cohen, J. Lodenquai, and M. Ruderman, Phys. Rev. Lett. 25, 467 (1970).

78. J. Callaway, Phys. Lett. A 40, 331 (1972).

79. A. K. Rajagopal, G. Chanmugan, R. F. O'Connell, and G. L. Surmelian, Astrophys. J. 177, 713 (1972).

80. (a) E. R. Smith, R. J. W. Henry, G. L. Surmelian, R. F. O'Connell, and A. K. Rajagopal, Phys. Rev. D 6, 3700 (1972); (b) G. L. Surmelian and R. F. O'Connell, Astrophys. J. 190, 741 (1974).

81. L. W. Wilson, Astrophys. J. 188, 349 (1974).

82. H. S. Brandi, Phys. Rev. A 11, 1835 (1975).

83. A. R. P. Rau and L. Spruch, Astrophys. J. 207, 671 (1976).

84. R. R. dos Santos and H. S. Brandi, Phys. Rev. A 13, 1970 (1976).

85. R. K. Bhaduri, Y. Nogami, and C. S. Warke, Astrophys. J. 217, 324 (1977).

86. J. M. Wadehra, Astrophys. J. 226, 372 (1978).

87. D. J. Hylton and A. R. P. Rau, Phys. Rev. A 22, 321 (1980).

88. D. Cabib, E. Fabri, and G. Fiorio, Solid State Commun. 9, 1517 (1971).

89. H. C. Praddaude, Phys. Rev. A 6, 1321 (1972).

90. R. H. Garstang and S. B. Kemic, Astrophys. Space Sci. 31, 103 (1974).

91. W. Ekardt, Solid State Commun. 16, 233 (1975).

92. A. Galindo and P. Pascual, Nuovo Cimento B 34, 155 (1976).

93. E. A. Hylleraas and B. Undheim, Z. Phys. 65, 759 (1930); E. C. Kemble, The Fundamental Principles of Quantum Mechanics, McGraw-Hill, New York (1937), Section $51 \mathrm{C}$.

94. J. E. Avron, I. W. Herbst, and B. Simon, Phys. Lett. 62A, 214 (1977); Phys. Rev. A 20, 2287 (1980).

95. J. E. Avron, Ann. Phys. (N.Y.) 131, 73 (1981).

96. L. Haines and D. Roberts, Am. J. Phys. 37, 1145 (1969). 
97. L. D. Landau and E. M. Lifshitz, Quantum Mechanics (Non-Relativistic Theory), 3rd ed., Pergamon, Oxford (1976).

98. C. Lanczos, Z. Phys. 65, 431 (1930).

99. E. Segré and G. C. Wick, Proc. R. Soc. Amsterdam 36, 534 (1933).

100. R. E. Langer, Phys. Rev. 51, 669 (1937).

101. M. H. Rice and R. H. Good, Jr., J. Opt. Soc. Am. 52, 239 (1962).

102. D. S. Bailey, J. R. Hiskes, and A. C. Riviere, Nucl. Fus. 5, 41 (1965).

103. R. J. Damburg and V. V. Kolosov, J. Phys. B 9, 3149 (1976); Phys. Lett. 61A, 233 (1977); J. Phys. B 11, 1921 (1978).

104. H. M. Silverstone, Phys. Rev. A 18, 1853 (1978); and P. M. Koch, Phys. Rev. Lett. 41, 99 (1978).

105. B. E. Cole, J. W. Cooper, and E. B. Saloman, Phys. Rev. Lett. 45, 887 (1980); and J. W. Cooper and E. B. Saloman, Phys. Rev. A 26, 1452 (1982).

106. W. Sandner, K. Safinya, and T. Gallagher, Phys. Rev. A 23, 2488 (1981).

107. T. S. Luk, L. DiMauro, T. Bergeman, and H. Metcalf, Phys. Rev. Lett. 47, 83 (1981).

108. A. R. P. Rau and K. T. Lu, Phys. Rev. A 21, 1057 (1980); K. T. Lu, J. Opt. Soc. Am. 68, 1446 (1978).

109. E. Luc-Koenig and A. Bachelier, Phys. Rev. Lett. 43, 921 (1979); J. Phys. B 13, 1743 (1980); J. Phys. B 13, 1769 (1980).

110. D. F. Blossey, Phys. Rev. B 2, 3976 (1970).

111. V. D. Kondratovich and V. N. Ostrovskii, Sov. Phys. JETP 52, 198 (1980).

112. D. A. Harmin, Phys. Rev. A 24, 2491 (1981).

113. C. Bender and T. T. Wu, Phys. Rev. 184, 1231 (1969); Phys. Rev. Lett. 16, 461 (1971); Phys. Rev. D 7, 1620 (1973).

114. L. Benassi, V. Grecchi, E. Harrell, and B. Simon, Phys. Rev. Lett. 42, 704 (1979).

115. H. J. Silverstone, B. G. Adams, Jiri Cizek, and P. Otto, Phys. Rev. Lett. 43, 1498 (1979).

116. D. Park, Z. Phys. 159, 155 (1960).

117. A. R. P. Rau, unpublished.

118. D. A. Harmin, Phys. Rev. A 26, 2656 (1982).

119. U. Fano, Phys. Rev. A 24, 619 (1981); and D. A. Harmin, Phys. Rev. Lett. 49, 128 (1982).

120. K. T. Lu and A. R. P. Rau, unpublished; and 7th International Conference on Atomic Physics Abstracts, p. 21, MIT, 1980; and J. C. Gay, L. R. Pendrill, and B. Cagnac, Phys. Lett. 72A, 315 (1979).

121. J. A. Armstrong, J. J. Wynne, and P. Esherick, J. Opt. Soc. Am. 69, 211 (1979).

122. M. Aymar, P. Camus, M. Dieulin, and C. Marillon, Phys. Rev. A 18, 2173 (1978); M. Aymar and O. Robaux, J. Phys. B: Atom. Molec. Phys. 12, 531 (1979).

123. C. H. Greene, Phys. Rev. A 23, 661 (1981).

124. K. T. Lu, Proc. R. Soc. London, Ser. A 353, 431 (1977).

125. J. J. Wynne, J. A. Armstrong, and P. Esherick, Phys. Rev. Lett. 39, 1520 (1977).

126. J. Geiger, J. Phys. B 12, 2277 (1979).

127. M. Rosenbluh, T. A. Miller, D. M. Larson, and B. Lax, Phys. Rev. Lett. 39, 874 (1977);

M. Rosenbluh, R. Panock, B. Lax, and T. A. Miller, Phys. Rev. A 18, 1103 (1978).

128. L. D. Landau and E. M. Lifshitz, Quantum Mechanics, 2nd Ed., Addison-Wesley, Reading, Massachusetts (1965), Section 111.

129. M. Ruderman, in Physics of Dense Matter (I.A.U. Symposium No. 53), Ed. by C. J. Hansen, D. Reidel, Boston (1974), pp. 117-131.

130. A. R. P. Rau, R. O. Mueller, and L. Spruch, Phys. Rev. A 11, 1865 (1975).

131. J. Avron, I. Herbst, and B. Simon, Phys. Rev. Lett. 39, 1068 (1977).

132. D. M. Larsen, Phys. Rev. Lett. 42, 742 (1979).

133. J. Ozaki and Y. Tomishima, J. Phys. Soc. Jpn. 49, 1497 (1980).

134. S. M. Kara and M. R. C. McDowell, J. Phys. B 14, 1719 (1981). 
135. W. A. M. Blumberg, W. M. Itano, and D. J. Larsen, Phys. Rev. A 19, 139 (1979).

136. K. Onda, J. Phys. Soc. Jpn. 45, 216 (1978).

137. G. Ferrante, S. Nuzzo, M. Zarcone, and S. Bivona, J. Phys. B 13, 731 (1980).

138. M. Robnik, J. Phys. A 14, 3198 (1981).

139. E. A. Soloviev, Pis'ma Zh. Eksp. Teor. Fiz. 34, 278 (1981) [JETP Lett. 34, 265 (1981)]; Zh-Eksp. Teor. Fiz. 82, 1762 (1982).

140. D. R. Herrick, Phys. Rev. A 26, 323 (1982).

141. J. J. Labarthe, J. Phys. B 14, L467 (1981). 